\title{
Role of a putative cyclic di-GMP forming locus MSMEG_2196 \\ in
}

\section{Mycobacterium smegmatis}

By

Janet Youkhana

\begin{abstract}
A thesis
Submitted to the Victoria University of Wellington

In fulfilment of the requirements for the degree of

Masters in Biomedical Science
\end{abstract}

Victoria University of Wellington

2008 


\section{Abstract}

Cyclic di-guanosine-monophosphate (c-di-GMP) has been recognized as a second messenger in bacteria controlling multiple cellular processes such as biofilm formation, motility, and virulence. Proteins containing GGDEF and EAL domains are engaged in the synthesis and degradation, respectively, of cyclic di-GMP. Some bacteria contain multiple proteins with GGDEF and EAL domains. The genome of Mycobacterium tuberculosis encodes only one protein (Rv1354c) which contains a GGDEF domain. This protein also contains a tandem EAL. The function of this protein in mycobacteria has not yet been determined.

In this study, the orthologue of Rv1354c was investigated in Mycobacterium smegmatis (MSMEG_2196). The expression of MSMEG_2196 in M. smegmatis was altered by constructing sense and antisense expressing strains. The effect of the altered expression of MSMEG_2196 on M. smegmatis was tested under carbon, oxygen, phosphorous, and nitrogen limited growth conditions. There was no significant effect on growth in either the antisense or sense expressing strains grown under nutrient-rich, or carbon-, or oxygen-, or phosphorous limitation conditions. However, a growth effect was observed in the antisense expressing strain when grown under nitrogen-limited conditions. In particular, at mid stationary-phase $(1,800 \mathrm{~min})$ the MSMEG_2196 antisense strain had an $\mathrm{OD}_{600}$ value of 0.60 , compared to that of the control M. smegmatis/pMind strain $\left(\mathrm{OD}_{600}\right.$ value of 1.09). These results were further confirmed by the low colony forming units measures observed in MSMEG_2196 antisense strain. Proteomic analysis was carried out on the MSMEG_2196 antisesne expressing strain grown in the nitrogen-limited condition. Proteins that were differentially expressed were identified by mass spectrometry. A number of the proteins that were down-regulated in the antisense expressing strain are important in the survival of the bacteria under nitrogen-limited conditions.

This study indicates a role for MSMEG_2196 in growth or survival of mycobacteria under nitrogen-limitations. 


\section{Chapter 1: Introduction}

\section{Acknowledgments}

First and foremost, I would like to thank my supervisor Dr. Ronan O'Toole for all his support, advice, and encouragements, without him this thesis will not be possible. Thank you to Dr. Des Collin and Pam Kawakami from AgResearch (Wallaceville, New Zealand) who have helped me with the work of this thesis and who gave me feedback, without them, the work based on $M$. bovis mutagenesis would have been impossible. Thanks to Dr. Bill Jordan who opened the door of his laboratory, and gave me the opportunity to carry out the proteomic work of this thesis, and a huge thank you go out to Jonathan Dunne who have guided me through the techniques of proteomics, and who also gave me feedback on the writing of my thesis. Thanks to my fellow postgraduate students from Microbiology laboratory who have made working in laboratory fun and enjoyable, thanks for their support. I would also like to thank the family of Dario Vazques for their support while being away from home, and special thanks to Dario Vazques who have supported me and encouraged me during the writing of my thesis. Finally, I would like to thank my family, especially my parents, for their continued support. Without them, I would not have been given all the opportunities I had and have.

Janet Youkhana 


\section{Table of Contents}

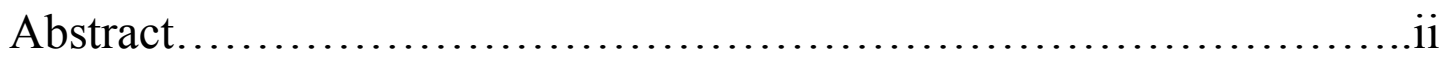

Acknowledgments................................................ii

Table of contents.................................................

List of Figures.................................................

List of Tables.....................................................

List of abbreviations.............................................

\section{Chapter 1: Introduction}

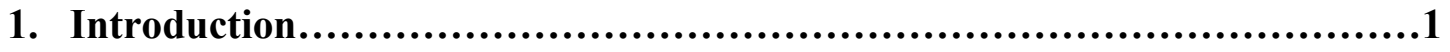

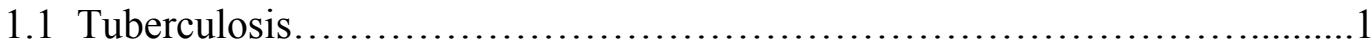

1.2 Finding new drug targets to treat TB.................................

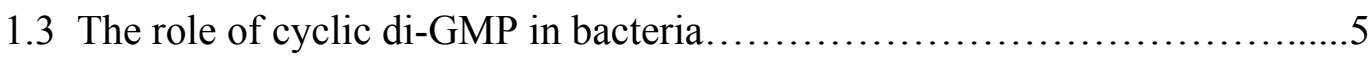

1.4 The synthesis and degradations of cyclic di-GMP in bacteria.................

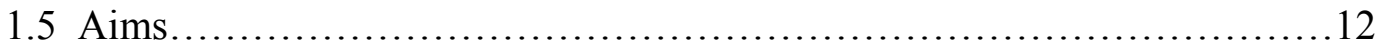

1.5.1 Determine whether a cyclic di-GMP enzyme is encoded by M. tuberculosis genome..........................................................

1.5.2 Construction of sense and antisense c-di-GMP strains in M. smegmatis.......12

1.5.3 Investigate the effect of the changed expression of c-di-GMP sense and antisense on the physiology of $M$. smegmatis........................13

1.5.4 Proteomic analysis of MSMEG_2196 in HdeB growth limited media.........13

1.5.5 Mutation of c-di-GMP in M. bovis and M. smegmatis, respectively...........13

\section{Chapter 2: Materials and Methods}

2. Materials and methods....................................................15

2.1 Bacterial strains, plasmids and culture conditions........................15

2.2 Bioinformatics of c-di-GMP in the genome of mycobacteria.................16

2.3 Altered expression of MSMEG_2196.................................17

2.3.1 Preparation of genomic DNA.......................................... 17

2.3.2 Preparation of Mycobacterium smegmatis $\mathrm{mc}^{2} 155$ electroporation-competent

cells.................................................................. 18

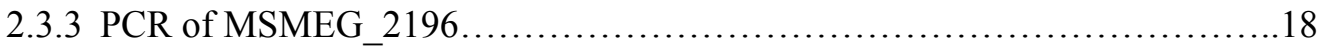

2.3.4 Ligation of MSMEG_2196 into pCR2.1 cloning vector..................20 
2.3.5 Heat shock transformation of MSMEG_2196-pCR2.1 fragment into E.coli

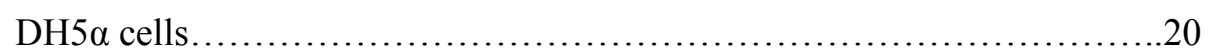

2.3.6 Midipreparaion, purification, and stock culture of MSMEG_2196-pCR2.1 plasmid

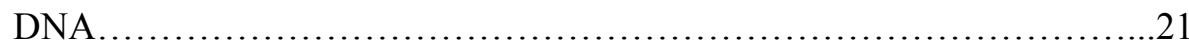

2.3.7 Plasmid digestion and gel purification of MSMEG_2196-pCR2.1............21

2.3.8 Sequencing of MSMEG_2196-pCR2.1 fragment..........................22

2.3.9 Ligation of MSMEG_2196 into the pMind expression plasmid..............22

2.3.10 Insert and orientation check of MSMEG_2196 into pMind plasmid.........23

2.3.11 Elecroporation of MSMEG_2196-antisense/sense-pMind into $M$.

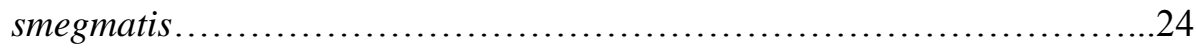

2.4 Investigating growth effects of MSMEG_2196-antisense/sense.............25

2.4.1 Growth curves..........................................................25

2.4.2 Determination of colony forming units.................................27

2.5 Proteomic analysis of Ms/p2196 antisense grown under nitrogen limited

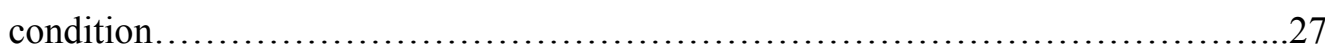

2.5.1 Protein of Ms/p2196 AS and Ms/pMind cells for one-dimension (1D) and two-dimension (2D) gel analysis.................................27

2.5.2 1D and 2D gel electrophoresis analysis of Ms/p2196 antisense expressing strain and Ms/pMind protein samples on $\mathrm{pH}$ 4-7 isoelectric focusing strips

2.5.3 1D and 2D gel electrophoresis analysis of Ms/p2196 antisense expressing strain and Ms/pMind protein samples on $\mathrm{pH}$ 6-11 isoelectric focusing strips. .30

2.5.4 Ms/p2196 antisense expressing strain and Ms/pMind 2D gel analysis.........31

2.5.5 Protein database search and protein identifications of Ms/p2196 antisense and

Ms/pMind peptide mass fingerprint .................................... 34

2.6 Site directed mutagenesis of Mb1389c and MSMEG_2196..................34

2.6.1 PCR of Mb1389c and MSMEG_2196 flanking region.......................34

2.6.2 Mb1389c overlap PCR.................................................. 35

2.6.3 MSMEG_2196 overlap PCR............................................. 36

2.6.4 Preparations of Mb1389c and MSMEG_2196 overlap PCR fragments for ligation

into pUHA778 knock-out vector......................................38

2.6.5 pUHA778 knock-out plasmid preparation.............................. 38

2.6.6 Ligation of Mb1389c- and MSMEG_2196-overlap fragments into pUHA778 and transformation into E. coli DH5 $\alpha$ cells 
2.6.7 Mb1389c-/MSMEG_2196-overlap insert check in pUHA778 vector and preparation for electroporation.

2.6.8 PCR and southern blot analysis for single homologues recombination of Mb1389c/pUHA778 in M. bovis..................................40

2.6.9 MSMEG_2196/pUHA778 electroporation into M. smegmatis................42

2.6.10 PCR and southern blot analysis of M. smegmatis/MSMEG_2196pUHA778 recombinants..........................................42

\section{Chapter 3: Results}

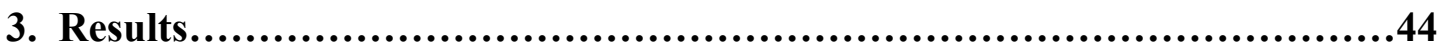

3.1 Bioinformatics of cyclic-di-GMP in mycobacteria........................44

3.1.1 Cyclic di-GMP protein encoded by the M. tuberculosis, M. bovis, and M. smegmatis genome.................................................44

3.1.2 Gene and protein sequences of Mb1389c and MSMEG_2196...............45

3.2 Altered expression of MSMEG_2196 in M. smegmatis.......................45

3.2.1 Amplification of MSMEG_2196....................................45

3.2.2 Cloning of the MSMEG_2196 gene into pCR2.1 vector...................47

3.2.3 Ligation of MSMEG_2196 into pMind and check for inserts................48

3.2.4 Checking the orientation of the MSMEG_2196 gene in pMind vector.........49

3.3 Growth assays of MSMEG_2196-pMind antisense/sense culture..............53

3.3.1 Nutrient rich growth assay of MSMEG_2196-pMind antisense/sense..........53

3.3.2 Carbon limited growth assay of MSMEG_2196-pMind antisense/sense........55

3.3.3 Oxygen limited growth assay of MSMEG_2196-pMind antisense/sense........57

3.3.4 Phosphorous limited growth assay of MSMEG_2196-pMind

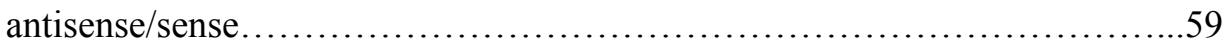

3.3.5 Nitrogen limited growth assay of MSMEG_2196-pMind antisense/sense.....62

3.3.6 CFU of Ms/pMind and Ms/p2196-AS in $\mathrm{N}^{-}$limited culture..................65

3.4 Proteomic of Ms/pMind and Ms/p2196 AS .............................67

3.4.1 Whole cell protein extraction from Ms/pMind and Ms/p2196 AS culture for

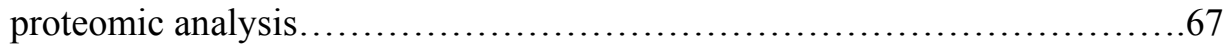

3.4.2 Analysis of differentially expressed proteins in Ms/pMind and Ms/p2196 AS strain grown in nitrogen-limited media...............................68

3.4.3 Proteins identified on 2D gel....................................... 72

3.5 Site directed mutagenesis of Mb1389c and MSMEG_2196................76

3.5.1 PCR of Mb1389c and MSMEG_2196 flanking region......................76

3.5.2 Overlap PCR of Mb1389c and MSMEG_2196.........................77 
3.5.3 Cloning of Mb1389c- and MSMEG_2196-overlap fragment into pGEM-T

Easy vector.

3.5.4 Digestion of Mb1389c- and MSMEG_2196-overlap fragment with Pac I enzyme for ligation into pUHA778 vector.

3.5.5 Ligation of Mb1389c and MSMEG_2196 Pac I digested fragments into pUHA778 plasmid................................................ 82

3.5.6 Generation of M. bovis-1389c mutants...................................83

3.5.7 Generation of M. smegmatis_2196 mutants.............................87

\section{Chapter 4: Discussion and future directions}

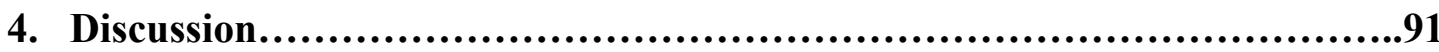

4.1 The effect of modification of MSMEG_2196 expression in M. smegmatis.....92

4.2 Proteins identified in Ms/p2196 antisense strain grown in nitrogen limited-

media............................................................... 94

4.2.1 Nitrogen metabolism in bacteria, and its possible role in MSMEG_2196 in

Ms/p2196 antisense culture..................................95

4.2.2 Other down-regulated proteins in Ms/p2196 antisense culture..........100

4.2.3 Up-regulated proteins in Ms/p2196 antisense culture..................102

4.3 Model of role of MSMEG_2196 in mycobacterial physiology and

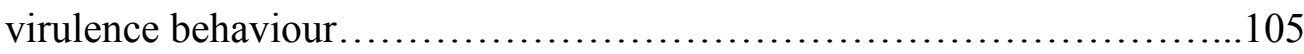

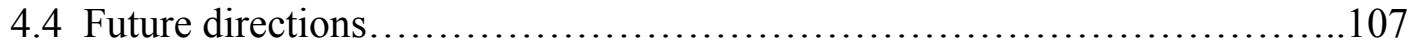

5 Appendices........................................................................110

5.1 Plasmids used..................................................... 110

5.1 .1 pUHA778................................................ 110

5.1 .2 pMind ............................................................ 111

5.2 Hartman-de Bonts (HdeB) minimal media.............................112

5.3 Preparation and electroporation of Mb1389c-pUHA778 into M. bovis Wag203

strain at AgResearch........................................... 114

5.3.1 Preparation of Mb1389c-pUHA778 deletion fragment

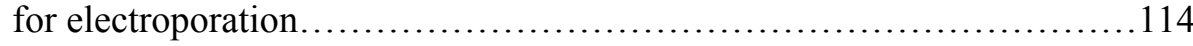

5.3.2 Electroporation of Mb1389c-pUHA778 into M. bovis Wag203...........114

5.4 Southern blot...................................................... 116

5.5 Gene alignments of Rv1354c against Mb1389c and MSMEG_2196.........120

5.5.1 Rv1354c sequence alignment against Mb1389c......................120

5.5.2 Rv1354c sequence alignment against MSMEG_2196.................123

$5.6 \mathrm{Mb1389c}$ and MSMEG_2196 gene and protein sequences.................126

5.6.1 Mb1389c gene sequence...................................... 126 
Chapter 1: Introduction

5.6.2 Mb1389c protein sequence.....................................126

5.6.3 MSMEG_2196 gene sequence....................................127

5.6.4 MSMEG_2196 protein sequence...................................127

6 References.........................................................................128 


\section{List of Figures}

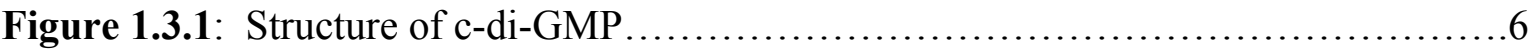

Figure 1.3.2: Synthesis and degradation of c-di-GMP $\ldots \ldots \ldots \ldots \ldots \ldots \ldots \ldots \ldots \ldots \ldots \ldots$

Figure 2.5.1: 2D gel spot intensity quantification using ImageJ software................32

Figure 2.5.2: Example of MALDI plate containing protein peptides of Ms/p2196 and external calibration sample. 33

Figure 3.1.1: Pfam domain search result of GGDEF domain in $M$. tuberculosis Rv1354c genome............................................................

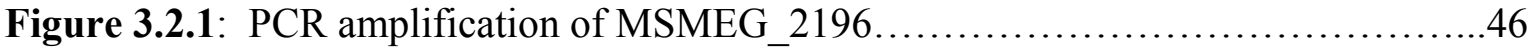

Figure 3.2.2: MSMEG_2196-pCR2.1 Pac I digested plasmid.........................47

Figure 3.2.3: $1 \%$ agarose gel of MSMEG_2196-pMind recombinant plasmids digested with Pac I enzyme.................................................. 48

Figure 3.2.4: Possible orientation of MSMEG_2196 in pMind $\ldots \ldots \ldots \ldots \ldots \ldots \ldots \ldots \ldots . . . \ldots 50$

Figure 3.2.5: $1 \%$ agarose gel of antisense and sense PCR of MSMEG_2196pMind .............................................................. 51

Figure 3.3.1: Growth curve of $M$. smegmatis WT, Ms/pMind, Ms/p2196 sense, and $\mathrm{Ms} / \mathrm{p} 2196$ antisense grown in LB broth rich media.......................54

Figure 3.3.2: Growth curve of $M$. smegmatis WT, Ms/pMind, Ms/p2196 sense, and Ms/p2196 antisense grown in HdeB $\mathrm{C}^{-}$limited media....................56

Figure 3.3.3: Growth curve of $M$. smegmatis WT, Ms/pMind, Ms/p2196 sense, and $\mathrm{Ms} / \mathrm{p} 2196$ antisense grown in $\mathrm{HdeB} \mathrm{O}^{-}$limited media..................58

Figure 3.3.4: Growth curve of $M$. smegmatis WT, Ms/pMind, Ms/p2196 sense, and $\mathrm{Ms} / \mathrm{p} 2196 a n t i s e n s e$ grown in $\mathrm{HdeB}^{-}$limited media

Figure 3.3.5: Growth curve of $M$. smegmatis WT, Ms/pMind, Ms/p2196 sense and $\mathrm{Ms} / \mathrm{p} 2196$

antisense grown in HdeB $\mathrm{N}^{-}$limited media. .64

Figure 3.4.1: SDS-PAGE of whole cell lysates of Ms/pMind and Ms/p2196 antisense grown in nitrogen limited condition. .68

Figure 3.4.2: $\mathrm{pH}$ 4-7 2D gel elecrophoresis of Ms/pMind (A) and Ms/p2196 antisense (B) .70

Figure 3.4.3: $\mathrm{pH}$ 6-11 2D gel electrophoresis of Ms/pMind (A) and Ms/p2196 antisense (B) 
Chapter 1: Introduction

Figure 3.4.4: Mascot search result of an identified protein.......................73

Figure 3.5.1: $1 \%$ agarose gel of Mb1389c flanking region PCR.....................76

Figure 3.5.2: $1 \%$ agarose gel of MSMEG_2196 flanking region PCR..................77

Figure 3.5.3: $1 \%$ agarose gel of Mb1389c overlap PCR...........................78

Figure 3.5.4: $1 \%$ agarose gel of MSMEG_2196 overlap PCR .....................78

Figure 3.5.5: $1 \%$ agarose gel of p1389c-overlap EcoR I digested fragment.............79

Figure 3.5.6: 1 \% agarose gel of p2196-overlap Pac I digested fragment................80

Figure 3.5.7: $1 \%$ agarose gel of Mb1389c-overlap Pac I digested fragment............81

Figure 3.5.8: $1 \%$ agarose gel of MSMEG_2196-overlap Pac I digested fragment........81

Figure 3.5.9: $1 \%$ agarose gel of Mb1389c-pUHA778 recombinant plasmids Pac I

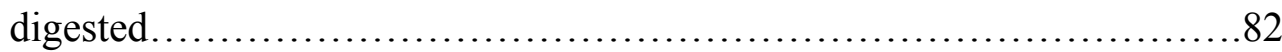

Figure 3.5.10: $1 \%$ agarose gel of MSMEG_2196-pUHA778 plasmid Pac I digested....83

Figure 3.5.11: $1 \%$ agarose gel of gDNA of $M$. bovis $\Delta 1389$ colonies..................85

Figure 3.5.12: $0.8 \%$ agarose gel of $M$. bovis $\Delta 1389$ gDNA digested with $B g l$ II enzyme.............................................. 85

Figure 3.5.13: The expected fragment size of first crossover of Mb1389c-pUHA778 in Southern blot................................................ 86

Figure 3.5.14: The expected fragment size of second crossover of Mb1389c-pUHA778 in Southern blot....................................................... 86

Figure 3.5.15: $0.8 \%$ agarose gel of gDNA of $M$. smegmatis $\Delta 2196$ digested with EcoN I enzyme....................................................... 88

Figure 3.5.16: The expected first cross-over fragment sizes of MSMEG_2196-pUHA778

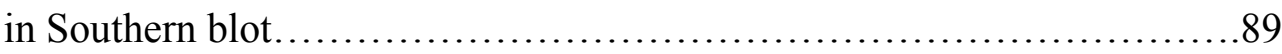

Figure 3.5.17: The expected fragment sizes of second cross-over of MSMEG_2196pUHA778 in Southern blot.................................... 89

Figure 4.2.1: Glutamine production in E. coli under nitrogen starvation.................97

Figure 4.2.2: Proposed c-di-GMP function in $M$. smegmatis.........................99 


\section{List of Tables}

Table 2.3.1: PCR conditions of MSMEG_2196................................ 19

Table 2.3.2: MSMEG_2196 antisense and sense construction primers..................19

Table 2.3.3: pMind TetRO primers for orientation check of MSMEG_2196 fragment in

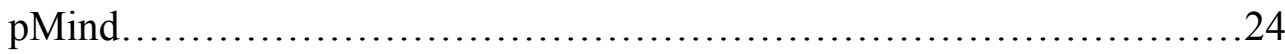

Table 2.6.1: Mb1389c and MSMEG_2196 flanking region and overlap PCR primers.....35

Table 2.6.2: $\mathrm{Mb} 1389 \mathrm{c}$ C1/D2 overlap PCR condition.............................. 36

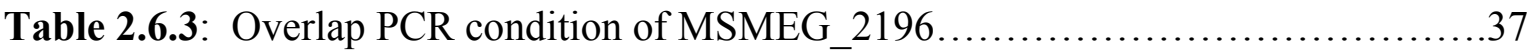

Table 2.6.4: $M$. bovis $1389 \Delta$ primers for analysis of $M$. bovis recombinants............41

Table 2.6.5: $\quad$ PCR primers used to check for MSMEG_2196 mutants in $M$. smegmatis.....................................................43

Table 3.1.1: Score and percentage identity of $M$. bovis and $M$. smegmatis c-di-GMP gene against $M$. tuberculosis Rv1354c...............................45

Table 3.2.1: Antisense and sense PCR results of MSMEG_2196-

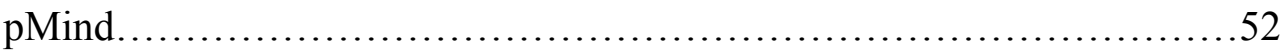

Table 3.3.1: Specific growth rates and doubling times for nutrient rich (LB broth)

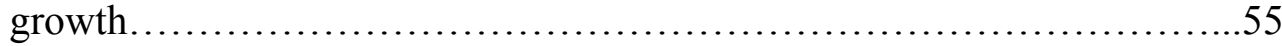

Table 3.3.2: Specific growth rates and doubling times for $\mathrm{C}^{-}$limited growth assay in HdeB media..................................................57

Table 3.3.3: Specific growth rates and doubling times for $\mathrm{O}^{-}$limited growth assay in HdeB media.

Table 3.3.4: Specific growth rates and doubling times for $\mathrm{P}^{-}$limited growth assay in HdeB media. .62

Table 3.3.5: Specific growth rates and doubling times for $\mathrm{N}^{-}$limited growth assay in HdeB media. .65

Table 3.3.6: CFU counts of Ms/pMind and Ms/p2196 AS grown in $\mathrm{N}^{-}$limited culture...66

Table 3.4.1: Proteins identified from 2D gel of Ms/pMind and Ms/p2196 AS...........74

Table 3.5.1: Summary of Mb1389c and MSMEG_2196 mutant construction work in $M$. bovis And M. smegmatis, respectively..............................90

Table 4.2.1: Down-regulated nitrogen regulatory proteins identified in 2D gel analysis of Ms/p2196 antisense expressing strain. 


\section{List of Abbreviations}

\begin{tabular}{|c|c|}
\hline bp & Base pair \\
\hline $\mathrm{CaCl}_{2}$ & Calcium Chloride \\
\hline CFU & Colony Forming Unit \\
\hline C-di-GMP & Cyclic di-Guanosine-Monophosphate \\
\hline DNA & Deoxyribonucleic acid \\
\hline DGC & Di-guanylate cyclase \\
\hline DMSO & Dimethyl sulfoxide \\
\hline DTT & Dithiothreitol \\
\hline XDR-TB & Extensively-drug-resistant tuberculosis \\
\hline hrs & Hours \\
\hline Hyg & Hygromycin \\
\hline IPTG & Isopropyl- $\beta$-thiogalacto pyranoside \\
\hline Kan & Kanamycin \\
\hline Kbp & Kilobase pair \\
\hline $\mathbf{K v}$ & Kilovolts \\
\hline $\mathrm{MgSO}_{4}$ & Magnesium sulphate \\
\hline $\boldsymbol{\mu} \mathbf{L}$ & Microliter \\
\hline $\mathbf{m L}$ & Milliliter \\
\hline $\mathbf{m M}$ & Millimolar \\
\hline $\operatorname{mins}$ & Minutes \\
\hline $\mathbf{M}$ & Molar \\
\hline MDR-TB & Multi-drug resistant tuberculosis \\
\hline ng & Nanogram \\
\hline OD $_{600}$ & Optical density of 600 \\
\hline PCR & Polymerase chain reaction \\
\hline PDEA & Phosphodiesterase A \\
\hline rpm & Revolutions per minute \\
\hline sec & Seconds \\
\hline SDS & Sodium dodecyl sulphate \\
\hline SDS-PAGE & Sodium dodecyl sulphate polyacrylamide \\
\hline SDW & Sterile distilled water \\
\hline WHO & Health \\
\hline
\end{tabular}




\section{Introduction}

\subsection{Tuberculosis}

Tuberculosis (TB), is caused by Mycobacterium tuberculosis, a slow growing (doubling time of $15-20 \mathrm{hrs}$ ), acid fast, rod shaped bacilli, $0.2-0.5 \mu \mathrm{m}$ in size. TB is an airborne communicable disease, transmitted from person to person via the respiratory system and can spread from the lungs to become a systemic infection if left untreated [147]. TB is still considered a "global epidemic infectious disease" and a major cause of death worldwide. In 2006, the World Health Organization (WHO) reported 9.2 million new cases worldwide and 1.7 million deaths caused by TB [147]. M. tuberculosis is a successful pathogen that evades the immune system of the host, establishing a chronic latent infection where it may silently persist inside a hostile environment such as the granuloma in the lung, specifically in pulmonary alveoli [141] (Reviewed in [140]). The granuloma is a nodule consisting of epithelioid macrophages, T and B lymphocytes, plasma cells, and fibroblasts [141] (Reviewed in [140]). Inside the granuloma, M. tuberculosis can escape chemotherapy and remain in the latent stage for many years [141 and 148] (Reviewed in [140] and [142]). This acts as a reservoir from which patients can develop active disease, especially patients who are immuno-compromised such as those with HIV or the elderly [141] (Reviewed in [140] and [142]). In addition, none of the conventional antituberculosis drugs are very effective against the "dormant bacilli". 


\section{Chapter 1: Introduction}

Some of the studies carried out on $M$. tuberculosis have focused on the environmental conditions of the granuloma, which have been proposed to be anoxic, and nutritionally starved (Reviewed in [47]). The granulomatous lesion; an in vivo environment encountered by the dormant bacilli, presents a nutritionally deprived and an anoxic environment [125] (Reviewed in [47]). It has been shown that adaptation of $M$. tuberculosis to the in vivo environment involves differential expression of genes that respond to iron limitation, alternative carbon metabolism, and cellular hypoxia [134]. Based on these observations, anaerobic and starved cultures are used as models to study the molecular basis of dormancy. Another study has shown that upon starvation, mycobacterial species such as M. tuberculosis, $M$. kansasii and $M$. pheli, become "chromophobic", whereby they become resistant to staining and can survive for at least two years under starvation [94]. These starved mycobacterial cultures can regain their acid-fast bacilli stain morphology once they are transformed into nutrient rich media [94]. These observations suggest physiological similarities between the $M$. tuberculosis in TB and the studied mycobacterial species in nutritionally starved cultures [94].

An increasing problem in TB treatment is the emergence of multi-drug resistant TB (MDR-TB), and extensively-drug resistant TB (XDR-TB). MDR-TB is resistant to the first line of anti-TB drugs, isoniazid and rifampicin [147]. XDR-TB is resistant to MDR-TB drugs (isoniazid and rifampicin), one of the injectable aminoglycosides i.e. amikacin, kanamycin, or capreomycin, and one of the fluoroquinolones [147]. The WHO has estimated that there are 490,000 new cases of MDR-TB and 40,000 new cases of XDR-TB each year [147]. There is an urgent need to discover new more effective anti-TB drugs to reduce the treatment time, treat cases of MDR-TB and XDR-TB, and to treat latent TB infections. 


\section{Chapter 1: Introduction}

\subsection{Finding new drug targets to treat TB}

To develop new drugs for the treatment of $\mathrm{TB}$, new targets are required. The bacterial signal transduction system is a possible target against which new drugs can be developed for the treatment of TB. The signal transduction system senses changes in the bacterium's external environment and regulates a range of important processes including nutrient uptake, environmental stress tolerance, antibiotic resistance, and virulence gene expression [9]. The most widely known nucleotides involved in signal transduction systems are cyclic nucleotides.

Cyclic nucleotides are components of a wide variety of different signalling pathways [9]. 3', 5'- adenosine cyclic monophosphate (cAMP) and 3', 5'-guanosine cyclic monophosphate (cGMP) were the first cyclic nucleotides discovered, about 50 years ago (Reviewed in [62]). Both are known as "key universal second messengers" [9], and function in signal transduction pathways controlling many important cellular processes such as chemotaxis, differentiation, and proliferation (Reviewed in [62]). In some bacterial pathogens, they are also necessary for sensing and responding to the host environment [9]. Studies on cAMP expression in mycobacteria grown in the presence of glycerol have revealed an intracellular expression level 100-200 fold higher than in Escherichia coli [96]. The expression levels of cAMP in M. smegmatis

are a few hundred-fold higher than in Saccharomyces carlbergensis and Chlamydomonas reinhardtii (Reviewed in [89]). cAMP was also found to be present in the growth media of mycobacteria culture, suggesting an ability to secrete cAMP (Reviewed in [89]). 
Studies on $M$. tuberculosis infected macrophages have shown that cAMP levels increase in macrophages upon infection [84]. Other studies have demonstrated cAMP to be involved in reducing phagocyte activity in Bordetella pertusis and Bacillus anthracis $[28,146]$. Similar results have been proposed in studies on cAMP in Mycobacterium microti and Mycobacterium bovis BCG [71, 84]. However, there is no clear evidence to show that the observed cAMP in infected macrophages is mycobacterial in origin and/or is produced by the host.

Studies based on the role of cyclic nucleotides in bacterial adaptive responses to nutritional stress have demonstrated a mechanism called the 'stringent response' [95]. A feature of the stringent response is the accumulation of the nucleotide guanine tetraphosphate, ppGpp. This nucleotide down regulates the expression of RNA stabilizing proteins, ribosomal proteins and lipid biosynthetic enzymes, while it activates other proteins, which are involved in stress resistance [95]. An accumulation of ppGpp has been shown to occur in M. tuberculosis, M. leprae and M. smegmatis [95], suggesting a ppGpp-like nucleotide may act as a key regulator of $M$. tuberculosis survival during the stationary phase [95].

Even though studies on cyclic nucleotides such as cAMP have been carried out in the mycobacterium sp. M. smegmatis, M. tuberculosis, M. phlei, and M. bovis BCG [85, 96, and 97], the mechanisms behind cyclic nucleotide signalling in mycobacteria are not fully understood. However, using anti-TB drugs targeting cAMP and/or cGMP may not be suitable because of their wide functional importance in host eukaryotic cells. 
Recently, a new bacterial cyclic nucleotide, cyclic di-guanosine monophosphate (c-di-GMP), has been described. This nucleotide act as a "second messenger" during biofilm formation, virulence, motility, and survival signalling pathways $[15,24,25,26,36,37,42,44,49,55,57,72,74,75$, and 82$]$. Its presence in eukaryote cells has not been recorded to date. Furthermore, no studies have been carried out to address the role of c-di-GMP in $M$. tuberculosis pathogenesis and survivability under environmental stress response.

Investigating the role of c-di-GMP in $M$. tuberculosis could provide important knowledge of the mycobacterial survival signalling pathways, drug resistance and stress response. It may also offer new hopes for novel drugs targeting TB epidemic infection, and lead to the creation of new anti-TB drugs that target mechanisms such as the survival-signalling pathways and the stress response.

\subsection{The role of cyclic di-GMP in bacteria}

In the 1980s, a third cytoplasmic cyclic nucleotide, c-di-GMP (Figure 1.3.1), was discovered by Moshe Benziman [11] (Reviewed in [111]). His work was based on the process involved in controlling glucose condensation into cellulose polymers in Acetobacter xylinum (Reviewed in [111]). It was demonstrated that c-di-GMP was controlled through the opposing activities of the two enzymes di-guanylate cyclase (DGC) and phosphodiesterases A (PDEA) [129]. 
New studies on c-di-GMP provide evidence that the novel c-di-GMP is a "common ubiquitous signalling system" that is used in many bacteria (Reviewed in [109] and [110]).

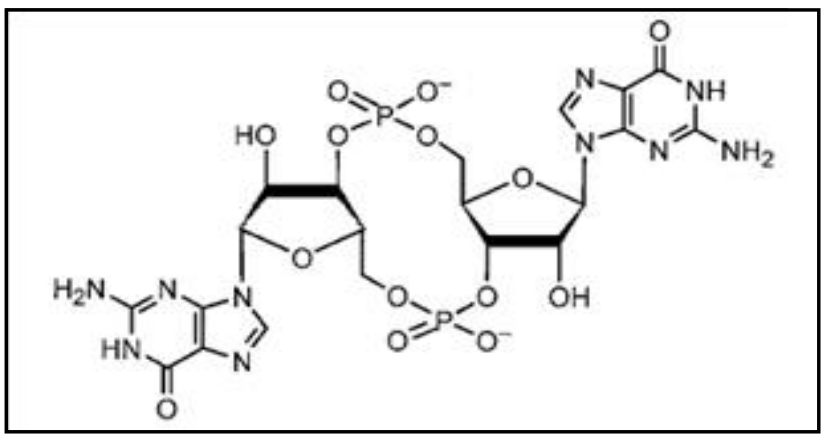

Figure 1.3.1 - Structure of c-di-GMP Chemical structure of c-di-GMP second messenger. Figure was reproduced with permission from the author [112].

All of the studies investigating the function of c-di-GMP in bacteria conclude that high cellular levels of c-di-GMP causes attachment, aggregative behaviour, biofilm formation, and the production of exopolysaccharide [68 and 130] (Reviewed in [62], [109] and [111]). Decreased cellular levels of c-di-GMP promote motility and the production of virulence factors in humans, animals, and plant pathogens $[2$, $15,16,17,24,36,37,42$, and 44].

Biofilm formation in mycobacteria spp. such as $M$. fortuitum, $M$. marinum and M. smegmatis has been recorded previously [48, 49, and 50] (Reviewed in [100]). It has been proposed that the formation of biofilm in mycobacteria could contribute to its immune system resistance, as well as to antibiotic resistance [22 and 117]. 
Biofilm formation in other bacteria such as Bordetella pertussis and Vibrio cholerae, is believed to cause chronic infection in the host [42 and 48] (Reviewed in [100]). Virulence genes encoding c-di-GMP have been discovered in Salmonella typhimurium, V. cholerae, B. pertussis and Pseudomonas aeruginosa $[5,55,76,77$, 135, and 137].

It is possible that M. tuberculosis uses c-di-GMP as a signalling molecule to sense its surrounding environment, and to escape phagosytosis by aiding in the process of blocking the maturation of mycobacterial phagosome into the phagolysosome either directly or indirectly. It is also possible that c-di-GMP is involved in the mechanism of the latent state characteristic of $M$. tuberculosis in that once the host becomes immuno-compromised c-di-GMP sends down-stream signals to activate many transcriptional pathways that lead to active TB in the host. These possibilities lead to the hypothesis that c-di-GMP acts as a global regulator in mycobacteria.

\subsection{The synthesis and degradation of c-di-GMP}

Proteins involved in the synthesis and degradation of c-di-GMP contain GGDEF and/or EAL domains. In some cases, GAF and/or PAS domains are found next to GGDEF and/or EAL domains [113] (Reviewed in [109], [110] and [112]). The domain names originate from the conserved sequence motifs Gly-Gly-Asp-GluPhe (GGDEF) (Reviewed in [114]), and Glu-Ala-Leu (EAL) [118]. 


\section{Chapter 1: Introduction}

The GAF domain name originates from c-GMP-specific and stimulated phosphodiesterase/Adenylate cyclase (Anabaena)/ FhlA (E. coli) [116]. PAS and GAF domains are structurally similar, but are unrelated in amino acid sequences leading to different ligand-binding molecules [116].

The GGDEF domain was discovered in Caulobacter crescentus [53]. This bacteria has a protein named PleD that contains a C-terminal GGDEF domain and two N-terminal receiver domains (Reviewed in [62]). The PleD protein function as a response regulator, controlling cell differentiation in the swarmer (flagellated form) to stalked (surface attached form) cell transition (Reviewed in [41]). Biochemical examinations conducted on the PleD protein of C. crescentus gave direct biochemical evidence that the GGDEF domain possesses DGC activity (the GGDEF domain convert two molecule of GTP to c-di-GMP) [101] (Figure 1.3.2). Other in vitro biochemical analyses in bacteria such as S. typhimurium, Pseudomonas fluorescens, Yersinia pestis, V. cholerae, and E. coli, strengthened the association of the GGDEF domain proteins with DGC activity [68, 72, 75, 82, and 122]. Other studies have demonstrated that over-expression of genes encoding GGDEF domain proteins increased cellular levels of c-di-GMP [101, 122, and 136].

The EAL domain was discovered in a protein named BvgR, a repressor of virulence gene expression, in B. pertussis [87]. The regulation of virulence factors in B. pertussis depends on the bvg locus, which encodes BvgA and BvgS [87]. It has been proposed that the BvgS protein response to environmental signals and interacts with BvgA (a transcriptional regulator) [87]. 


\section{Chapter 1: Introduction}

When genes encoding the "major virulence factors" are activated, virulence-response genes are turned off in a BvgR-dependent manner [87]. This observation has concluded BvgR to be part of signal transduction system in B. pertussis [87]. Later studies of A. xylinum found similar domains to that of BvgR named EAL domains, due to their conserved residues. This EAL domain in A. xylinum was found to be located next to a GGDEF domain that regulates cellulose synthesis [136].

Studies conducted on genes encoding only EAL domains demonstrated a decreased level of cellular c-di-GMP; and gave indirect evidence for EAL domain proteins as having a PDE-A activity [122, 131, 136, and 137]. Biochemical studies based on proteins containing EAL domains gave direct evidence of EAL domains as having a PDE-A activity [24, 25, and 118] (Rviewed in [109]). Therefore proteins with GGDEF domains have a DGC activity (synthesises c-di-GMP), and proteins with EAL domains have a PDE-A activity (degrades c-di-GMP) (Figure 1.3.2).

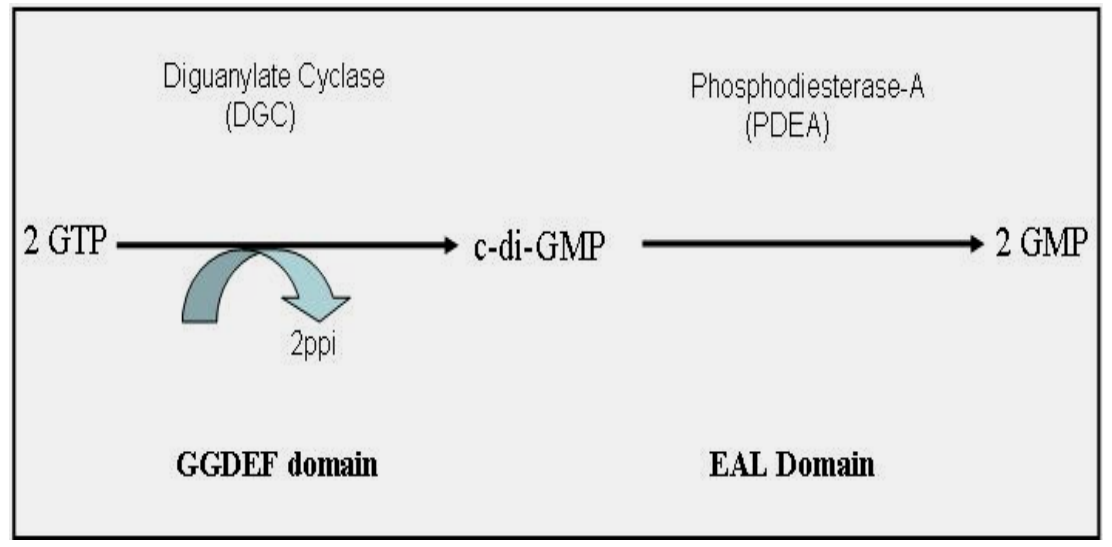

Figure 1.3.2 - Synthesis and degradation of c-di-GMP: GGDEF domains have DGC activity that produces c-di-GMP from 2-GTP. EAL domains contain PDE-A activity that hydrolyses c-di-GMP to the linear form pGpG (2 GMP). 


\section{Chapter 1: Introduction}

The GAF domain was originally described as a "non-catalytic cGMP-binding domain" conserved in cyclic nucleotide phosphodiesterases [20]. Later studies described it as "cyanobacteria adenylate cyclase" and histidine kinase [6], and it is often found linked with GGDEF and/or EAL domains [6]. Proteins with GAF domain are shown to have functions in gene regulation [6], light detection and signalling in plants and cyanobacterial phytochromes [121] (Reviewed in [89]), nitrogen fixation in bacteria [65], feedback control of a cyanobacterial adenylyl cyclase by cAMP-binding [69], and the two-component sensor histidine kinase in viruses, bacteria, and plants (Reviewed in [41] and [139]). Therefore most of the literature based on c-di-GMP and cell signalling has shown GAF domains to play role in signal inputs from the surrounding environments [19, 36, 57, 66, 70, 103, and 145], and/or function in adjusting GGDEF-EAL domain activities (Reviewed in [132]).

No previous studies have been carried out on c-di-GMP and its domains (GAF-GGDEF-EAL) in mycobacteria. c-di-GMP seems to be ubiquitous in the kingdom of prokaryotes with some bacteria having multiple copies of GGDEF-EAL domains. For example, $P$. aeruginosa has 17 GGDEF, 5 EAL and 16 GGDEF-EAL domain proteins (Reviewed in [41]), and V. cholerae has 31 GGDEF, 22 EAL, and 10 GGDEF-EAL domain proteins (Reviewed in [41]). The M. tuberculosis genome contains only one protein with GAF-GGDEF-EAL domains designated as Rv1354c [7], and three proteins containing GAF domain i.e. Rv1354c, Rv2027c, and Rv3132c [7]. Studies have been conducted on the GAF domain of Rv3132c protein, and it was found to be activated through the binding of molecules such as oxygen and nitric oxide [116]. 
Since c-di-GMP is involved with proteins containing GGDEF and/or EAL domains, Rv1354c was investigated in this study using M. bovis and M. smegmatis as the model organism. Rv1354c was listed as a "conserved hypothetical protein", it consists of 623 amino acids, and its function in mycobacteria is not known. The focus of this study was to investigate possible role of Rv1354c in M. smegmatis responses to different environmental stresse conditions. Sense and antisense experiments were conducted in M. smegmatis. Using nutrient-rich, or carbon-, oxygen-, or phosphorous-limited conditions, the altered expression of Rv1354c, in M. smegmatis, was examined in term of cell growth effect. Proteomic analysis was carried out to determine possible differential protein expression under limited conditions. Mutagenesis experiments, such as overlap PCR were applied to construct the knock-out of Rv1354c strain in M. bovis and M. smegmatis.

Most of the work involved with M. bovis was carried out at the AgResearch PC3 laboratory (Wallaceville, New Zealand). Since M. smegmatis is less virulent all of the work was carried out at PC2 Microbiology laboratory (Victoria University of Wellington, New Zealand). 


\subsection{Aims}

\subsubsection{Determine whether a c-di-GMP enzyme is encoded by $M$. tuberculosis genome}

To find out whether the M. tuberculosis genome encodes c-di-GMP enzyme, the M. tuberculosis genome will be searched for GGDEF and or/EAL domains using TIGERFAMs database.

\subsubsection{Construction of sense and antisense c-di-GMP strains in M. smegmatis}

To determine the possible role of c-di-GMP in $M$. smegmatis a sense and an antisense strain will be constructed. The construction of c-di-GMP sense and antisense strains will be conducted by inserting the c-di-GMP PCR fragment into the expression vector pMind. Tetracycline will be used to conditionally control the expression of the sense and antisense fragments in $M$. smegmatis.

1.5.3 Investigate the effect of the changed expression of c-di-GMP sense and antisense on the physiology of $M$. smegmatis

To examine the presence of any growth effect caused by the altered expression of c-di-GMP in M. smegmatis, Hartman de Bont (HdeB) oxygen, nitrogen, phosphorous, and carbon minimal growth media will be used. 


\subsubsection{Proteomic analysis of MSMEG_2196 in HdeB growth limited media}

To determine possible differential protein expression between the c-di-GMP sense and antisense strains under limited growth conditions, proteomic analysis will be carried out on any significant growth effect observed in the HdeB limited growth assays.

\subsubsection{Mutation of c-di-GMP, in $M$. bovis and $M$. smegmatis, respectively}

To construct a knock-out of c-di-GMP in $M$. bovis and M. smegmatis, overlap PCR extension will be used. The c-di-GMP deletion fragments will be inserted into the suicide pUHA778 plasmid to generate the knockout vector.

To test c-di-GMP for virulence and pathogencity in $M$. bovis, mice will be used where they will be infected with the $M$. bovis c-di-GMP mutant. The $M$. smegmatis c-di-GMP mutant will be examined for pathogencity in macrophages, and for growth and developmental effects in a series of growth curves under limited growth conditions in the log and stationary phase. 


\section{Materials and Methods}

\subsection{Bacterial strains, plasmids and culture conditions}

The bacterial strains used in this study were $M$. smegmatis $\mathrm{mc}^{2} 155$ (Victoria University), and $M$. bovis WAg203, provided by AgResearch (Wallaceville, New Zealand). M. smegmatis $m c^{2} 155$ cultures were grown overnight in Luria-Bertani broth [LB] (Invitrogen, Auckland, New Zealand) at $37{ }^{\circ} \mathrm{C}$ (composition (g/L) - 10.0 peptone from casein, 5.0 yeast extract, $10.0 \mathrm{NaCl}$ ) or on LB agar [LA] (Invitrogen, Auckland, New Zealand) (compostion [g/L] - 10.0 peptone from casein, 5.0 yeast extract, 10.0 $\mathrm{NaCl}_{2}, 12.0$ Agar). When grown in LB, 0.1\% Tween 80 (MERCK, New Zealand) was used to avoid clumping of the M. smegmatis cells. E. coli DH5 $\alpha$ cells were used and they were grown at $37^{\circ} \mathrm{C}$ in LB and/or LA.

The Cloning vectors used were pGEM-T Easy (Promega, Auckland, New Zealand) and pCR2.1 (Invitrogen, Auckland, New Zealand), following manufacturer's instructions. All work with these vectors was carried out on LA with Ampicillin (Sigma-Aldrich, New Zealand) at $200 \mu \mathrm{g} / \mathrm{mL}$, IPTG (BioLine, Australia) at $100 \mu \mathrm{g} / \mathrm{mL}$ and Xgal (BioLine, Australia) at $40 \mu \mathrm{g} / \mathrm{mL}$. pMind (Appendix 6.1.2) vector was used to construct the antisense and sense strains as described previously [14]. This plasmid was grown using LA/LB with Kanamycin (Sigma-Aldrich, New Zealand) at $50 \mu \mathrm{g} / \mathrm{mL}$ and Hygromycin (Sigma-Aldrich, New Zealand) at $50 \mu \mathrm{g} / \mathrm{mL}$, and incubated at $37^{\circ} \mathrm{C}$. pUHA778 with Pac I site (Appendix 6.1.1) was used as the knock out vector, obtained from AgResearch (Wallaceville, New Zealand). Work on this plasmid was carried out mainly on LA and /or on LB with Ampicillin at $100 \mu \mathrm{g} / \mathrm{mL}$ and Hygromycin at $50 \mu \mathrm{g} / \mathrm{mL}$, grown at $37^{\circ} \mathrm{C}$. 
Chapter 2: Materials and Methods

Hartman-de Bonts (HdeB) minimal media [125] was used to perform growth assays under limited conditions.

\subsection{Bioinformatics of c-di-GMP in the genome of mycobacteria}

A literature search was carried out to find genes involved with c-di-GMP in bacteria such as $V$. cholerae and A. xylinum. Most of the proteins involved in the synthesis and degradation of c-di-GMP contained GAF, GGDEF, and or EAL domains.

The M. tuberculosis genome was searched for a GGDEF domain using "The Institute for Genomic Research" (TIGR) website [133] with text search. Rv1354c was the only gene in $M$. tuberculosis genome containing the three domains GAF-GGDEFEAL. To find homologue gene of Rv1354c in M. smegmatis and M. bovis, Rv1354c gene sequences were blasted against the $M$. smegmatis and $M$. bovis genome, respectively, using (www.vge.ac.uk/blast/blast.cgi) database. The orthologue of Rv1354c in M. bovis is Mb1389c, and in M. smegmatis is MSMEG_2196. Rv1354c and Mb1389c are annotated as "conserved hypothetical protein" in $M$. tuberculosis and $M$. bovis, respectively. MSMEG_2196 is annotated as a "sensory box/response regulator" protein. Pfam (www.sanger.ac.uk/Software/Pfam/) was also used to run the selected M. bovis and M. smegmatis proteins for domain structure view. 
Chapter 2: Materials and Methods 
Chapter 2: Materials and Methods

\subsection{Altered expression of MSMEG_2196 in M. smegmatis}

\subsubsection{Preparation of genomic DNA}

Throughout this study, the cultured cells, either sub-cultured from LB or inoculated from LA plates, were grown into $3 \mathrm{~mL}$ of $\mathrm{LB}$ and incubated at $37{ }^{\circ} \mathrm{C}$ overnight. The cultures were then collected by centrifuging at 7,000 x g for $15 \mathrm{mins}$, and genomic DNA was extracted using the phenol-chloroform protocol as follow: cells were resuspended in $400 \mu \mathrm{L}$ of Tris-EDTA buffer (TE buffer). The resuspension was followed by the addition of lysozyme (Sigma-Aldrich, New Zealand) to a final concentration of $2 \mathrm{mg} / \mathrm{mL}$; the cells were then mixed gently and incubated at $37^{\circ} \mathrm{C}$ in a water bath for 30 mins. SDS (Invitrogen, New Zealand) was added at a final concentration of $1 \%$ followed by the addition of $0.2 \mathrm{mg} / \mathrm{mL}$ of proteinase K (Sigma-Aldrich, New Zealand). Then the cell suspension was mixed and incubated in a water bath set at $65^{\circ} \mathrm{C}$ for 15 mins. $400 \mu \mathrm{L}$ of Phenol (MERCK, Australia) was added to the cell suspension and mixed. Then the suspension mixture was centrifuged at $16,000 \mathrm{x}$ g for $10 \mathrm{mins}$, after which the upper aqueous layer was removed to a fresh microfuge tube leaving behind the white precipitate. $400 \mu \mathrm{L}$ of chloroform (Ajax Finechem Ltd., New Zealand) was added to the aqueous layer and mixed, then centrifuged at $16,100 \mathrm{x}$ g for another $10 \mathrm{mins}$. The aqueous layer was once again removed to a fresh microfuge tube. $99 \%$ cold ethanol (Ajax Finechem Ltd., New Zealand) was added at 2.5 volume, and sodium acetate, pH 5.2 (SigmaAldrich, New Zealand) was added at a final concentration of $0.1 \mathrm{M}$. The solution was then mixed and centrifuged at $16,100 \times$ g for 20 mins. $400 \mu \mathrm{L}$ of cold $70 \%$ ethanol was then added to the supernatant and centrifuged at $16,100 \mathrm{x} g$ for 5 mins. The pellets were then allowed to dry in a heating block set at $37^{\circ} \mathrm{C}$ until dry. $200 \mu \mathrm{L}$ of 
Chapter 2: Materials and Methods

sterile-distilled water was added and the DNA stored at $4{ }^{\circ} \mathrm{C}$. This protocol can also be found in Sambrook and Russell (Volume 1- 6.4-6.11) [115].

\subsubsection{Preparation of Mycobacterium smegmatis $\mathrm{mc}^{2} 155$ electroporation- competent cells}

M. smegmatis $\mathrm{mc}^{2} 155$ electroporation-competent cells were prepared as outlined in Sambrook and Russell (Volume 1, 1.12-1.14) [115]. Briefly, M. smegmatis cells were inoculated from LA plates into $10 \mathrm{~mL} \mathrm{LB}$ (no antibiotics) and incubated overnight at $37^{\circ} \mathrm{C}$. The next day, $1 / 5$ of the overnight culture were subinoculated into $100 \mathrm{~mL} \mathrm{LB}$ (no antibiotics) and incubated during the day at $37{ }^{\circ} \mathrm{C}$, cells were harvested when the $\mathrm{OD}_{600}$ absorbance was between 0.5 and 1.0. Cells were divided into two $50 \mathrm{~mL}$ Falcon tubes (Labserve, New Zealand), then incubated on ice for $20 \mathrm{mins}$, and then pelleted for $15 \mathrm{mins}$ at $9,000 \mathrm{xg}$ at $4{ }^{\circ} \mathrm{C}$. The pellets were resuspended in $50 \mathrm{~mL}$ of $10 \%$ cold glycerol and pelleted for 15 mins at 9,000 x g at $4{ }^{\circ} \mathrm{C}$, once again, the cells were resuspended in $20 \mathrm{~mL}$ of $10 \%$ cold glycerol, and cells were pelleted for another 15 mins at $9,000 \times \mathrm{g}$ at $4{ }^{\circ} \mathrm{C}$. Finally, the pellets were resuspended in $2 \mathrm{~mL}$ of $10 \%$ glycerol and $100 \mu \mathrm{L}$ aliquots were dispensed into chilled microfuge tubes and stored at $-80{ }^{\circ} \mathrm{C}$ or used immediately for electroporation. 
Chapter 2: Materials and Methods

\subsubsection{PCR of MSMEG_2196}

The final concentration of PCR reagents used, to amplify MSMEG_2196 gene, were $0.16 \mathrm{mM}$ dNTPs, $0.8 \mu \mathrm{M}$ of each primer (Ms_2196 F1 and Ms_2196 R1), 2.5 $\mathrm{mM} \mathrm{MgSO}_{4}, 1 \times$ PCR reaction buffer, $0.05 \mathrm{U} / \mu \mathrm{L}$ Platinum Taq polymerase. DMSO, when used, was added at a concentration of $2.5 \%$, respectively (all of the PCR reagents and primers were purchased from Invitrogen, Auckland, New Zealand). $3.0 \mu \mathrm{L}$ of template DNA $(0.3 \mu \mathrm{g})$ was added to a $25 \mu \mathrm{L}$ reaction. The PCR conditions are shown in table 2.3.1 and primers in table 2.3.2.

All of the PCR products were run on $1 \%$ low melting temperature agarose gels (Roche Applied Science, Auckland, New Zealand) with ethidium bromide (1 $\mu \mathrm{g} / \mathrm{mL}$ ) for DNA detection. For PCR product purification, the Roche DNA gel purification kit (Roche Applied Science, Auckland, New Zealand) was used following the manufacturer's instructions.

\begin{tabular}{|l|l|l|}
\hline Steps & $\begin{array}{l}\text { Temperature } \\
\left({ }^{\circ} \mathbf{C}\right)\end{array}$ & $\begin{array}{l}\text { Time } \\
(\mathbf{m i n s})\end{array}$ \\
\hline 1. Denaturation & 94 & 5.0 \\
\hline 2. Annealing & 50 & 1.0 \\
\hline 3. Extension & 68 & 2.0 \\
\hline 4. Steps 1-3 repeat 30 times & & \\
\hline 5. Extension & 68 & 10 \\
\hline
\end{tabular}

Table 2.3.1: PCR conditions used to amplify MSMEG_2196 fragment 
$\underline{\text { Chapter 2: Materials and Methods }}$

\begin{tabular}{|l|l|l|}
\hline $\begin{array}{l}\text { Primer } \\
\text { name }\end{array}$ & $\begin{array}{l}\text { Enzyme } \\
\text { site }\end{array}$ & Sequence \\
\hline Ms_2196 F1 & Pac I & CTAGTTAATTAAGACGTCGTAACAGGGCTGAGC \\
\hline Ms_2196 R1 & Pac I & CTAAGTTAATTAAGGGGCGCCGCACTCACATGCG \\
\hline
\end{tabular}

Table 2.3.2: MSMEG_2196 antisense and sense construction primers. Nucleotides in bold letters represent the enzyme site.

\subsubsection{Ligation of MSMEG_2196 into pCR 2.1 cloning vector}

$3 \mu \mathrm{L}$ of the gel purified MSMEG_2196 PCR product was ligated into pCR 2.1 cloning vector (Invitrogen, Auckland, New Zealand), ligation reactions were performed with $0.4 \mathrm{U} / \mu \mathrm{L}$ of T4 DNA ligase (Invitrogen, New Zealand), 1x ligation buffer (Invitrogen, Auckland, New Zealand), and $1 \mathrm{ng} / \mu \mathrm{L}$ of pCR2.1 (Invitrogen, New Zealand) in a total volume of $10 \mu \mathrm{L}$. The ligation reaction was incubated at 14 ${ }^{\circ} \mathrm{C}$ for 4 hrs.

\subsubsection{Heat shock transformation of MSMEG_2196-pCR2.1 fragment into E. coli DH5a cells}

To transform the MSMEG_2196-pCR2.1 fragment, E. coli DH5a calciumcompetent cells were used. Standard heat shock transformation protocol was used as follow; $50 \mu \mathrm{L}$ of $E$. coli DH5 $\alpha$ cells were first thawed on ice for 5 mins, $280 \mu \mathrm{L}$ of cold $0.1 \mathrm{M} \mathrm{CaCl}_{2}$ (Ajax Finechem Ltd., New Zealand) was added, this was then added to the ligation mixture and incubated on ice for 20 mins. 
Chapter 2: Materials and Methods

The cells were heat-shock treated at $42{ }^{\circ} \mathrm{C}$ for 90 secs, then placed on ice for five mins, $280 \mu \mathrm{L}$ of LB was added to the cells and incubated at $37{ }^{\circ} \mathrm{C}$ for $1 \mathrm{hr}$. The transformation mixture was centrifuged at $16,100 \mathrm{x}$ g for $50 \mathrm{secs}$, excess supernatant was discarded leaving behind $100 \mu \mathrm{L}$ for plating, and the cells were re-suspended into this. The cells were then plated out onto LA Amp 200, IPTG $_{100}$, X-gal 40 plates and left at $37^{\circ} \mathrm{C}$ for an overnight incubation.

\subsubsection{Midipreparation, purification, and stock culture of MSMEG_2196-pCR2.1 plasmid DNA}

White colonies were picked from the overnight LA Amp 200, IPTG $_{100}$, X-gal 40 plates (from 2.3.5), and grown in $100 \mathrm{~mL}$ of $\mathrm{LB}$ containing $\mathrm{Amp}_{200}$ at $37{ }^{\circ} \mathrm{C}$ overnight. Midipreprations of plasmid DNA were carried out using the Invitrogen S.N.A.P ${ }^{\text {TM }}$ midiprep kit (Invitroge, Auckland, New Zealand) following manufacturer's instructions. The Invitrogen PureLink ${ }^{\mathrm{TM}}$ PCR (Invitrogen, Auckland, New Zealand) purification kit was used to purify the plasmid following manufacturer's instructions. Stock cultures were prepared by adding $400 \mu \mathrm{L}$ of $50 \%$ glycerol to $600 \mu \mathrm{L}$ of the cell culture and stored at $-80^{\circ} \mathrm{C}$. 
Chapter 2: Materials and Methods

\subsubsection{Plasmid digestion and gel purification of MSMEG_2196-pCR2.1}

The MSMEG_2196-pCR2.1 midipreparations were digested with Pac I NEB (BioLab, New Zealand) enzyme to check for insertion. The digestion reaction contained $1 \mathrm{U} / \mu \mathrm{L}$ of Pac I (concentration at $10 \mathrm{U} / \mu \mathrm{L}$ ), 1 X NEB Buffer 1 , and $20 \mu \mathrm{L}$ of plasmid in $25 \mu \mathrm{L}$ total reaction. The digestion reactions were left at $37^{\circ} \mathrm{C}$ for overnight incubation, and were run on a $1 \%$ agarose gel.

For plasmid purification, the digested reaction was extracted from $1 \%$ agarose gel and purified using Roche DNA purification kit (Roche, Auckland, New Zealand) following manufacturer's instructions. The purified digested fragment was ready to be ligated into pMind expression vector.

\subsubsection{Sequencing of MSMEG_2196-pCR2.1 fragment}

The digested plasmids (2.3.7) with the right size insert, were first quantified using either the Quanti-iT ${ }^{\mathrm{TM}}$ PicoGreen ${ }^{\circledR}$ (Invitrogen, Auckland, New Zealand) DNA quantification method, and/or the NanoDrop ND-1000 spectrophotometer (Global Science and Technology Ltd., Auckland, New Zealand) method following the manufacturer's instructions. For sample sequencing, the DNA concentrations of the midipreparations were adjusted to $300 \mathrm{ng} / \mathrm{mL}$ in a total volume of $14 \mu \mathrm{L}$, and sent to the Allan Wilson Centre in Palmerstorn North, New Zealand for sequencing.

The chromatograms of the sequences were analyzed using Vector NTI software (Invitrogen, Auckland, New Zealand). 
Chapter 2: Materials and Methods

Corrections of the sequences were carried out in term of best fit such as length of sequence and identity, minimum number of mutations and clarity of the chromatogram, the best ones were then selected for further work.

\subsubsection{Ligation of MSMEG_2196 into the pMind expression plasmid}

Pac I digested and column purified pMind plasmid was provided by Shahista Nisa, Victoria University of Wellington, New Zealand. The ligation reaction consisted of $1 \mu \mathrm{L}$ of Pac I digested pMind, 1x ligation buffer 1 (BioLabs, Auckland, New Zealand), $3 \mu \mathrm{L}$ of Pac I digested and gel purified MSMEG_2196-pCR2.1 fragment, and $0.4 \mathrm{U} / \mu \mathrm{L}$ T4 DNA ligase in a total ligation volume of $10 \mu \mathrm{L}$. The ligation reaction was incubated at $14^{\circ} \mathrm{C}$ overnight.

MSMEG_2196-pMind ligation was transformed into E. coli DH5 $\alpha$ cells as in 2.3.5. The only notable difference was that the cells were plated onto LA-Hyg ${ }_{50}$ $\mathrm{Kan}_{50}$ plates and incubated at $37^{\circ} \mathrm{C}$ for 2 days.

\subsubsection{Insert and orientation check of MSMEG_2196 into pMind plasmid}

Single white colonies, from 2.3.9, were inoculated into $3 \mathrm{~mL}$ of LB broth containing $\mathrm{Kan}_{50} \mathrm{Hyg}_{50}$ and incubated at $37{ }^{\circ} \mathrm{C}$ overnight for midipreprations as in 2.3.6. To check for the insertion of MSMEG_2196 fragment into pMind, midipreparations were digested with $0.25 \mathrm{U} / \mu \mathrm{L}$ of Pac I, 1x NEB buffer 1 (BioLabs, Auckland, New Zealand), and $10 \mu \mathrm{L}$ of plasmid in a $20 \mu \mathrm{L}$ total reaction, the 
Chapter 2: Materials and Methods

digestion reaction was then run on a $1 \%$ agarose gel. Culture cells with insert in them were prepared and stored at $-80^{\circ} \mathrm{C}$ as in 2.3.6.

To check for the orientation of MSMEG_2196 in pMind, a PCR was conducted using the TetRO primers, sequences in table 2.3.3. Similar PCR conditions to that in 2.3.3 were followed except that $1 \mu \mathrm{L}$ of each of the TetRO-F, Ms_2196/F1, and TetRO-R, Ms_2196/R1 primers were used, for the PCR regimen see table 2.3.1. The PCR reaction was run on $1 \%$ agarose gel and sent to the Allan Wilson Centre for sequencing as in 2.3.8. From the chromatograms of the sequences, sense and antisense orientations were confirmed.

\begin{tabular}{|l|l|}
\hline Primer name & Sequence \\
\hline TetRO-F & GCTCTAGATCACGATTCGCTCGAGGTC \\
\hline TetRO-R & CGGGATCCTGTCAGGATTCCACGATGAG \\
\hline
\end{tabular}

Table 2.3.3: pMind TetRO primers used to determine the sense and antisense orientations of MSMEG_2196 fragment in pMind. 
Chapter 2: Materials and Methods

2.3.11 Electroporation of MSMEG_2196-antisense/sense-pMind into $M$. smegmatis

The MSMEG_2196-pMind midipreparations were prepared for electroporation as follow: MSMEG_2196-pMind midiprep was concentrated by spinning $200 \mu \mathrm{L}$ in vacuum down to $30 \mu \mathrm{L}$. The concentrated DNA was dialyzed on a nitrocellulose membrane, floating on $10 \mathrm{~mL}$ sterile distilled water for 30 mins then quantified using NanoDrop ND-1000 Spectrophotometer; DNA final concentration was $0.95 \mu \mathrm{g} / \mu \mathrm{L}$.

1, 5, and $10 \mu \mathrm{L}$ of the dialyzed MSMEG_2196-pMind was electro-porated into $100 \mu \mathrm{L}$ M. smegmatis $\mathrm{mc}^{2} 155$ competent cells made up to $400 \mu \mathrm{L}$ with $20 \%$ glycerol. The electroporation was carried out at $2.5 \mathrm{kV}$ using Bio-Rad E. coli pulser electroporator (Bio-Rad, Auckland, New Zealand). $400 \mu \mathrm{L}$ of LB was added to the electroporation mix, and the cells were left to recover at $37^{\circ} \mathrm{C}$ overnight. The cells were then plated onto LA-Hyg ${ }_{50} \mathrm{Kan}_{50}$ plates and incubated at $37^{\circ} \mathrm{C}$ for 1 week. For control purposes, $10 \mu \mathrm{L}$ of empty pMind plasmid was also transformed into $M$. smegmatis cells.

M. smegmatis transformatn colonies were inoculated into $3 \mathrm{~mL}$ of LB-Hyg ${ }_{50}$ $\mathrm{Kan}_{50}$ with $0.1 \%$ Tween 80 and incubated at $37{ }^{\circ} \mathrm{C}$ for two days, after which $-80{ }^{\circ} \mathrm{C}$ freezer stock were made as in 2.3.6. 


\subsection{Investigating growth effects of MSMEG_2196-antisense/sense}

\subsubsection{Growth curves}

Growth curves were carried out in rich media using LB broth, and in nutrientlimited media using Hartman de Bonts (HdeB) carbon $\left(\mathrm{C}^{-}\right)$, oxygen $\left(\mathrm{O}^{-}\right)$, phosphorous $\left(\mathrm{P}^{-}\right)$, and nitrogen $\left(\mathrm{N}^{-}\right)$limited conditions,(appendix 5.2). The cultures in $\mathrm{HdeB} \mathrm{O}^{-}$limitation were grown in sealed (Suba seals) $250 \mathrm{~mL}$ flasks containing $150 \mathrm{~mL}$ of HdeB media giving a headspace ratio of 1.67. For $\mathrm{OD}_{600}$ sample readings, samples were taken by inverting the flask and inserting a fine 25-gauge needle through the Suba seal and extracting the sample by using $1 \mathrm{~mL}$ syringe (Bacton Dickinson, Auckland, New Zealand).

M. smegmatis $\mathrm{mc}^{2} 155$ wild type (M. smeg WT), M. smegmatis/pMind (Ms/pMind), $\quad M . \quad$ smegmatis/pMind-2196-antisense $(\mathrm{Ms} / \mathrm{p} 2196$ AS), and $M$. smegmatis/pMind-2196-sense (Ms/p2196 S) from $-80{ }^{\circ} \mathrm{C}$ stock cultures were streaked out onto the appropriate agar plates and incubated at $37^{\circ} \mathrm{C}$ for 3 days. For starting culture, an inoculating loop of cells from these plates was initially placed into $50 \mathrm{~mL}$ of $\mathrm{HdeB} \mathrm{O}^{-}$media (without the seals) containing appropriate antibiotics, and LB broth (M. smegmatis $\left.\mathrm{mc}^{2} 155\right)$ with no antibiotics and incubated overnight on a shaker at $37^{\circ} \mathrm{C}$ and $200 \mathrm{rpm}$. Next day the $50 \mathrm{~mL}$ culture was sub-cultured into $50 \mathrm{~mL}$ of LB broth, $\mathrm{C}^{-}, \mathrm{O}^{-}, \mathrm{P}^{-}$, and $\mathrm{N}^{-}$HdeB limited media, with appropriate antibiotics to an initial $\mathrm{OD}_{600}$ absorbance of 0.1 .

The growth assays were conducted in triplicates for each strain, and three cultures contained tetracycline $(\mathrm{Tc}+)$ at a final concentration of $40 \mathrm{ng} / \mathrm{mL}$, and three did not (Tc-) for all 4 strains. All cultures were incubated at $37{ }^{\circ} \mathrm{C}$, at $200 \mathrm{rpm}$ and $\mathrm{OD}_{600}$ absorbance readings were taken every $90 \mathrm{mins}$ for $12 \mathrm{hrs}$ and then daily for up 
Chapter 2: Materials and Methods

to two days. Experiments were repeated, where a significant difference (outside of standard error) was observed in any of the HdeB limited media culture.

\subsubsection{Determination of colony forming units}

Colony forming unit (CFU) counting was carried out on $\mathrm{N}^{-}$cell culture where $1 \mathrm{~mL}$ of cells in stationary phase (24 hrs and $48 \mathrm{hrs}$ ) was removed from each culture for further analysis. $100 \mu \mathrm{L}$ of these cells were serial diluted $10^{-1}$ to $10^{-5}$ in $900 \mu \mathrm{L}$ of $0.9 \%$ sterile $\mathrm{NaCl}_{2}$ containing $0.1 \%$ Tween 80 . The $10^{-3}$ to $10^{-5}$ dilutions were plated out onto LA plates (no antibiotics), and incubated for 3 days at $37^{\circ} \mathrm{C}$ and the colonies obtained were counted. 
Chapter 2: Materials and Methods

\subsection{Proteomic analysis of Ms/p2196 antisense grown under nitrogen limited conditions}

\subsubsection{Protein of Ms/p2196 AS and Ms/pMind cells for one-dimension (1D) and two-dimension (2D) gel analysis}

The Ms/p2196 AS and Ms/pMind Cells grown in HdeB $\mathrm{N}^{-}$limited media (Tc+) were harvested at stationary phase (at time 1,800 mins) and cells were pelleted by centrifugation at $8,000 \times \mathrm{g}$ for 20 mins. Each cell pellet (Ms/pMind and Ms/p2196 AS) was re-suspended in $20 \mathrm{~mL}$ of sterile distilled water containing a cocktail of protease inhibitors (Complete, Roche, New Zealand). The solution was mixed, gently, by pipetting up-and-down for approximately 20 mins until the cells were mixed well in the sterile distilled water.

Triton X-100 (Sigma-Aldrich, Auckland, New Zealand) was added to the resuspended pellets at a $2 \%$ final concentration, and incubated with rocking on ice for $1 \mathrm{hr}$. The solution was passed through a French press, Cell disrupture (Thermo Electron Corporation, Global Science and Technology Ltd., New Zealand) six to seven times until the solution was clear. $2 \mathrm{~mL}$ of the resulting lysate (from each cell sample solution) were aliquoted into eppendorf tubes and kept at $-80{ }^{\circ} \mathrm{C}$ (sample A). To the remaining samples, the followings were added: $7 \mathrm{M}$ urea (Gibco BRL, Life Technologies, New Zealand), 2 M thiourea (GE Healthcare, New Zealand); and 70 mM DTT (Sigma-Aldrich, New Zealand). The solution was incubated with rocking for $2 \mathrm{hrs}$ at room temperature. $2 \mathrm{~mL}$ of this solution (sample B) were aliquoted into eppendorf tubes (from each cell sample solution) and kept at $-80{ }^{\circ} \mathrm{C}$. 
Chapter 2: Materials and Methods

The remaining cell lysates were kept at $-80{ }^{\circ} \mathrm{C} . \quad 1 \mathrm{~mL}$ of sample $\mathrm{A}$ and $1 \mathrm{~mL}$ of sample B were concentrated 5-fold using $5 \mathrm{kDa}$ centrome centrifuge tubes (Millipore, Biolab Scientific, New Zealand).

To check for the quality and quantity of protein in the cell lysates, $20 \mu \mathrm{L}$ and 5 $\mu \mathrm{L}$ of 5-fold concentrated sample A and sample B lysates, and $20 \mu \mathrm{L}$ and $5 \mu \mathrm{L}$ of non-concentrated sample A and B lysates were run on SDS-PAGE (Bio-Rad, New Zealand) using a $12 \%$ acrylamide running SDS gel and $4 \%$ acrylamide stacking gel.

2-D Quanti Kit (Amersham, GE Healthcare, New Zealand) was used to quantify protein samples isolated from Ms/p2196 AS and Ms/pMind non-concentrated lysates.

\subsubsection{D and 2D gel electrophoresis analysis of Ms/p2196 antisense expressing strain and Ms/pMind protein samples on $\mathrm{pH}$ 4-7 isoelectric focusing strips}

For 1D gel electrophoresis, $7 \mathrm{~cm}$ Immobiline DryStrips (GE Healthcare, New Zealand) with an immobilized $\mathrm{pH}$ gradient 4-7, were rehydrated overnight in rehydration buffer solution (7 M urea, $2 \mathrm{M}$ thiourea, $2 \%$ Triton X-100, $70 \mathrm{mM}$ DTT, and $0.5 \%$ IPG buffer $\mathrm{pH} 4-7$ ) containing $100 \mu \mathrm{g}$ of Ms/p2196 antisense and Ms/pMind non-concentrated protein sample in a final volume of $150 \mu \mathrm{L}$. Ettan IPGphor Isoelectric focusing unit (Amersham Biosciences, GE Healthcare, New Zealand) was used to perform 1D gel electrophoresis following the manufacturer's instructions, with the exception step 4 in which the running protocol was run at 4,000 V. 
The focused strips were equilibrated, prior to 2D gel electrophoresis, first, in 2 $\mathrm{mL}$ of solution containing 1x LDS sample buffer (GE Healthcare, Auckland, New Zealand) and reducing agent (GE Healthcare, Auckland, New Zealand) in a 2:1 ratio, incubated at room temperature for 15 mins. Then the strips were immersed into a 2 $\mathrm{mL}$ solution containing $1 \mathrm{x}$ LDS sample buffer and $0.05 \mathrm{~g}$ of Iodoacetamide (GE Healthcare, New Zealand), and incubated at room temperature for 30 mins.

The focused equilibrated strips were run on 2D gel electrophoresis using NuPAGE $4-12 \%$ Bis-Tris precasted gels in a 1x MOPS SDS PAGE running buffer (GE Healthcare sciences, Auckland, New Zealand) containing $1 \mathrm{~mL}$ of NuPAGE antioxidant solution (Invitrogen, Auckland, New Zealand), gels were run at $200 \mathrm{~V}$ for $1 \mathrm{hr}$. The gels were removed from the casts and immersed into a fixative solution (50 $\%$ ethanol $[\mathrm{v} / \mathrm{v}], 2 \%$ orthophosphoric acid $[\mathrm{v} / \mathrm{v}]$, made up to the required volume with distilled water), then incubated at room temperature overnight. Next day the gels were washed with sterile distilled water, for 30 mins 3 times. The gels were stained with Colloidal Coomassie G-250 stain (17 \% ammonium sulfate [w/v], $2 \%$ orthophosphoric acid $[\mathrm{v} / \mathrm{v}], 34 \%$ methanol $[\mathrm{v} / \mathrm{v}]$, made up to the required volume with sterile distilled water) for 2-3 days, after which the gels were washed with sterile distilled water 3-4 times, $1 / 2 \mathrm{hr}$ each time, or until the water was clear. 
Chapter 2: Materials and Methods

\subsubsection{D and 2D gel electrophoresis analysis of Ms/p2196 antisense expressing strain and Ms/pMind protein samples on pH 6-11 isoelectric focusing strips}

The Ms/p2196 antisense expressing strain and Ms/pMind protein samples were also run on $\mathrm{pH}$ 6-11 isoelectric focusing strips. Similar protocol as in 3.5.2 was used except the following changes were made; the strips were rehydrated overnight in rehydration buffer (no protein sample) containing; $7 \mathrm{M}$ urea, $2 \mathrm{M}$ thiourea, $2 \%$ CHAPS, $10 \%$ isopropanol, $5 \%$ glycerol, $1 \%$ IPG buffer (pH 6-11), and $2.5 \%$ DTT. Prior to use, the protein samples were precipitated using Albiochem Proteo Extract protein precipitation kit (GE Healthcare, Auckland, New Zealand) following the manufacturer's instructions. The dried protein pellets were then resuspended in sample buffer similar to the rehydrating buffer mentioned above. 1D gel electrophoresis was run using Ettan IPGphor Isoelectric focusing unit (Amersham Biosciences/GE Healthcare, Auckland, New Zealand), with cup loading following manufacturers protocol.

\subsubsection{Ms/p2196 antisense expressing strain and Ms/pMind 2D gel analysis}

The 2D gels were scanned using Personal Densitometer SI scanner (GE Healthcare, New Zealand). For gel comparison and protein spot selections ImageMaster 2D Platinum 5.0 software (GE Healthcare/Amersham Biosciences, Auckland, New Zealand) was used, prior to the automated spot detection and matching function carried out by the ImageMaster software. Manual specification of spots as landmarks for alignment of the gels was carried out. 


\section{Chapter 2: Materials and Methods}

The selected gel spots were quantified, in term of spot intensity, using ImageJ 1.38x (Wayne Rasband, National Institute of Health, USA). The spot analysis was carried out as follow: control (Ms/pMind) and the antisense expressing strain (Ms/p2196 AS) 2D gels were aligned next to each other. The spots of interest labelled by ImageMaster were selected manually and the intensity of a spot was given in the form of a peak and the area under the peak gave a measurement called "pixel per area" (Figure 2.5.1). The obtained peak values were calculated as a ratio (AS/C when spot was up regulated in antisense and down regulated in control (C/AS when spot was down regulated in antisense but up-regulated in control).

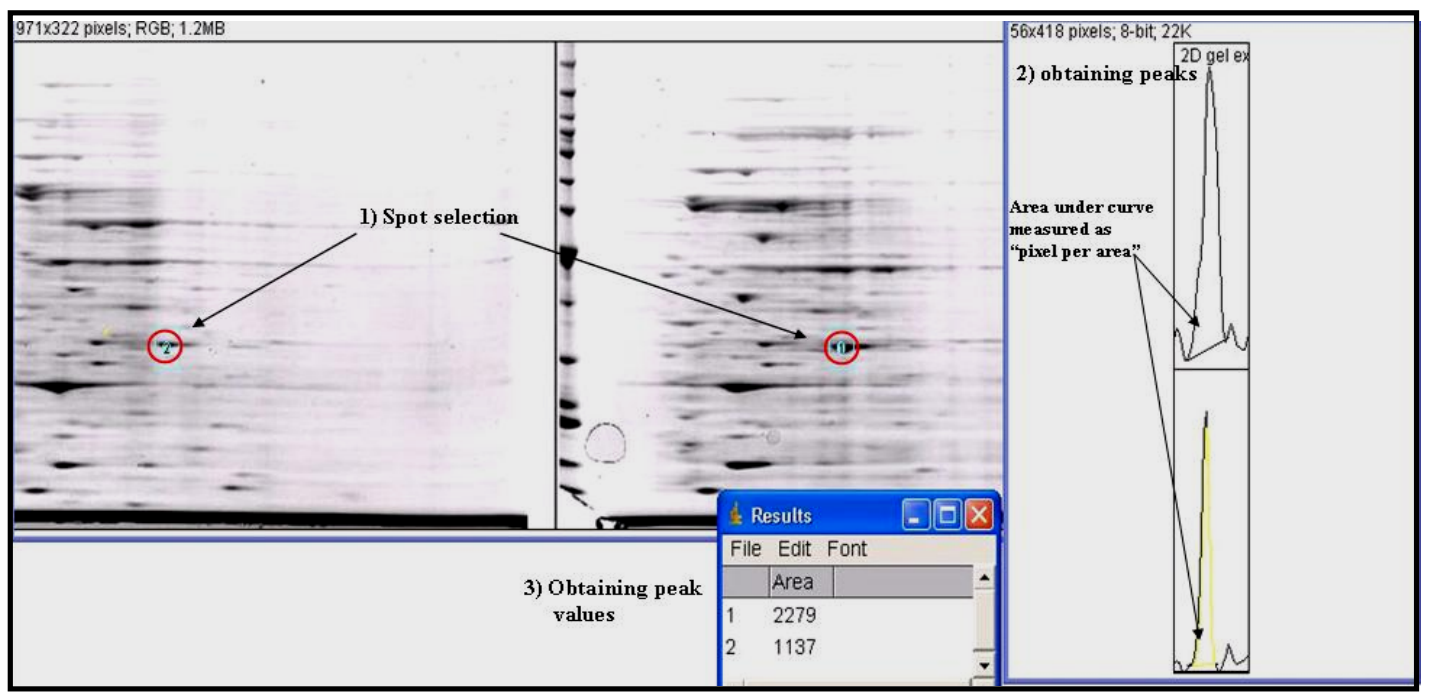

Figure 2.5.1: 2D gel spot intensity quantification using ImageJ software. An example of Ms/pMind (left gel) and Ms/p2196 AS (right gel) spot intensity quantifications. The spots (selected previously by ImageMaster software) were selected manually (1), this selection provides peaks which provide quantitative information (2). The area under the peaks, expressed as "pixel per area" provides values that can be used to determine the relation of the expression fold of a spot on the gel (3). 
Chapter 2: Materials and Methods

Proteins were excised from 2D gels using a OneTouch Plus PDM 1.5 pipette (The gel Company); with TSP $1.5 \mathrm{~mm}$ diameter tips. The excised proteins were placed in a 96 well plate, and overnight trypsin digestion was carried out using an Ettan Digester (Amersham Biosciences. Auckland, New Zealand), trypsin was added at a final concentration of $250 \mathrm{ng}$ in a total volume of $50 \mu \mathrm{L}$ of $20 \mathrm{mM}$ ammonium bicarbonate. The following day, the dried peptides were resuspended in $2 \mu \mathrm{L}$ of matrix ( $\alpha$-cyano-4-hydroxycinnamic [CHCA] dissolved in $0.5 \%$ trifluoroacetic acid [TFA]: acetonitrile $[\mathrm{ACN}][\mathrm{V} / \mathrm{V}]$ in 50:50 dilution), after which they were spotted onto matrix-assisted laser desorption/ionization (MALDI) plate. An external calibration was applied using calibration mixture II (Applied Biosciences, Auckland, New Zealand), $2 \mu \mathrm{L}$ of this was added to $200 \mu \mathrm{L}$ of CHCA matrix solution along with $1 \mu \mathrm{L}$ loaded onto the MALDI plate adjacent to the protein peptide spots (Figure 2.5.2). Using a Voyager DE-Pro (Applied Biosystems, Auckland, New Zealand) MALDI-Time of Flight Mass Spectrum (MALDI-TOF-MS) was employed to produce peptide mass fingerprinting.

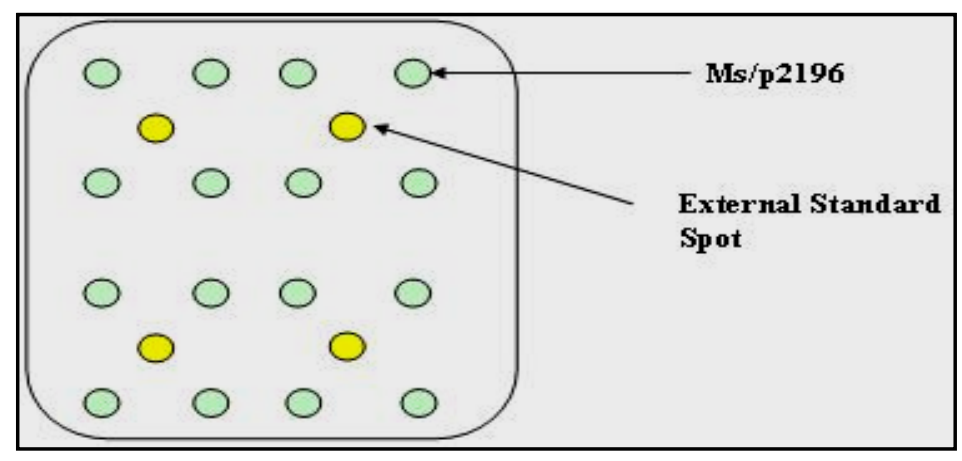

Figure 2.5.2: Example of MALDI plate containing protein peptides of Ms/p2196 AS protein peptides (green) and external calibration sample (yellow). Plate not drawn to scale. 
Chapter 2: Materials and Methods

\subsubsection{Protein database search and protein identifications of Ms/p2196} antisense and Ms/pMind peptide mass fingerprint

For protein database searches, the Matrix Science software was used (http://www.matrixscience.com). The Mascot search engine was selected, and the mass spectrums produced from MALDI-TOF-MS were run in the peptide mass fingerprint database http://www.matrixscience.com/cgi/search form.pl?FORMVER=2\&SEARCH=PMF . Within the Mascot mass fingerprint page, the following selections were made to obtain protein identifications; database: NCBInr, taxonomy: Actinobacteria (class), up to 1 missed cleavage was allowed, the fixed modification used was carbamidomethyl (C), and the peptide tolerance used was 50-100 ppm. Identified proteins were significant if they had a probability greater than $66 \%$ (significant threshold of $\mathrm{P}<0.05)$.

\subsection{Site directed mutagenesis of Mb1389c and MMEG_2196}

\subsubsection{PCR of Mb1389c and MSMEG_2196 flanking region}

The reagent's final concentrations used to amplify the flanking regions of Mb1389c and MSMEG_2196 were similar to that in 2.3.3. The flanking region primers used were; C1/R4 (up-stream fragment) and F2/D2 (down-stream fragment) for Mb1389c; C11/R11 (up-stream fragment) and F13/R16 (down-stream fragment) for MSMEG_2196. 
Chapter 2: Materials and Methods

The flanking region's PCR conditions are similar to that shown in table 2.3.1 and the primers used for the mutagenesis work are listed in table 2.6.1. Primers designed for both Mb1389c and MSMEG_2196 had Pac I digest sites (appropriate to ligate into pUHA778 knock out vector).

\begin{tabular}{|l|l|l|}
\hline $\begin{array}{l}\text { Primer } \\
\text { name }\end{array}$ & $\begin{array}{l}\text { Enzyme } \\
\text { site }\end{array}$ & Sequence \\
\hline Mb1389c C1 & Pac I & CTAGTTAATTAATCGCTCGATGCGGGCATCGAT \\
\hline Mb1389c R4 & & TAACGCGGGTCCGCGGTGTCGTTGCACATCTCGGATGCTA \\
\hline Mb1389c F2 & & AACGACACCGCGGACCCGGCGTTATCTTGA \\
\hline Mb1389c D2 & Pac I & CTAGTTAATTAAAGTTGCGTGCAAGCAGCGG \\
\hline Ms_2196 C11 & Pac I & CTAGTTAATTAAAATGCAGGCACTACACACCGCGG \\
\hline Ms_2196 R11 & & CATGCGAGGCGCAGCACTTGAGCTCAGCCCTGT \\
\hline Ms_2196 F13 & & GGGCTGAGCTCAAGTGCTGCGCCTCGCATGTGAGTGCGG \\
\hline Ms_2196 R16 & Pac I & CTAGTTAATTAATCGTGGCGGACCAGGATCCGT \\
\hline
\end{tabular}

Table 2.6.1: Mb1389c and MSMEG_2196 flanking region and overlap PCR primers. This table lists the primers used to amplify the flanking regions and overlap PCR of Mb1389c and MSMEG_2196. Nucleotides in bold letters represent the enzyme site.

\subsubsection{Mb1389c Overlap PCR}

Overlap PCR, as described previously [56], and outlined in Sambrook and Russell (Volume II 13.36-13.39) [115] was used to join the upstream and downstream fragments of Mb1389c flanking region to create the Mb1389c deletion fragment.

The reagent's final concentrations were $0.2 \mathrm{mM}$ dNTPs, $2.5 \mathrm{mM} \mathrm{MgSO}$, 0.8 $\mu \mathrm{M}$ of each primer $(\mathrm{Mb} 1389 \mathrm{c} \mathrm{C} 1 / \mathrm{D} 2), 1 \mathrm{x}$ PCR reaction buffer, and $0.05 \mathrm{U} / \mu \mathrm{L}$ 
Chapter 2: Materials and Methods

Platinum Taq polymerase. The DNA template was made up of $0.5 \mu \mathrm{g}$ of each flanking region in a $50 \mu \mathrm{L}$ reaction. The $\mathrm{C} 1 / \mathrm{D} 2$ overlap PCR condition for Mb1389c is listed in table 2.6.2.

\begin{tabular}{|l|l|l|}
\hline Steps & $\begin{array}{l}\text { Temperature } \\
\left({ }^{\circ} \mathbf{C}\right)\end{array}$ & $\begin{array}{l}\text { Time } \\
(\mathbf{m i n s})\end{array}$ \\
\hline 1. Heating & 104 & 4.0 \\
\hline 2. Denaturation & 94 & 1.0 \\
\hline 3. Annealing & 45 & 1.0 \\
\hline 4. Extension & 68 & 2.0 \\
\hline 5. Steps 2-4 repeat 40 times & & \\
\hline 6. Extension & 68 & 10 \\
\hline
\end{tabular}

Table 2.6.2: Mb1389c C1/D2 overlap PCR condition. This table lists the PCR condition used to amplify the overlap fragment of Mb1389c.

\subsubsection{MSMEG_2196 overlap PCR}

For the production of the MSMEG_2196 deletion fragment, the initial protocol used was similar to that in 2.6.2; however, after a few attempts with no success a new approach was applied as described previously [23]. The reagent final concentrations were; $0.16 \mathrm{mM}$ dNTPs, the primers Ms_2196 C11 and R16 each at $0.32 \mu \mathrm{M}, 1.6 \mathrm{mM}$ of $\mathrm{MgSO}_{4}, 1 \mathrm{X}$ PCR reaction buffer, $0.04 \mathrm{U} / \mu \mathrm{L}$ of Platinum Taq polymerase, DMSO was also used at $2.5 \%$. 
Chapter 2: Materials and Methods

The template DNA was made up of 0.2 ug of each flanking region in a $31 \mu \mathrm{L}$ total reaction. The PCR protocol involves pausing the $68^{\circ} \mathrm{C}$ extension step (half way through the step), and then adding the up-stream and down-stream primers, in this case the C11/R16 primers, and then continuing the cycle. Table 2.6.3 lists the overlap PCR conditions used for the MSMEG_2196 C11/R16 PCR.

\begin{tabular}{|l|l|l|}
\hline Step & $\begin{array}{l}\text { Temperature } \\
\left({ }^{\circ} \mathbf{C}\right)\end{array}$ & Time (mins) \\
\hline 1. Heating & 94 & 2.0 \\
\hline 2. Denaturation & 94 & 0.30 \\
\hline 3. Annealing & 55 & 0.30 \\
\hline 4. Extension & 68 & 1.0 \\
\hline $\begin{array}{l}\text { 5. Repeat step 2-4 three times. Pause third time } \\
\text { of extension step at 30 secs then add C11/R16 } \\
\text { primer. Continue cycle. }\end{array}$ & & \\
\hline 6. Denaturation & 94 & 0.30 \\
\hline 7. Annealing & 56 & 0.30 \\
\hline 8. Extension & 68 & 5.0 \\
\hline 9. Repeat steps 6-8 x25 & 68 & 10.0 \\
\hline 10. Extension & & \\
\hline
\end{tabular}

Table 2.6.3: PCR conditions used to amplify the MSMEG_2196 overlap fragment. 
Chapter 2: Materials and Methods

\subsubsection{Preparations of Mb1389c and MSMEG_2196 overlap PCR fragments for ligation into pUHA778 knock-out vector.}

Similar protocols to those in steps 2.3.4-2.3.8 were followed for Mb1389c and MSMEG_2196 mutant fragment preparations. One notable change was that the Mb1389c- /MSMEG_2196-overlap PCR fragments were ligated into pGEM-T Easy cloning vector.

\subsection{5 pUHA778 knock-out plasmid preparation}

DNA of the pUHA778 knock out plasmid was supplied from AgResearch (Wallaceville, New Zealand). The isolation of this plasmid was prepared as follow:

$2 \mu \mathrm{L}$ of the pUHA778 DNA was transformed in $250 \mu \mathrm{L}$ of E. coli DH5 $\alpha$ cells resuspended in $0.1 \mathrm{M} \mathrm{CaCl}_{2}$. The cells were plated onto LA-Hyg ${ }_{100}$ plates and incubated at $37{ }^{\circ} \mathrm{C}$ overnight. Colonies from these plates were inoculated for preparation of plasmid as in 2.3.6. To check for the presence of a single Pac I site, the plasmid midipreperations were digested with $0.3 \mathrm{U} / \mu \mathrm{L}$ of Pac I enzyme (concentration at $10 \mathrm{U} / \mu \mathrm{L}$ ), 1 X NEB Buffer 1, and $21.5 \mu \mathrm{L}$ of plasmid in a $30 \mu \mathrm{L}$ total reaction. Roche DNA gel purification kit was used to purify the Pac I-digested pUHA778 plasmid, following the manufacturer's instructions. $5 \mu \mathrm{L}$ of the gel purified pUHA778 plasmid was run on a $1 \%$ low electroendosmosis agarose gel to check for its presence and its quantity. 


\subsubsection{Ligation of Mb1389c- and MSMEG_2196-overlap fragments into pUHA778 and transformation into E. coli DH5a cells}

For the ligation of the Mb1389c and MSMEG_2196 overlap fragments into pUHA778, the ligation reaction contained $2.5 \mu \mathrm{L}$ of Pac I-digested pUHA778 plasmid, $20 \mu \mathrm{L}$ of Pac I-digested Mb1389c-/MSMEG_2196-overlap fragment, 1X ligation buffer 1 (BioLabs, New Zealand), and $0.15 \mathrm{U} / \mu \mathrm{L}$ of T4 DNA ligase in a 60 $\mu \mathrm{L}$ total reaction. The ligation reaction was incubated overnight at $4{ }^{\circ} \mathrm{C}$.

The Mb1389c-/MSMEG_2196-pUHA778-ligated overlap fragments were transformed into E. coli DH5 $\alpha$ cells as described previously in 2.3.5. The transformed cells were plated onto LA-Hyg ${ }_{50} \mathrm{Amp}_{100}$ plates and incubated overnight at $37^{\circ} \mathrm{C}$.

\subsubsection{Mb1389c-/MSMEG_2196-overlap insert check in pUHA778 vector and preparation for electroporation}

Mb1389c-/MSMEG_2196-pUHA778 colonies from the transformed cells in 2.6.6 were inoculated into $100 \mathrm{~mL} \mathrm{LB}-\mathrm{Hyg}_{50} \mathrm{Amp}_{100}$ for preparation of plasmids as in 2.3.6. Pac I enzyme was used, as in 2.3 .7

The Mb1389c/pUHA778 deletion fragment midipreparation was concentrated 6-fold by spinning $320 \mu \mathrm{L}$ in a speed vacuum for $5 \mathrm{hrs}$ at $37^{\circ} \mathrm{C}$ and resuspended in a final volume of $50 \mu \mathrm{L}$. The concentrated DNA was then dialyzed on a nitrocellulose membrane, floating on $60 \mathrm{~mL}$ sterile distilled water for 30 mins. The dialyzed DNA was quantified using a NanoDrop ND-1000 spectrophotometer. The final concentration of the DNA was $0.53 \mu \mathrm{g} / \mu \mathrm{L}$. This DNA was sent to AgResearch, 
Chapter 2: Materials and Methods

Wallaceville, New Zealand PC3 laboratories to be electroporated into M. bovis. For electroporation of Mb1389c/pUHA778 into M. bovis WAg203 see appendix 5.3.

MSMEG_2196/pUHA778 overlap fragment midipreparation was concentrated 6-fold by spinning $200 \mu \mathrm{L}$ in vacuum down to $30 \mu \mathrm{L}$; this concentrated DNA was dialyzed, quantified, and denatured as described above. The DNA final concentration was $1 \mu \mathrm{g} / \mu \mathrm{L}$.

\subsubsection{PCR and Southern blot analysis for single homologous recombination of Mb1389c/pUHA778 in $M$. bovis}

Genomic DNA preparation was carried out on $M$. bovis-pUHA778/Mb1389c heat-killed colonies, obtained from AgResearch, as in 2.3.1 except the pellets were resuspended in $100 \mu \mathrm{L}$ of sterile-distilled water, and run on a $1 \%$ agarose gel to check for DNA quality and quantity. Initially, PCR was used to check for a single homologous recombination. The primer sequences are listed in table 2.6.4. The same PCR set up described in 2.3.2 was used except a $50 \mu \mathrm{L}$ final volume was used and 5 $\mu \mathrm{L}$ of gDNA was added. PCR conditions were set up as in table 2.3.1, except an annealing temperature gradient $\left(45-60^{\circ} \mathrm{C}\right)$ was used.

Southern blot was also used to check for the presence of single and/or double homologous recombinants of Mb1389c in M. bovis. Mb1389c C1/R4 primers (Table 2.6.1) were used to amplify a DNA fragment for use as the hybridization probe. gDNA preparations of heat killed $M$. bovis-pUHA778-Mb1389c colonies were RNAse treated and then digested with Bgl II (BioLabs, Auckland, New Zealand). 
Chapter 2: Materials and Methods

The digestion reaction's final concentrations were; $1 \mathrm{X}$ NEB buffer $3,1.75 \mathrm{U} / \mathrm{ml}$ of Bgl II enzyme, $25 \mu \mathrm{L}$ of gDNA in a total volume of $35 \mu \mathrm{L}$. The digestion reaction was incubated at $37{ }^{\circ} \mathrm{C}$ overnight, and run on a $0.8 \%$ agarose gel at $30 \mathrm{~V}$. For control purposes, wild type Mb1389c, and wild type MSMEG_2196 gDNA were digested with $\mathrm{Bgl}$ II enzyme in the same manner except that $5 \mu \mathrm{L}$ of gDNA was added in a total volume of $10 \mu \mathrm{L}$ digestion reaction. $\alpha-{ }^{32} \mathrm{P}-\mathrm{dCTP}$ (Amersham/GE Healthcare, Auckland, New Zealand) was used as the radiolabel for hybridizations. For a full detailed protocol of Southern blot, refer to appendix 5.4.

The membranes were exposed to film for 2-3 days after which the films were developed and fixed using Kodak processing chemicals for autoradiography films GBX developer and fixer (Sigma-Aldrich, New Zealand).

\begin{tabular}{|l|l|}
\hline Primer name & Sequence \\
\hline DF1 & AAGGCCATCAACGACTACCTG \\
\hline DF2 & AGCGTCACCCCATTGCGC \\
\hline DF3 & AGGCTACATGAGGAAATG \\
\hline DR1 & CGGACCAATGCCTCGGCT \\
\hline DR2 & TACCAGTAGATGCTGGTG \\
\hline DR3 & CATTCGAGACCATATTCG \\
\hline
\end{tabular}

Table 2.6.4: $M$. bovis $\mathrm{p} 1389 \Delta$ primers for analysis of $M$. bovis recombinants. This table lists the primers used in PCR to screen for recombinants of Mb1389c in M. bovis. 
Chapter 2: Materials and Methods

\subsubsection{MSMEG_2196/pUHA778 electroporation into $M$. smegmatis}

1, 5, and $10 \mu \mathrm{L}$ of dialyzed MSMEG_2196/pUHA778 DNA sample, from 2.6.7, was electro-porated into $100 \mu \mathrm{L}$ of $M$. smegmatis $\mathrm{mc}^{2} 155$ competent cells made up to $400 \mu \mathrm{L}$ with $20 \%$ glycerol. The electroporation was carried out at $2.5 \mathrm{kV}$ using a Bio-Rad E. coli pulser electroporator (BioRad, Auckland, New Zealand). $400 \mu \mathrm{L}$ of LB was added to the electroporation mix, and the cells were left to recover at $37{ }^{\circ} \mathrm{C}$ overnight. The next day, the cells were plated onto LA-Hyg $\mathrm{LAmp}_{50}$ plates and incubated at $37{ }^{\circ} \mathrm{C}$ for 1 week. For control purposes, $10 \mu \mathrm{L}$ of empty pUHA778 plasmid was also transformed into M. smegmatis cells in the same manner.

\subsubsection{PCR and Southern blot analysis of $M$. smegmatis/MSMEG_2196- pUHA778 recombinants}

Colonies (2.6.9) were picked and inoculated into $3 \mathrm{~mL}$ of LB broth containing $\mathrm{Hyg}_{50} \mathrm{Amp}_{50}, 0.1 \%$ Tween 80, and were incubated at $37^{\circ} \mathrm{C}$ with shaking at $200 \mathrm{rpm}$ for 2 days after which gDNA was prepared as described in 2.3.1.

To check for the presence of a mutation in MSMEG_2196 knock-out in M. smegmatis, PCR primers used to amplify the flanking region of MSMEG_2196, table 2.6.1, were used; as well as new primers shown in table 2.6.5. The PCR screening was carried out on $M$. smegmatis-pUHA778-MSMEG_2196 gDNA preparations and/or single colonies. PCR conditions used for PCR were set up as in table 2.3.1, and the PCR conditions for single colony screening were conducted as in table 2.3.1. 
Chapter 2: Materials and Methods

Southern blot analysis was also used to check for the presence of single homologous recombination and/or gene knockout. Southern blotting was carried out in the same manner as in 2.6.9, however MSMEG_2196 F4/R4 amplified fragment was used as the probe. Econ I (BioLabs, Auckland, New Zealand) enzyme was used to digest gDNA of $M$. smegmatis/MSMEG_2202-pUHA778, using the protocol described in 2.6.8 except that $1 \mathrm{X}$ NEB buffer 4 (BioLabs, New Zealand) was used. M. smegmatis wild type and $M$. bovis gDNA was used as a positive and negative control, respectively.

\begin{tabular}{|l|l|}
\hline Primer name & sequence \\
\hline Ms_2196 F61 & GAGTCGGCCGCACTCTACGAG \\
\hline Ms_2196 R61 & AAGCGCCAGCAGGTTCAGCAG \\
\hline Ms_2196 F62 & GTGACGACGACCGATCGCGAC \\
\hline Ms-2196R62 & CCTTGACGTAGAGGTCGCCTG \\
\hline Ms_2196 F4 & CTGAAGGCGATCAACGACTACCTG \\
\hline Ms_2196 R4 & CCAGCGGACCAGCGCTTCTGCCGC \\
\hline
\end{tabular}

Table 2.6.5- PCR primers used to check for MSMEG_2196 mutants in M. smegmatis. 


\section{Results}

\subsection{Bioinformatics of cyclic-di-GMP in mycobacteria}

\subsubsection{Cyclic di-GMP protein encoded by the M. tuberculosis, $M$. bovis, and $M$. smegmatis genome}

Since proteins involved in the synthesis of cyclic di-GMP contain GGDEF domain, the $M$. tuberculosis genome was searched for GGDEF domain using TIGRFAMs database (see Materials and Methods section 2.2). Rv1354c, in the genome of M. tuberculosis, was found to be the only gene containing GGDEF-EALGAF domains adjacent to each other (Fig. 3.1.1 A). Rv1354c is annotated as a “conserved hypothetical protein", consisting of 623 amino acids. It has a predicted gene length of 1872 bp. The corresponding ortholog of Rv1354c in M. bovis is Mb1389c and in M. smegmatis is MSMEG_2196, these two had the highest score and sequence alignment to Rv1354c (Table 3.1.1). Refer to appendix 5.5 for gene sequence alignment of the three proteins. Pfam domains structure of Rv1354c was obtained from the annotation database of TIGRFAM (Fig. 3.1.1 A), and a table was given by the database which shows nucleotide boundaries for each of the domain (Fig. 3.1.1 B). 
$\underline{\text { Chapter 3: Results }}$

\begin{tabular}{|l|l|l|l|}
\hline Gene & Score & Amino acid identity & E-value \\
\hline Mb1389c & 9360 & $1872 / 1872(100 \%)$ & 0 \\
\hline MSMEG_2196 & 3776 & $1272 / 1855(68 \%)$ & $7.2 \mathrm{e}-165$ \\
\hline
\end{tabular}

Table 3.1.1: Score and amino acid percentage identity of $M$. bovis and $M$. smegmatis c-di-GMP gene against M. tuberculosis Rv1354c.

\begin{tabular}{|c|c|c|c|}
\hline \multicolumn{4}{|l|}{ (A) } \\
\hline Bili: & GQDEF & 8itl & \\
\hline \multicolumn{4}{|l|}{ (B) } \\
\hline Source & Domain & Start & End \\
\hline PfamA & $\underline{\text { GAF }}$ & 28 & 171 \\
\hline PfamA & GGDEF & 180 & 339 \\
\hline PfamA & $\underline{\text { EAL }}$ & 353 & 595 \\
\hline
\end{tabular}

Figure 3.1.1: Pfam domain search result of GGDEF domain in M. tuberculosis Rv1354c genome. (A) M. tuberculosis Rv1354c domain structure. (B) Boundary of each of the domain showing the start and end of the nucleotide sequence.

\subsubsection{Gene and protein sequences of Mb1389c and MSMEG_2196}

The orthologous of Rv1354c gene sequence in M. bovis and M. smegmatis were derived from TIGRFAM database with 600 bp spanning each end of the sequence. The protein sequences were obtained using the TIGRFAM database. The protein and the DNA sequences are in appendix 5.6. 


\subsection{Altered expression of MSMEG_2196 in M. smegmatis}

\subsubsection{Amplification of MSMEG 2196}

Ms_2196 F1 and R1 primers (Table 2.3.2) were used to amplify MSMEG_2196. The PCR products were run on a $1 \%$ agarose gel (Figure 3.2.1). The amplified product of MSMEG_2196 is between $2-1.6 \mathrm{Kbp}$, this is shown in lane 5. This band was cut and gel purified.

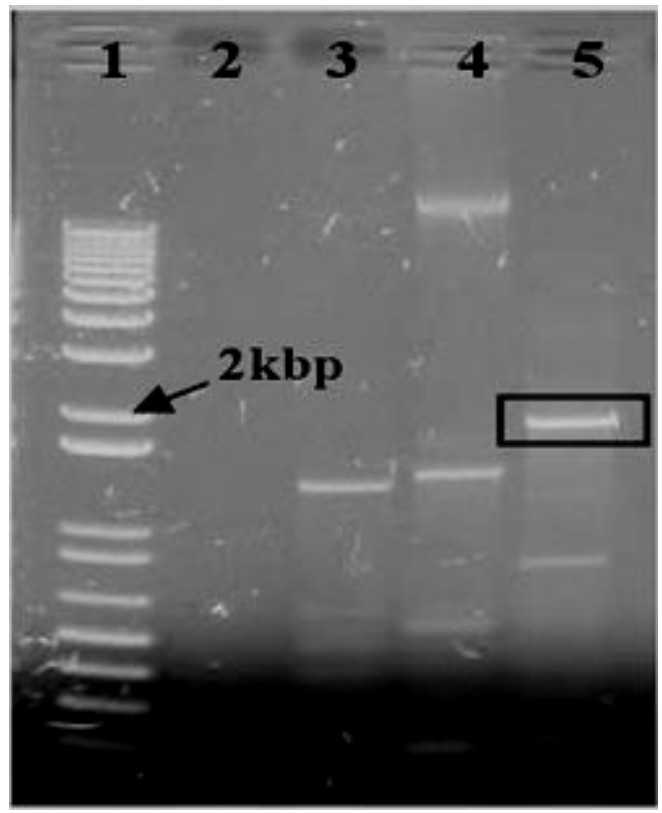

Figure 3.2.1: PCR amplification of MSMEG_2196. Lane 1 contains $1 \mathrm{~Kb}+$ ladder (Invitrogen, New Zealand), lane 2 negative control (no gDNA), and lane 3 positive control (Ms_2196 F3/R3). Lane 4 contains PCR of MSMEG_2196 fragment amplified with Ms_2196 F1/R1 primers no DMSO; this amplification gave unspecific product. Lane 5 contains MSMEG_2196 fragment amplified using Ms_2196 F1/R1 primers 


\subsubsection{Cloning of the MSMEG_2196 gene into pCR2.1 vector}

The amplified product of MSMEG_2196 was ligated into pCR2.1 cloning vector and then transformed into E. coli DH5 $\alpha$ cells. To check for MSMEG_2196 insert in the cloning vector, the colonies obtained from the transformation were digested with Pac I enzyme and run on $1 \%$ agarose gel (Figure 3.2.2). Recombinant plasmids with MSMEG_2196 insert were sequenced to double check for the insert.

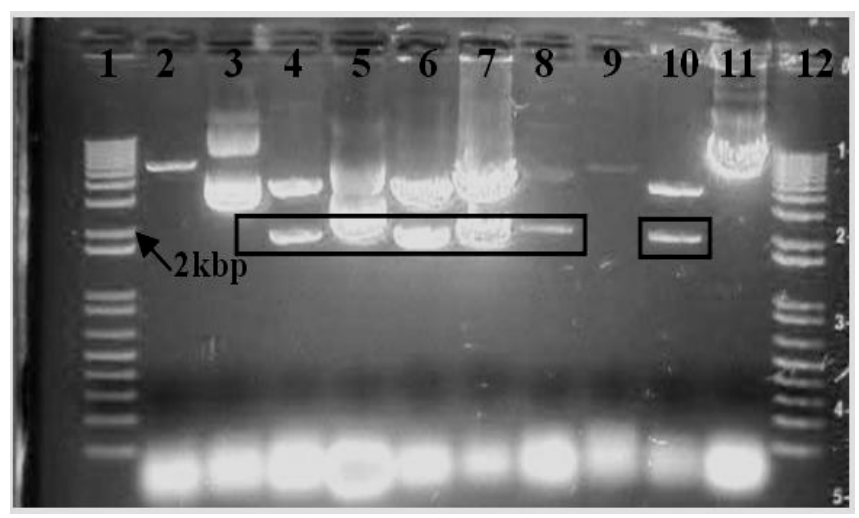

Figure 3.2.2: MSMEG_2196-pCR2.1 Pac I digested plasmids. Lanes 1 and 12 contain $1 \mathrm{~Kb}+$ marker (Invitrogen, New Zealand). Lanes 2-11 contain Pac I digested MSMEG_2196-pCR2.1 recombinant plasmids run on $1 \%$ agarose gel to check for MSMEG_2196 insert. Inserts are present in lanes 4-8 and lane 10 (size of band is between $2-1.6 \mathrm{Kbp}$ ). 


\subsubsection{Ligation of MSMEG_2196 into pMind and check for inserts}

Pac I digested MSMEG_2196 fragment was ligated into Pac I digested and purified pMind plasmid. MSMEG_2196-pMind was transformed into E. coli DH5 $\alpha$ cells. Recombinant plasmids resulting from the transformations were digested with Pac I enzyme, to check for the inserts, and were run on a $1 \%$ agarose gel (Figure $3.2 .3)$.

The expression of a gene in pMind is induced by the influence of tetracycline. In the absence of tetracycline, the regulator tetR represses expression from the tetO operator which results in gene repression. In the presence of tetracycline, repression on the expression of tetO operator (caused by the tetR regulator) is lifted leading to the expression of the gene that is in front of the tetO operator, in this case MSMEG_2196.

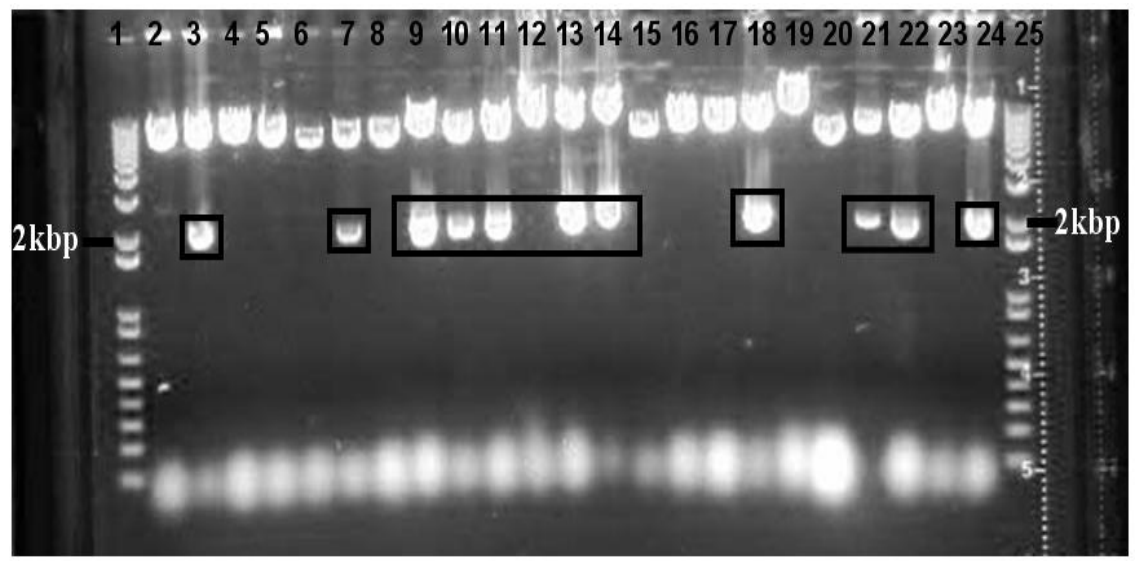

Figure 3.2.3: $1 \%$ agarose gel of MSMEG_2196-pMind recombinant plasmids digested with Pac

I enzyme. Lanes 1 and 25 contains $1 \mathrm{~Kb}+$ marker (Invitrogen, New Zealand). 23 recombinant plasmids of MSMEG_2196-pMind digested with Pac I enzyme were run on agarose gel (lanes 2-24). MSMEG_2196 inserts are in lanes 3, 7, 9-11, 13-14, 18, 21-22, and lane 24, the fragment size is between $2-1.6 \mathrm{Kbp}$. 


\subsubsection{Checking the orientation of the MSMEG_2196 gene in pMind vector}

To check the orientations of the MSMEG_2196 gene (antisense or sense) in pMind, PCR was carried out on single colonies obtained from transformation using primers shown in table 2.3.3.

A sense fragment is produced if PCR products are yielded using TetRO-F and Ms_2196 R1 primers (Figure 3.2.4 A). An antisense fragment will be generated if PCR products are produced using TetRO-F and Ms_2196 F1 primers (Figure 3.2.4 B). In both cases, the PCR product is $\sim 2 \mathrm{kbp}$. 


\section{Chapter 3: Results}

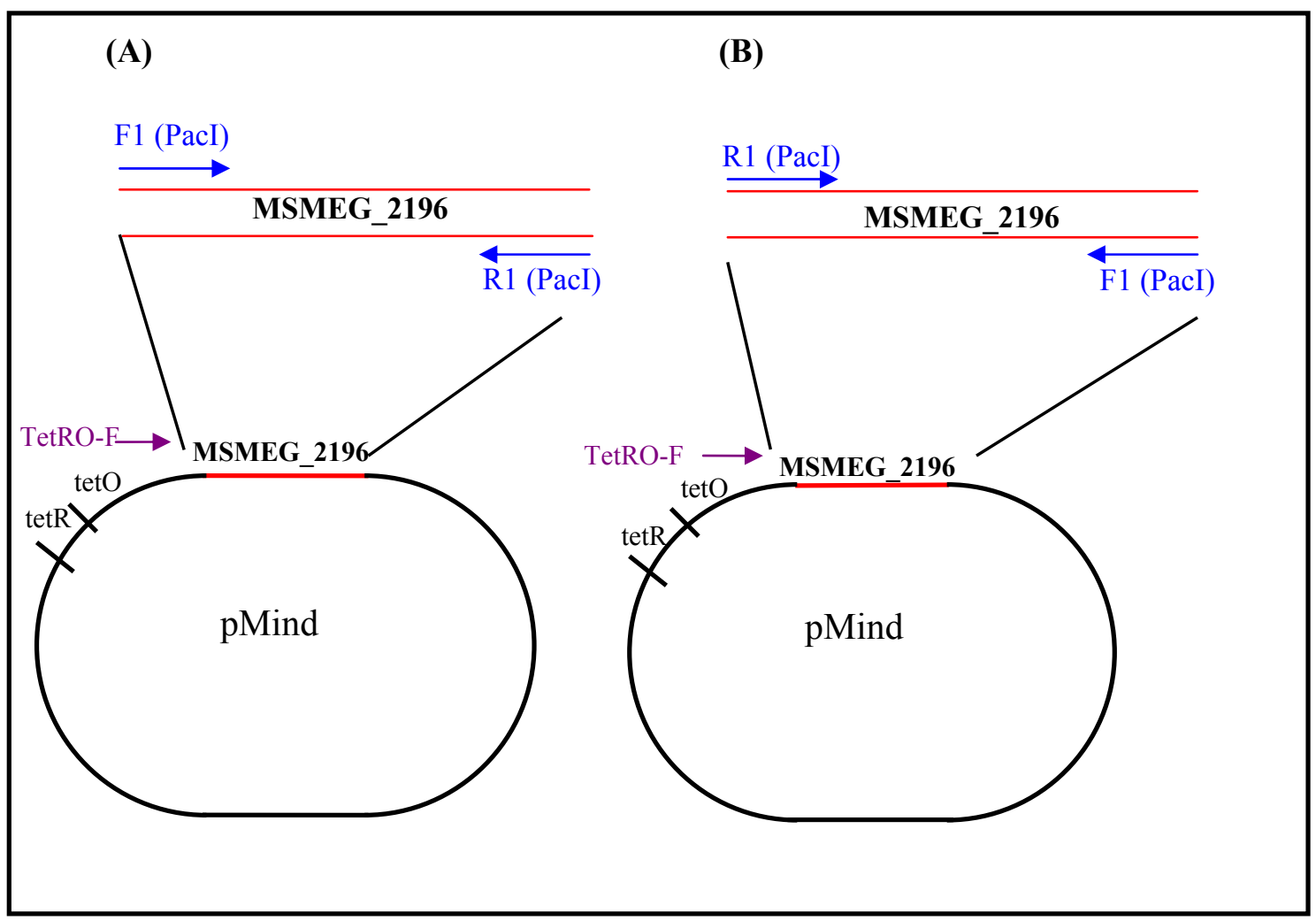

Figure 3.2.4: Possible orientations of MSMEG_2196 in pMind. Depending on the orientation of MSMEG_2196 fragment ligated into the pMind vector. In the above figure (A), a PCR product will be generated if fragment is in the sense orientation using TetRO-F and Ms_2196 R1 primers giving a PCR product of $\sim 2 \mathrm{kbp}$ (in this case there will be no PCR product using Ms_2196 F1 and TetRO-F primers). In figure (B), a PCR product will be generated in the antisense orientation using TetRO-F and Ms_2196 F1 primers once again giving a fragment size of $\sim 2 \mathrm{kbp}$ (using TetRO-F and Ms_2196 $\mathrm{R} 1$ will produce no product in this case).

The forward primer, TetRO-F was used with Ms_2196 F1 or the R1 primers (Table 2.3.2). PCR was run on $1 \%$ agarose gel (Figure 3.2.5, and Table 3.2.1), and from this PCR, MSMEG_2196 antisense and sense samples were determined. 


\section{Chapter 3: Results}

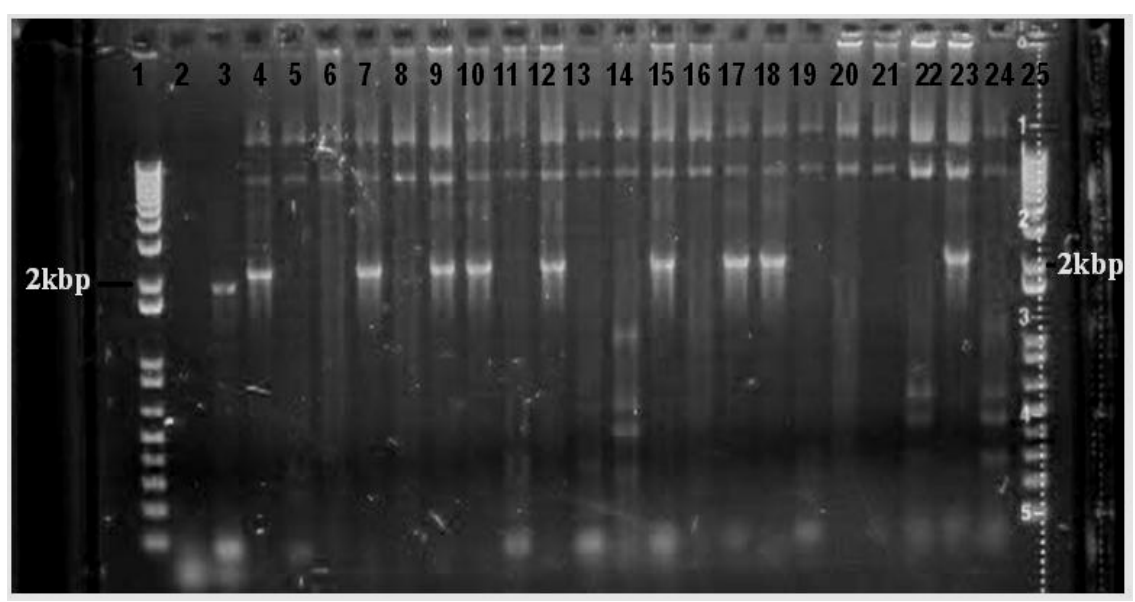

Figure 3.2.5: $1 \%$ agarose gel of antisense and sense PCR of MSMEG_2196-pMind. PCR was carried out on 11 single colonies obtained from MSMEG_2196-pMind transformation. The PCR was run using the TetRO-F primer and Ms_2196 F1 or R1 primers. For each of the 11 single colonies, two PCR reactions were run; one reaction with TetRO-F/Ms_2196 F1 primers, and the other using TetROF/Ms_2196 R1 primers. See table 3.2.1.

After determining the sense and antisense orientation in the MSMEG_2196/pMind transformants, colonies were picked and grown into $50 \mathrm{~mL}$ of LB broth containing $\mathrm{Hyg}_{50} \mathrm{kan}_{50}$ and incubated at $37{ }^{\circ} \mathrm{C}$ for overnight. From these $-80^{\circ} \mathrm{C}$ stocks were made. A sense and antisense sample was chosen for the growth assays experiments. Table 3.2.1 lists all of the amplified products obtained from the MSMEG_2196/pMind PCR conducted to determine the orientation of MSMEG_2196 gene in pMind. 
$\underline{\text { Chapter 3: Results }}$

\begin{tabular}{|c|c|c|c|c|}
\hline $\begin{array}{l}\text { Gel lane } \\
\text { number }\end{array}$ & $\begin{array}{l}\text { Colony } \\
\text { number* }\end{array}$ & Primers & $\begin{array}{l}\text { PCR } \\
\text { products }\end{array}$ & Antisens/Sense \\
\hline 4 & SC1 & TetRO-F/Ms_2196 F1 & Yes & Antisense \\
\hline 5 & SC1 & TetRO-F/Ms_2196 R1 & No & Antisense \\
\hline 6 & $\mathrm{SC} 2$ & TetRO-F/Ms_2196 F1 & No & Sense \\
\hline 7 & $\mathrm{SC} 2$ & TetRO-F/Ms_2196 R1 & Yes & Sense \\
\hline 8 & SC3 & TetRO-F/Ms_2196 F1 & Yes & Senese \\
\hline 9 & SC3 & TetRO-F/Ms_2196 R1 & No & Sense \\
\hline 10 & SC4 & TetRO-F/Ms_2196 F1 & Yes & Antisense \\
\hline 11 & SC4 & TetRO-F/Ms_2196 R1 & No & Antisense \\
\hline 12 & SC5 & TetRO-F/Ms_2196 F1 & Yes & Antisense \\
\hline 13 & SC5 & TetRO-F/Ms_2196 R1 & No & Antisense \\
\hline 14 & SC6 & TetRO-F/Ms_2196 F1 & No & Sense \\
\hline 15 & SC6 & TetRO-F/Ms_2196 R1 & Yes & Sense \\
\hline 16 & SC7 & TetRO-F/Ms_2196 F1 & No & Sense \\
\hline 17 & SC7 & TetRO-F/Ms_2196 R1 & Yes & Sense \\
\hline 18 & SC8 & TetRO-F/Ms_2196 F1 & Yes & Antisense \\
\hline 19 & SC8 & TetRO-F/Ms_2196 R1 & No & Antisense \\
\hline 20 & SC9 & TetRO-F/Ms_2196 F1 & No & PCR did not work \\
\hline 21 & SC9 & TetRO-F/Ms_2196 R1 & No & PCR did not work \\
\hline 22 & SC10 & TetRO-F Ms_2196 F1 & No & Sense \\
\hline 23 & SC10 & TetRO-F/Ms_2196 R1 & Yes & Sense \\
\hline 24 & SC11 & TetRO-F Ms_2196 F1 & No & PCR did not work \\
\hline
\end{tabular}

Table 3.2.1: Antisense and sense PCR results of MSMEG_2196-pMind. The results of the $1 \%$ agarose gel shown in figure 3.2.5. It demonstrates which of the samples, run on the gel, contained the gene in the sense or antisense orientation.

$* \mathrm{SC}=$ single colony 


\subsection{Growth assays of MSMEG_2196-pMind antisense/sense culture}

\subsubsection{Nutrient rich growth assay of MSMEG_2196-pMind antisense/sense}

The four strains: M. Smeg WT, Ms/pMind, Ms/p2196 S, and Ms/p2196 AS were grown in LB broth. This nutrient rich assay was used as a control to compare the growth of the four strains to that of the nutrient limited-growth assays and to see if either antisense and/or sense strain in rich media alone could have any effects on growth under nutrient-rich conditions. The experiment was conducted in triplicate, with $0 \mathrm{ng} / \mathrm{mL}$, and $40 \mathrm{ng} / \mathrm{mL}$ of $\mathrm{Tc}$. $\mathrm{OD}_{600}$ absorbance readings were taken every 90 mins for the first $11 \mathrm{hrs}$ and then every $3 \mathrm{hrs}$ the next day. The growth curves in the presence of $0 \mathrm{ng} / \mathrm{mL}$ and $40 \mathrm{ng} / \mathrm{mL}$ of Tc are shown in figure 3.3.1 A and $\mathrm{B}$, and specific growth rates and doubling times were also calculated and these are shown in table 3.3.1.

Standard deviation of the mean, also known as standard error, was calculated using Microsoft Excel. The mean of the triplicates was calculated and from the mean, standard deviation was obtained using Excel. The standard error was calculated by dividing standard deviation by the square root of the number of measurements, in this

case by $\sqrt{3}$. From the growth curve, the specific growth rates and doubling times clearly show that the growth of all four cultures was not affected in either the absence or presence of Tc in the rich LB broth media. 


\section{Chapter 3: Results}

(A)

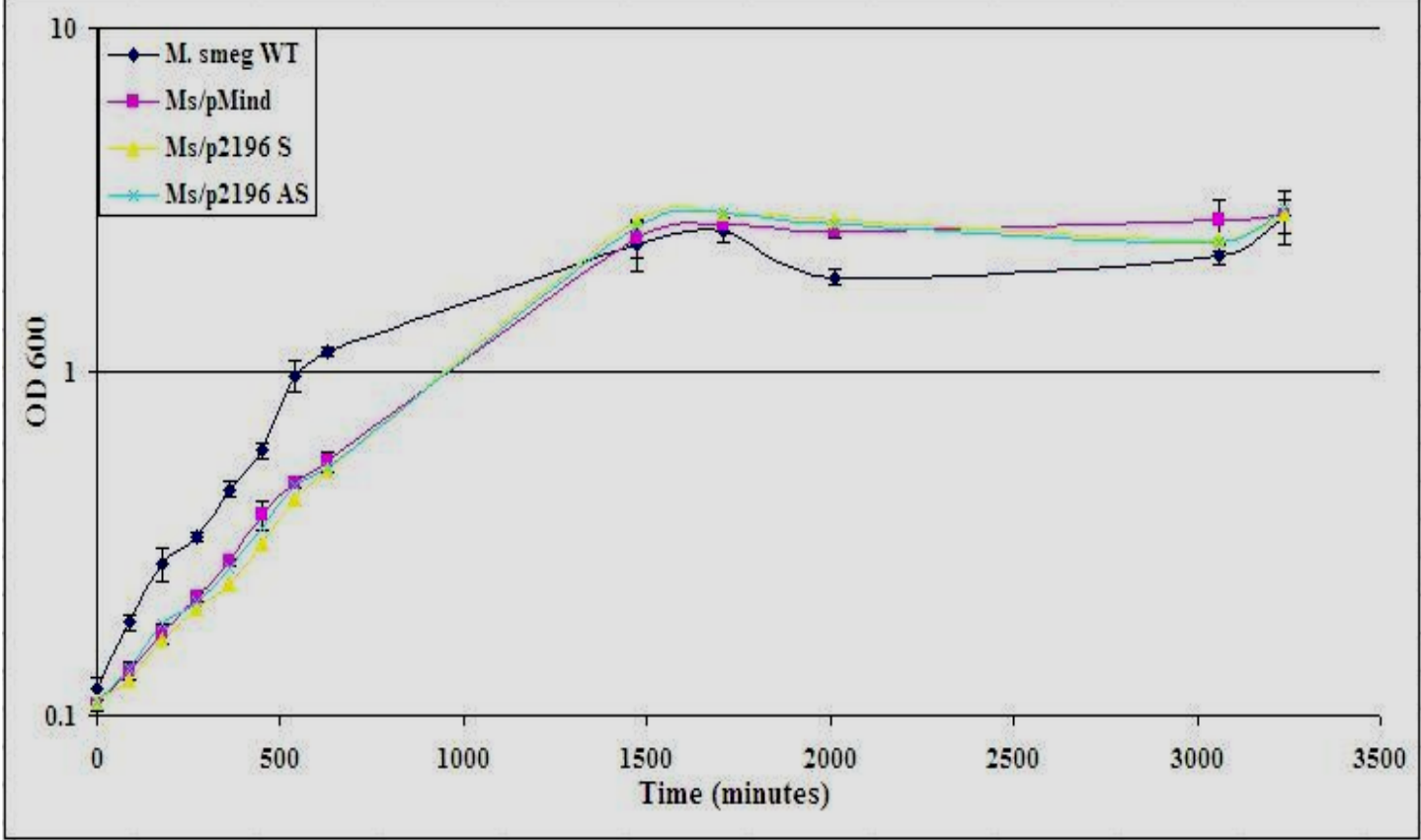

(B)

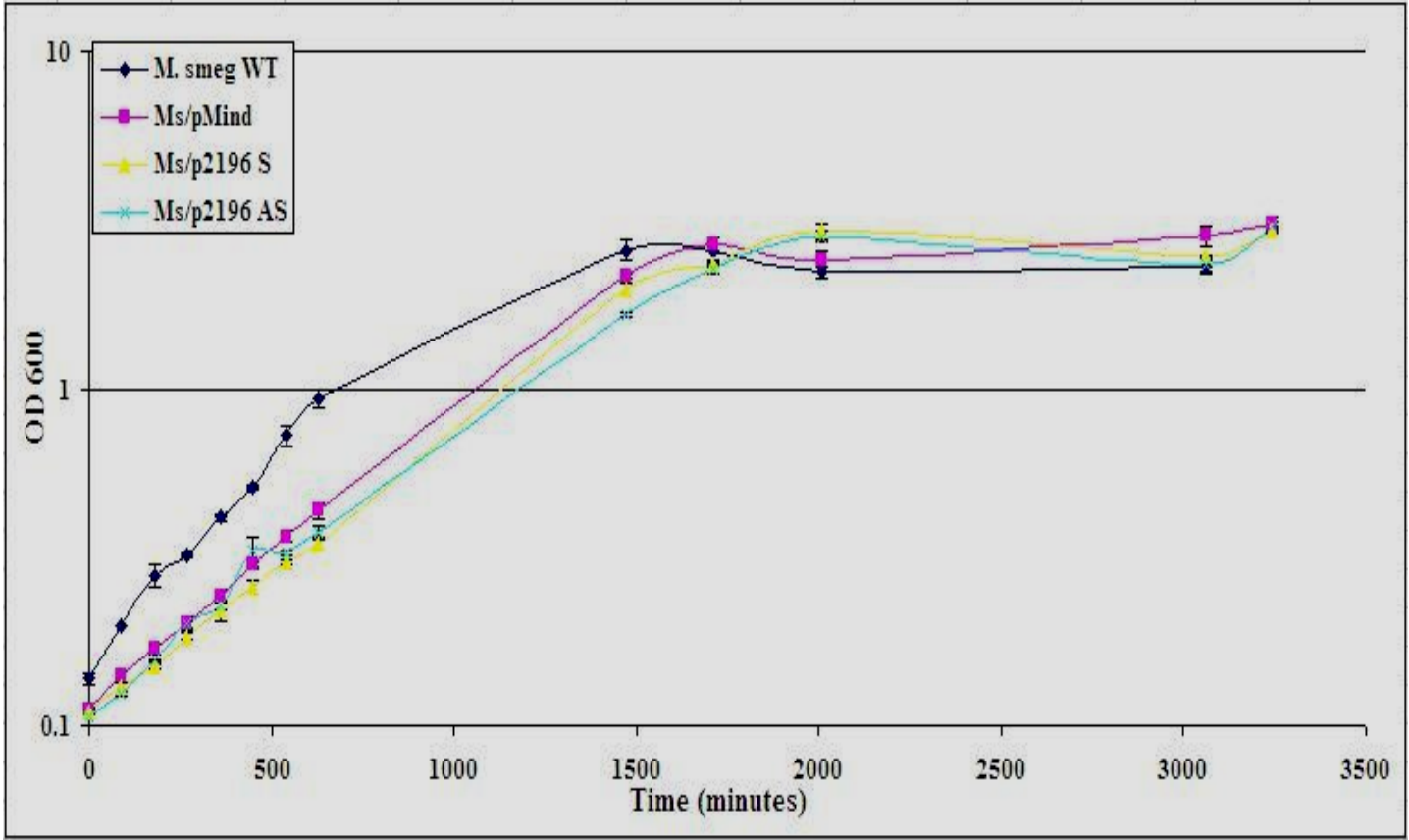

Figure 3.3.1: Growth curve of M. smegmatis WT, Ms/pMind, Ms/p2196 sense, and Ms/p2196

antisense grown in LB broth rich media. The four strains were grown in LB broth containing either $0 \mathrm{ng} / \mathrm{mL}$ (A), or $40 \mathrm{ng} / \mathrm{mL}$ (B) of Tc. On the first day, the $\mathrm{OD}_{600}$ readings of the assay were taken every 90 mins, then every 3 hrs there after. The results were plotted on a semi-log graph. 
$\underline{\text { Chapter 3: Results }}$

\begin{tabular}{|l|l|l|l|}
\hline $\begin{array}{l}\text { M. smegmatis } \\
\text { strian }\end{array}$ & Te & $\begin{array}{l}\text { Log-phase specific growth } \\
\text { rate }\left(\mathbf{m i n}^{-1}\right)\end{array}$ & $\begin{array}{l}\text { Doubling time } \\
(\mathbf{m i n s})\end{array}$ \\
\hline M. smeg WT & - & $0.003192( \pm 0.0000194)$ & $217( \pm 1.32)$ \\
\hline M. smeg WT & + & $0.002610( \pm 0.0000363)$ & $266( \pm 3.70)$ \\
\hline Ms/pMind & - & $0.002447( \pm 0.0004650)$ & $283( \pm 5.26)$ \\
\hline Ms/pMind & + & $0.002107( \pm 0.0000430)$ & $329( \pm 6.71)$ \\
\hline Ms/p2196 S & - & $0.002503( \pm 0.0000844)$ & $277( \pm 9.34)$ \\
\hline Ms/p2196 S & + & $0.001875( \pm 0.0000474)$ & $370( \pm 9.34)$ \\
\hline Ms/p2196 AS & - & $0.002620( \pm 0.0000589)$ & $265( \pm 5.95)$ \\
\hline Ms/p2196 AS & + & $0.002698( \pm 0.0000471)$ & $257( \pm 4.48)$ \\
\hline
\end{tabular}

Table 3.3.1: Specific growth rates and doubling times for nutrient rich (LB broth) growth assay. This table lists the growth rates and doubling time of the four cultures grown in the LB broth rich media in the presence and absence of Tc.

\subsubsection{Carbon limited growth assay of MSMEG_2196-pMind antisense/sense}

M. smegmatis WT, Ms/pMind, Ms/p2196 S, and Ms/2196 AS were grown in Hartman-de Bont $(\mathrm{HdeB})$ carbon limited $\left(\mathrm{C}^{-}\right)$media supplemented with $0.08 \%$ ( $\mathrm{vol} / \mathrm{vol}$ ) glycerol. Tc was added to the culture at concentrations of $0 \mathrm{ng} / \mathrm{mL}$ and 40 $\mathrm{ng} / \mathrm{mL}$. The cultures were grown in triplicate, and as with the rich media, the $\mathrm{OD}_{600}$ readings were taken every 90 mins for the first $11 \mathrm{hrs}$ then $3 \mathrm{hrs}$ daily for 2 days. Standard error was calculated as described in 3.3.1. Both cultures (Tc-, Tc+) grown in the carbon-limited media had no growth effect in the exponential- and stationaryphase (Figure 3.3.2). However, cultures grown in the presence of Tc grew at a slower rate. Specific growth rates and doubling times between the four cultures did not vary significantly, i.e. no difference outside the standard error values (Table 3.3.2). 


\section{Chapter 3: Results}

(A)

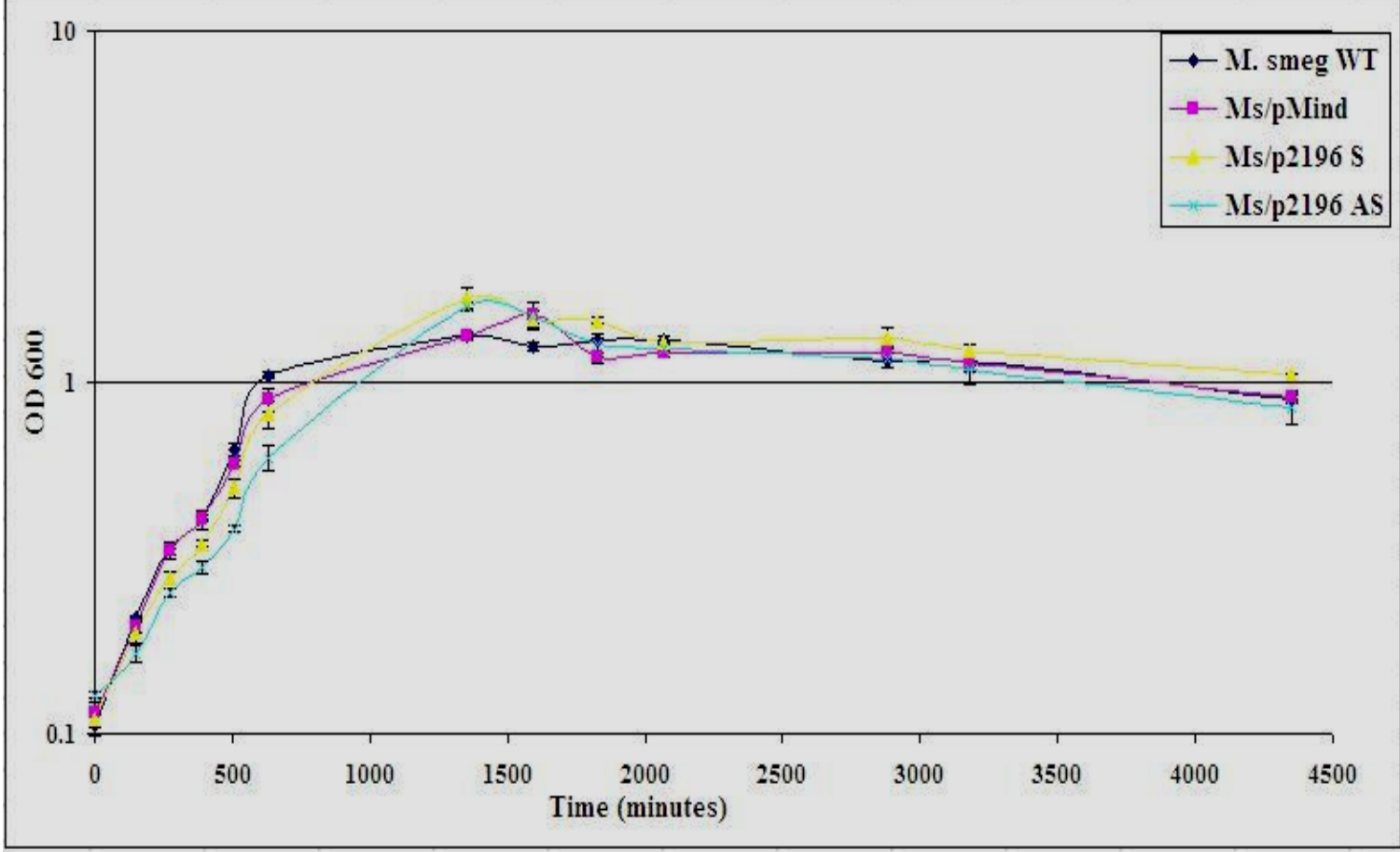

(B)

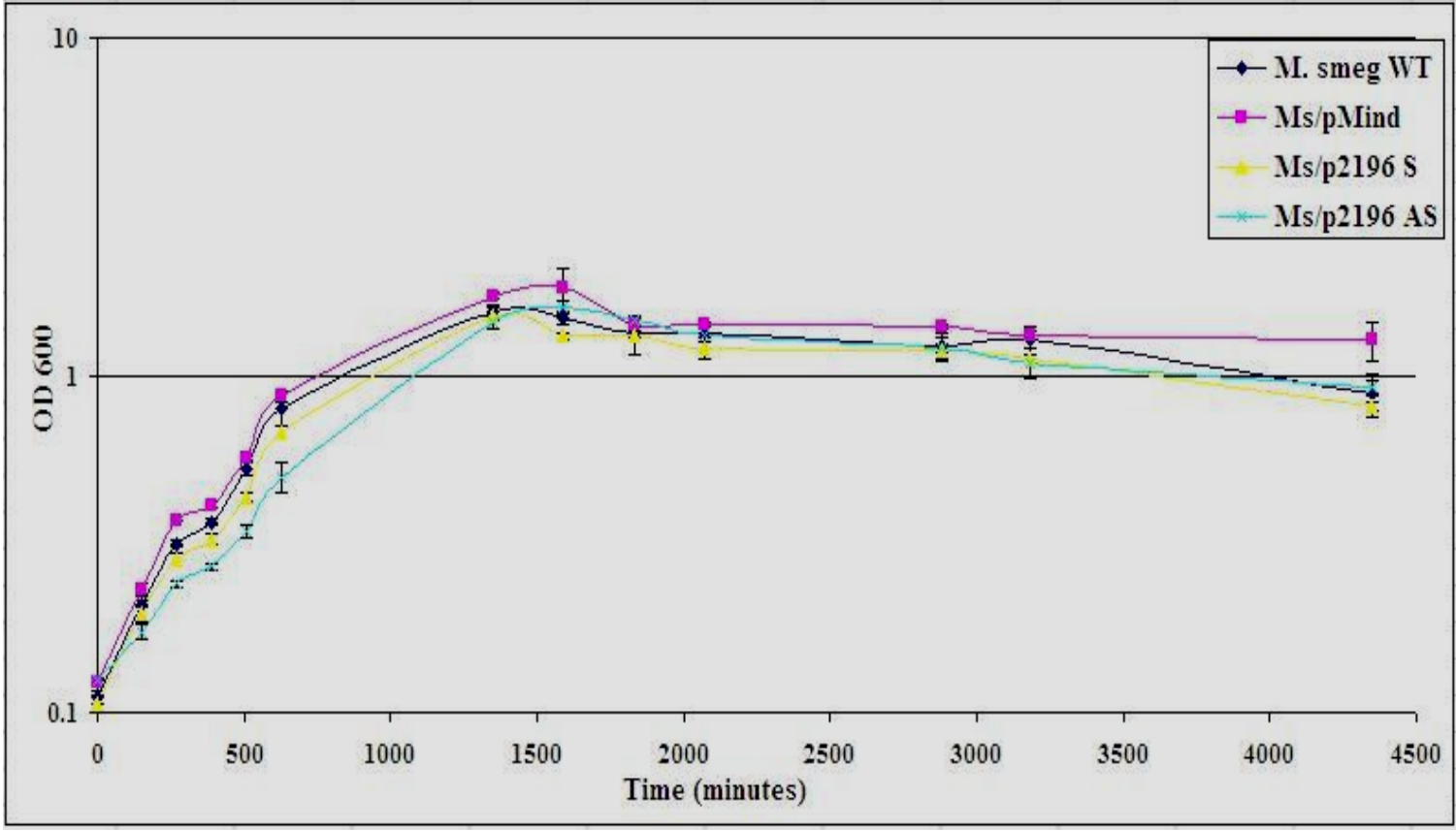

Figure 3.3.2: Growth curve of $M$. smegmatis WT, Ms/pMind, Ms/p2196 S, and Ms/p2196 AS grown in HdeB $\mathrm{C}^{-}$limited media. This graph illustrates the four strains grown in $\mathrm{HdeB} \mathrm{C}^{-}$limited media containing either $0 \mathrm{ng} / \mathrm{mL}$ (A), or $40 \mathrm{ng} / \mathrm{mL}$ (B) of Tc. On the first day, the $\mathrm{OD}_{600}$ readings of the assays were taken every $90 \mathrm{mins}$, then every $3 \mathrm{hrs}$ there after, up to 2 days. Overall, outside of standard errors, there was no significant difference (outside of standard errors) or growth change between the four cultures grown in either the presence or absence of Tc. The results were plotted on a semi-log graph. 
Chapter 3: Results

\begin{tabular}{|l|l|l|l|}
\hline $\begin{array}{l}\text { M. smegmatis } \\
\text { strain }\end{array}$ & Tc & $\begin{array}{l}\text { Log-phase specific growth } \\
\text { rate }\left(\mathbf{m i n}^{-1}\right)\end{array}$ & $\begin{array}{l}\text { Doubling time } \\
(\mathbf{m i n s})\end{array}$ \\
\hline M. smeg WT & - & $0.002810( \pm 0.000303)$ & $247( \pm 26.56)$ \\
\hline M. smeg WT & + & $0.002655( \pm 0.000220)$ & $261( \pm 21.62)$ \\
\hline Ms/pMind & - & $0.002962( \pm 0.000049)$ & $234( \pm 3.87)$ \\
\hline Ms/pMind & + & $0.002525( \pm 0.000055)$ & $274( \pm 5.97)$ \\
\hline Ms/p2196 S & - & $0.002708( \pm 0.000182)$ & $256( \pm 17.20)$ \\
\hline Ms/p2196 S & + & $0.002250( \pm 0.000090)$ & $308( \pm 12.32)$ \\
\hline Ms/p2196 AS & - & $0.002238( \pm 0.000054)$ & $310( \pm 7.48)$ \\
\hline Ms/p2196 AS & + & $0.001916( \pm 0.000046)$ & $362( \pm 8.69)$ \\
\hline
\end{tabular}

Table 3.3.2: $\quad$ Specific growth rates and doubling times for $\mathrm{C}^{-}$limited growth assay in HdeB media. This table lists the growth rates and doubling time of the four cultures grown in the carbon limited HdeB media in the presence/absence of Tc. Overall, there are no significant differences (outside of standard errors) in either the growth rate or doubling time in all four cultures.

\subsubsection{Oxygen limited growth assay of MSMEG_2196-pMind antisense/sense}

M. smegmatis WT, Ms/pMind, Ms/p2196 S, and Ms/p2196 AS were grown in $150 \mathrm{~mL}$ of HdeB oxygen $\left(\mathrm{O}^{-}\right)$limited media (see 2.4.1). The four cultures were grown in triplicate with $0 \mathrm{ng} / \mathrm{mL}$ and $40 \mathrm{ng} / \mathrm{mL}$ of Tc. $\mathrm{OD}_{600}$ absorbance readings were taken every 90 mins for the first 10 hrs then every 3 hrs daily for 2 days (Figure 3.3.3). Standard errors were calculated as described in 3.3.1. The specific growth rates and doubling times of the oxygen limited assay are shown in table 3.3.3. In the presence of Tc, the growth of Ms/p2196 antisense was slightly affected after $3 \mathrm{hr}$ of growth (Figure 3.3.3). This slight change is not significant as shown by the specific growth rate and doubling time (not significant outside of standard errors) calculated (Table 3.3.3). 


\section{Chapter 3: Results}

(A)

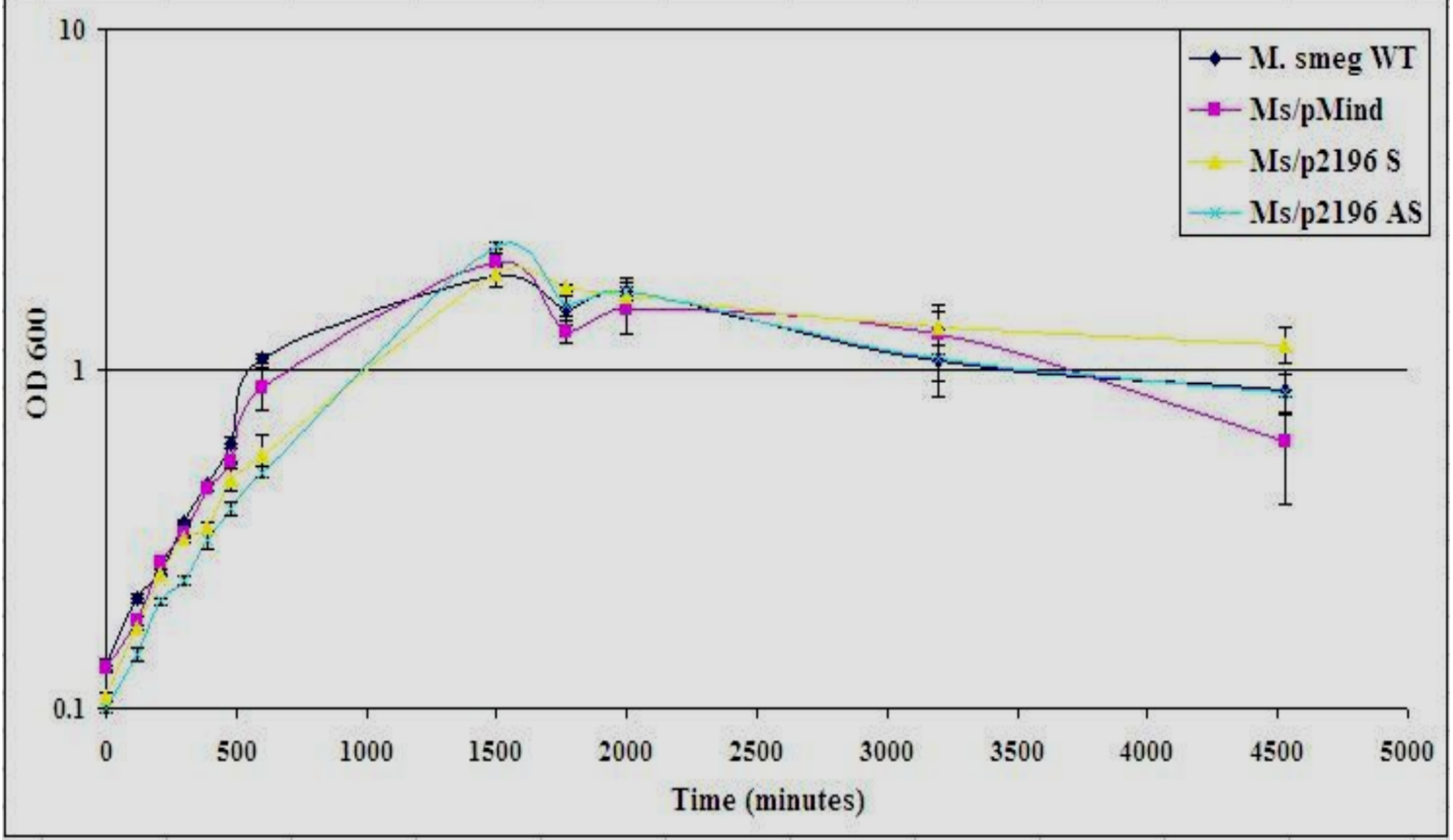

(B)

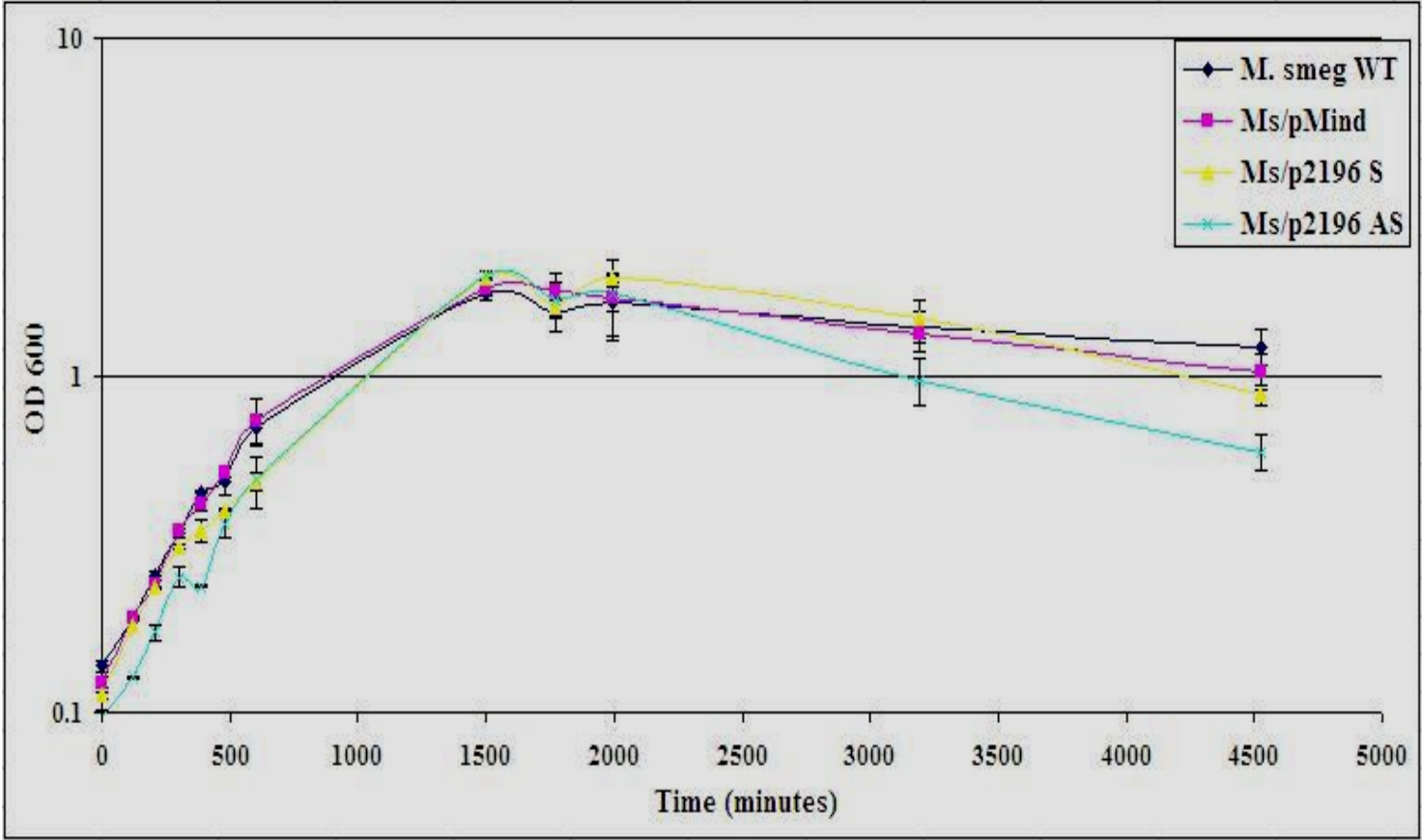

Figure 3.3.3: Growth curve of $M$. smegmatis WT, Ms/pMind, Ms/p2196 S, and Ms/p2196 AS grown in $\mathrm{HdeB} \mathrm{O}^{-}$limited media. This graph shows the four strains grown in $\mathrm{HdeB}^{-}$limited media containing either $0 \mathrm{ng} / \mathrm{mL}$ (A), or $40 \mathrm{ng} / \mathrm{mL}$ (B) of Tc. On the first day, the $\mathrm{OD}_{600}$ readings of the assays were taken every $90 \mathrm{mins}$, then every $3 \mathrm{hrs}$ there after, up to 2 days. The Ms/p2196 antisense strain, in the presence of $\mathrm{Tc}$, grew slightly at a lower rate at stationary-phase compared to the three other strains. However, from the calculated specific growth rates and doubling times this slight change is not significant. These results were plotted on a semi-log graph. 
$\underline{\text { Chapter 3: Results }}$

\begin{tabular}{|l|l|l|l|}
\hline $\begin{array}{l}\text { M. smegmatis } \\
\text { strain }\end{array}$ & Tc & $\begin{array}{l}\text { Log-phase specific growth } \\
\text { rate }\left(\mathbf{m i n}^{-1}\right)\end{array}$ & $\begin{array}{l}\text { Doubling time } \\
(\mathbf{m i n s})\end{array}$ \\
\hline M. smeg WT & - & $0.003522( \pm 0.000604)$ & $197( \pm 33.74)$ \\
\hline M. smeg WT & + & $0.003860( \pm 0.000721)$ & $180( \pm 33.60)$ \\
\hline Ms/pMind & - & $0.004055( \pm 0.000710)$ & $171( \pm 29.95)$ \\
\hline Ms/pMind & + & $0.003406( \pm 0.001012)$ & $203( \pm 60.24)$ \\
\hline Ms/p2196 S & - & $0.003001( \pm 0.001235)$ & $231( \pm 95.06)$ \\
\hline Ms/p2196 S & + & $0.002830( \pm 0.001041)$ & $245( \pm 90.07)$ \\
\hline Ms/p2196 AS & - & $0.003501( \pm 0.000532)$ & $198( \pm 30.09)$ \\
\hline Ms/p2196 AS & + & $0.003076( \pm 0.000636)$ & $226( \pm 54.96)$ \\
\hline
\end{tabular}

Table 3.3.3: Specific growth rates and doubling times for $\mathrm{O}^{-}$limited growth assay in HdeB media. This table lists the growth rates and doubling times of the four cultures grown in the $\mathrm{O}^{-}$ limited HdeB media in the presence/absence of Tc. Overall, there was no significant difference (outside of standard errors) in either the growth rate or doubling times in all four cultures.

\subsubsection{Phosphorous limited growth assay of MSMEG_2196-pMind antisense/sense culture.}

The four cultures, M. smegmatis WT, Ms/pMind, Ms/p2196 S, and Ms/p2196 AS, were grown in HdeB phosphorous limited $\left(\mathrm{P}^{-}\right)$media. The phosphorous was reduced $100 \mathrm{mM}$ to $0.1 \mathrm{mM}$. Glycerol was added at a final concentration of $0.2 \%$. Standard errors of the growth curve were generated as described in 3.3.1. The four cultures were grown in triplicate with $0 \mathrm{ng} / \mathrm{mL}$ and $40 \mathrm{ng} / \mathrm{mL}$ of $\mathrm{TC}$. $\mathrm{OD}_{600}$ absorbance readings were taken every 90 mins for the first 10 hrs then every 3 hrs the next day. 


\section{Chapter 3: Results}

The four cultures grown in the phosphorous-limited media showed no significant growth effect difference (outside of standard errors) in either the presence or absence of Tc (Figure 3.3.4). One notable difference is that at exponential-phase (in the presence of $\mathrm{Tc}$ ), all four cultures grew at a slower rate (these cultures have high doubling times compared to doubling times of culture with no Tc). This growth effect could be due to the phosphorous-limited media, compared to the four strains grown in carbon-, or oxygen-limited media. This slight change observed is not significant enough (outside of standard errors) to conclude that the over- and/or down-expression of MSMEG_2196 in M. smegmatis are affected because the changes seen in the growth behaviour is observed in all four cultures. 


\section{Chapter 3: Results}

(A)

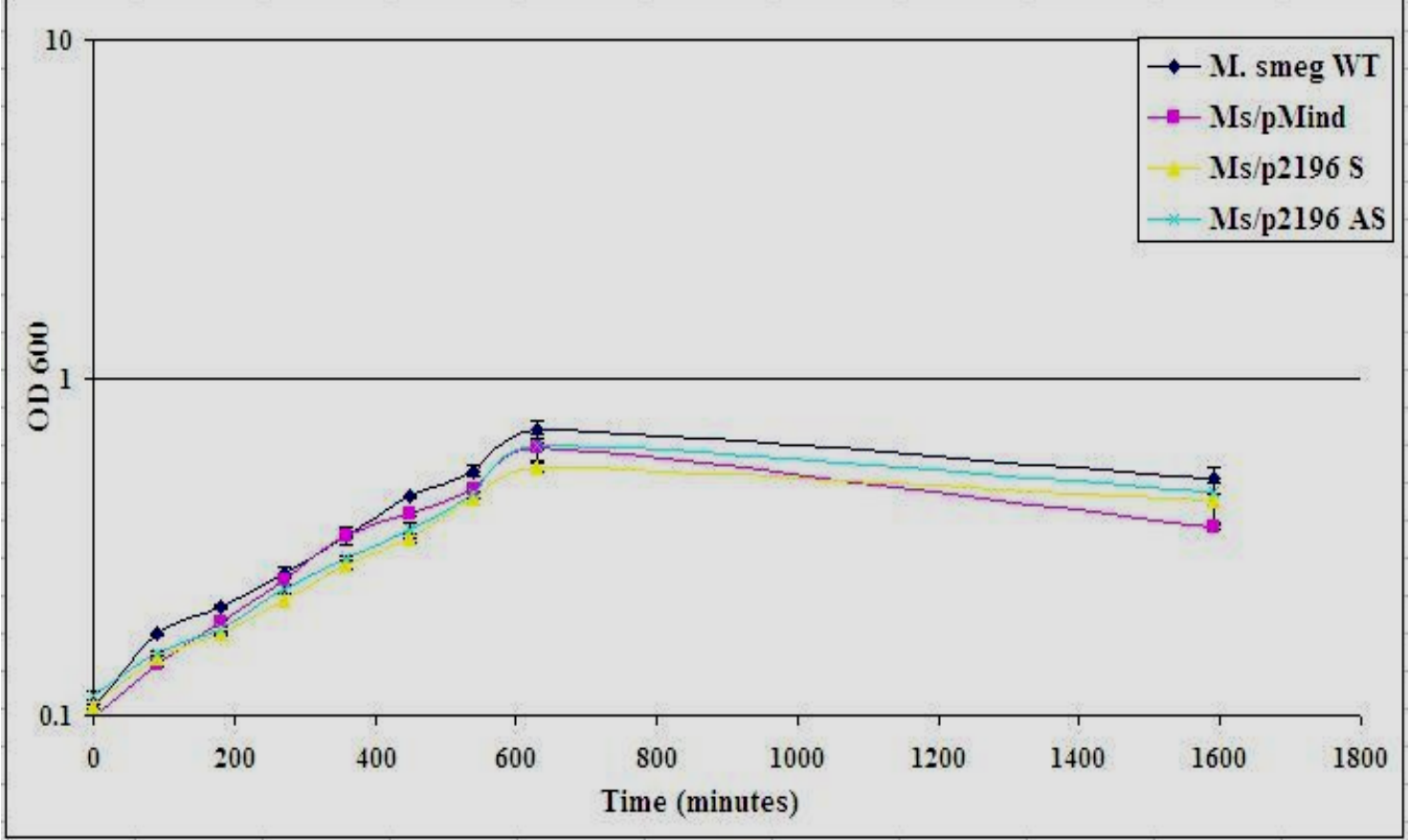

(B)

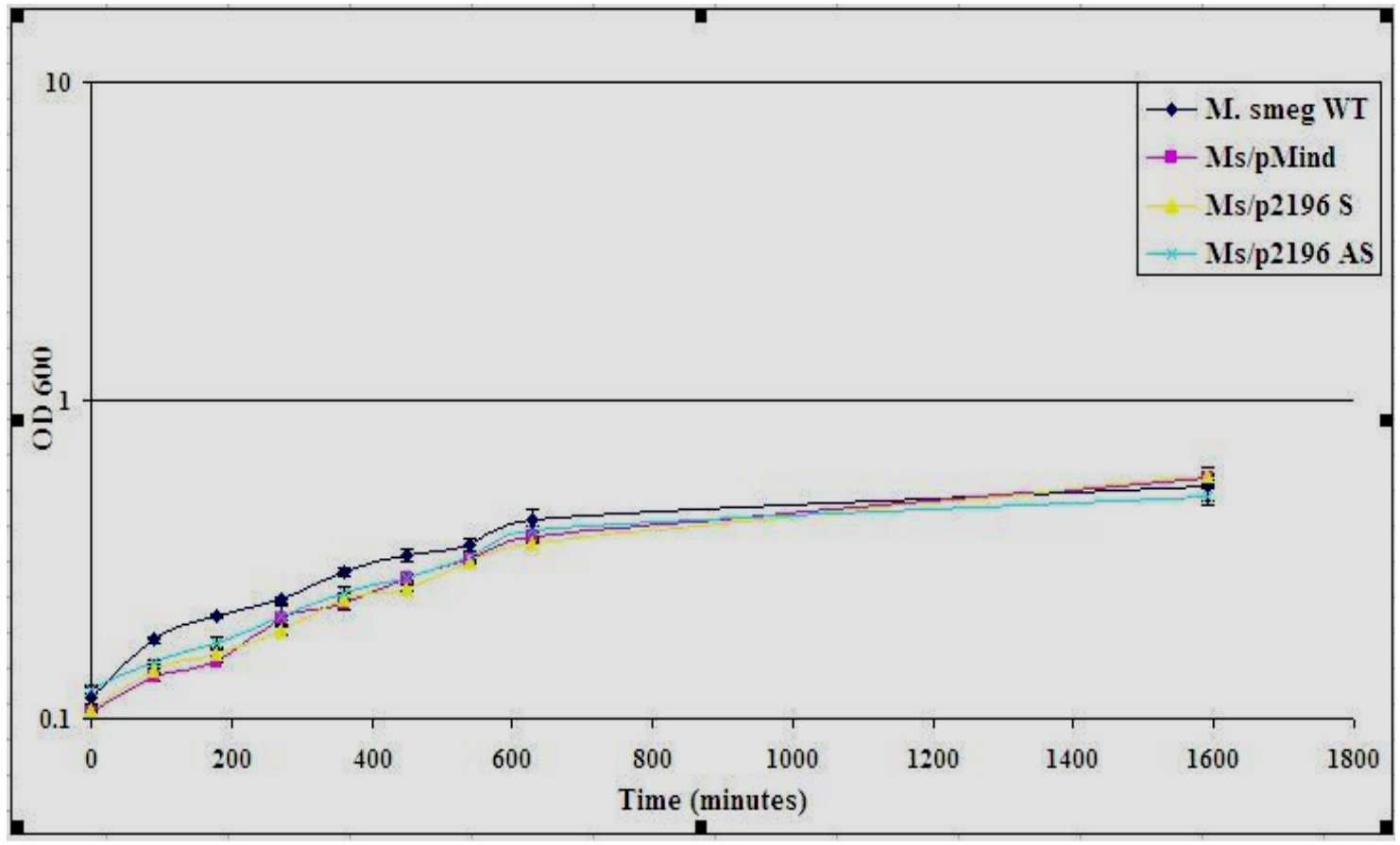

Figure 3.3.4: Growth curve of M. smegmatis WT, Ms/pMind, Ms/p2196 S, and Ms/p2196 AS grown in HdeB $\mathbf{P}^{-}$limited media. This graph illustrates the growth of the four strains grown in HdeB $\mathrm{P}^{-}$limited media containing either $0 \mathrm{ng} / \mathrm{mL}$ (A), or $40 \mathrm{ng} / \mathrm{mL}$ (B) of Tc. On the first day, the $\mathrm{OD}_{600}$ readings of the assays were taken every $90 \mathrm{mins}$, then every $3 \mathrm{hrs}$ the next day. Overall, outside of standard errors, there is no significant difference or changes between the growth of the four cultures in either the presence and absence of Tc. The results were plotted on a semi-log graph. 
Chapter 3: Results

\begin{tabular}{|l|l|l|l|}
\hline $\begin{array}{l}\text { M. smegmatis } \\
\text { strain }\end{array}$ & Tc & $\begin{array}{l}\text { Log-phase specific growth } \\
\text { rate }\left(\mathbf{m i n}^{-1}\right)\end{array}$ & $\begin{array}{l}\text { Doubling time } \\
(\mathbf{m i n s})\end{array}$ \\
\hline M. smeg WT & - & $0.002462( \pm 0.00023670)$ & $281( \pm 30.48)$ \\
\hline M. smeg WT & + & $0.001471( \pm 0.00021034)$ & $471( \pm 67.38)$ \\
\hline Ms/pMind & - & $0.002542( \pm 0.00043487)$ & $273( \pm 46.67)$ \\
\hline Ms/pMind & + & $0.001662( \pm 0.00024326)$ & $417( \pm 61.03)$ \\
\hline Ms/p2196 S & - & $0.002447( \pm 0.00006200)$ & $283( \pm 7.17)$ \\
\hline Ms/p2196 S & + & $0.001713( \pm 0.00011656)$ & $405( \pm 47.55)$ \\
\hline Ms/p2196 AS & - & $0.002409( \pm 0.00001382)$ & $288( \pm 1.65)$ \\
\hline Ms/p2196 AS & + & $0.001639( \pm 0.00014222)$ & $423( \pm 36.72)$ \\
\hline
\end{tabular}

Table 3.3.4: Specific growth rates and doubling times for $\mathrm{P}^{-}$limited growth assay in HdeB media. Growth rates and doubling time of the four cultures grown in the $\mathrm{P}^{-}$limited HdeB media in the presence/absence of Tc. Overall, the four cultures grown in phosphorous-limited media grew at a slower rate compared to carbon-, or oxygen-limited growth assays. The four strains grown in the presence of Tc grew at a slower rate (have high doubling times, and lower specific growth rates), compared to the four strains grown in the absence of Tc.

\subsubsection{Nitrogen limited growth assay of MSMEG_2196-pMind antisense/sense}

Growth experiments were conducted in triplicate with M. smegmatis WT, Ms/pMind, Ms/p2196 S and Ms/p2196 AS grown in HdeB $\mathrm{N}^{-}$limited media containing $0 \mathrm{ng} / \mathrm{mL}$ and $40 \mathrm{ng} / \mathrm{mL}$ of Tc. The concentration of nitrogen in the HdeB media was reduced from $100 \mathrm{mM}$ to $0.01 \mathrm{mM}$, and the standard errors were produced as described in 3.3.1. $\mathrm{OD}_{600}$ absorbance readings were taken every 90 mins for the first $10 \mathrm{hrs}$ then every $3 \mathrm{hrs}$ daily for 2 days. The growth curves of all four cultures are shown in figure 3.3.5. The specific growth rates and doubling times were also calculated, and they are displayed in table 3.3.5. 


\section{Chapter 3: Results}

From the growth curves and the calculated doubling times, it is clear that $M$. smegmatis WT (in the absence and presence of Tc) grew at a much faster rate at both exponential- and stationary-phase (low doubling times), compared to Ms/pMind. This observation is supported by the final $\mathrm{OD}_{600}$ readings, where $M$. smegmatis WT reached a final $\mathrm{OD}_{600}$ (at 3,390 mins) of $1.18(\mathrm{Tc}+)$, and $1.09 \quad(\mathrm{Tc}-)$, compared to Ms/pMind final $\mathrm{OD}_{600}$ of $0.54(\mathrm{Tc}+)$, and $0.67(\mathrm{Tc}-)$. Ms/pMind and Ms/p2196 S culture, in both the presence and absence of Tc, had no significant effect (outside of standard errors there is no significant difference) on growth rate, which is clearly shown in the specific growth rates and doubling times calculated (Figure 3.3.5 and Table 3.3.5).

Ms/p2196 AS culture, grown in the presence of Tc, had the most marked decrease in $\mathrm{OD}_{600}$ readings under both $0 \mathrm{ng} / \mathrm{mL}$ and $40 \mathrm{ng} / \mathrm{mL}$ of Tc up until $\sim 1500$ mins $\left(\mathrm{OD}_{600}\right.$ of $0.62[\mathrm{Tc}-]$, and $\left.0.51[\mathrm{Tc}+]\right)$, compared to $\mathrm{Ms} / \mathrm{pMind}\left(\mathrm{OD}_{600}\right.$ of 0.97 [Tc-], and $1.12[\mathrm{Tc}+])$. After 2,000 mins, Ms/p2196 AS culture grown in the absence of Tc recovered their growth rate and seemed to have reached similar $\mathrm{OD}_{600}$ to that of Ms/pMind and Ms/p2196 S. However in the presence of $40 \mathrm{ng} / \mathrm{mL}$ of Tc, the $\mathrm{OD}_{600}$ of Ms/p2196 AS began to decrease just before 2,000 mins and kept decreasing steadily (540 mins of doubling time compared to 323 mins of doubling time for Ms/pMind at log-phase). Overall, the growth of Ms/p2196 AS culture was hindered under $\mathrm{N}^{-}$limitations in the presence of Tc, compared to the other three cultures. This growth curve was repeated twice (data not shown) and equivalent results were obtained. 


\section{Chapter 3: Results}

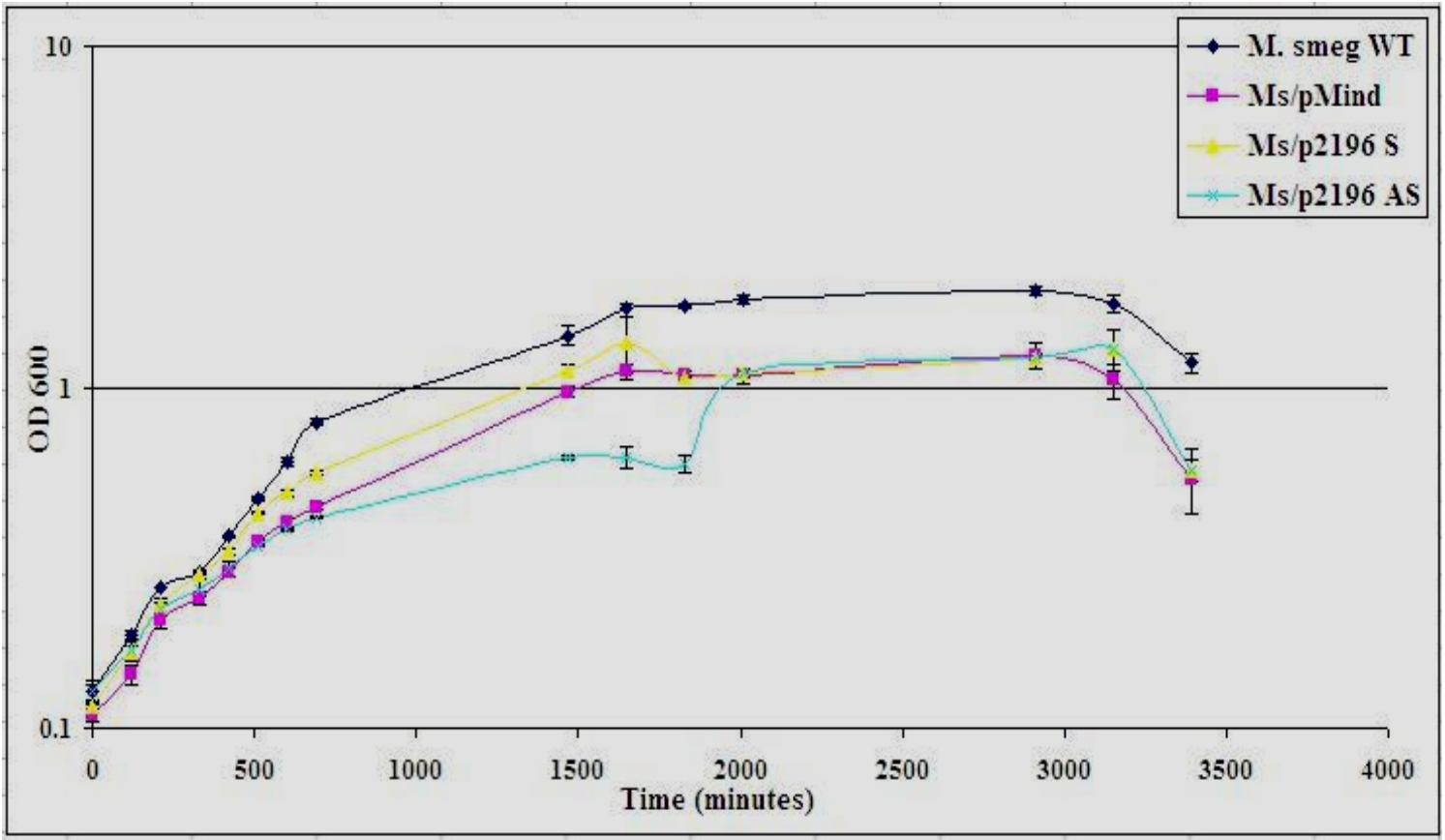

(B)

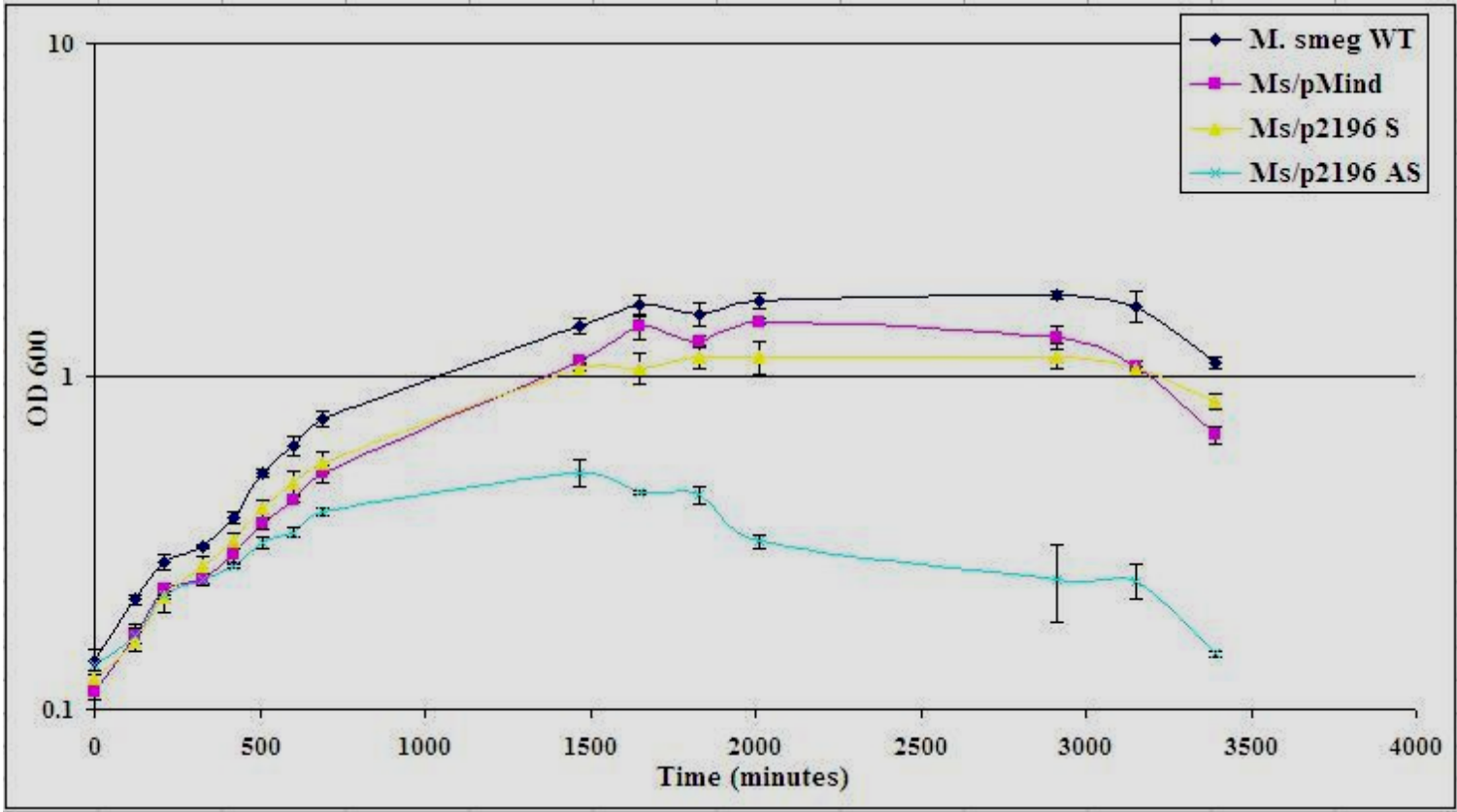

Figure 3.3.5: Growth curve of M. smegmatis WT, Ms/pMind, Ms/p2196 S, and Ms/p2196 AS grown in HdeB $\mathrm{N}^{-}$limited media. Growth of the four strains grown in $\mathrm{HdeB} \mathrm{N}^{-}$limited media containing either $0 \mathrm{ng} / \mathrm{mL}$ (A), or $40 \mathrm{ng} / \mathrm{mL}$ (B) of Tc. On the first day, the $\mathrm{OD}_{600}$ readings of the assays were taken every 90 mins, then every $3 \mathrm{hrs}$ for 2 days. The growth of Ms/p2196 AS culture grown in the presence of Tc (B) was affected significantly compared to Ms/p2196 AS in the absence of Tc (A). The results were plotted on a semi-log graph, and the standard errors were calculated by dividing the standard deviation by the square root of $\sqrt{3}$. 
$\underline{\text { Chapter 3: Results }}$

\begin{tabular}{|l|l|l|l|}
\hline $\begin{array}{l}\text { M. smegmatis } \\
\text { strain }\end{array}$ & Tc & $\begin{array}{l}\text { Log-phase specific growth } \\
\text { rate }\left(\mathbf{m i n}^{-1}\right)\end{array}$ & $\begin{array}{l}\text { Doubling time } \\
(\mathbf{m i n s})\end{array}$ \\
\hline M. smeg WT & - & $0.002767( \pm 0.0004680)$ & $250( \pm 42.30)$ \\
\hline M. smeg WT & + & $0.002739( \pm 0.0004047)$ & $253( \pm 37.37)$ \\
\hline Ms/pMind & - & $0.001851( \pm 0.0002728)$ & $374( \pm 55.08)$ \\
\hline Ms/pMind & + & $0.002145( \pm 0.0001486)$ & $323( \pm 22.38)$ \\
\hline Ms/p2196 S & - & $0.002196( \pm 0.0003802)$ & $316( \pm 54.67)$ \\
\hline Ms/p2196 S & + & $0.002253( \pm 0.0001309)$ & $308( \pm 17.90)$ \\
\hline Ms/p2196 AS & - & $0.001787( \pm 0.0001514)$ & $388( \pm 32.88)$ \\
\hline Ms/p2196 AS & + & $0.001281( \pm 0.0001833)$ & $540( \pm 77.25)$ \\
\hline
\end{tabular}

Table 3.3.5: Specific growth rates and doubling times for $\mathrm{N}^{-}$limited growth assay in HdeB

media. Growth rates and doubling times of the four cultures grown in the $\mathrm{HdeB} \mathrm{N}^{-}$limited media in the presence/absence of Tc. There is a significant difference (outside of the standard errors) in the doubling time of Ms/p2196 AS culture compared to the other three cultures. This demonstrates a growth effect in the Ms/p2196 AS grown under $\mathrm{N}^{-}$limitation due to the down-regulation of MSMEG_2196 in M. smegmatis.

\subsubsection{CFU of Ms/pMind and Ms/p2196 AS in $\mathrm{N}^{-}$limited culture}

$\mathrm{CFU}$, a measurement of viable bacterial cells, were preformed on Ms/pMind and Ms/p2196 AS cultures grown in HdeB $\mathrm{N}^{-}$limited media (both $0 \mathrm{ng} / \mathrm{mL}$ and 40ng/mL of Tc culture). $1 \mathrm{~mL}$ of cells at stationary-phase (1,440 mins and 2,880 mins) was collected for CFU analysis (see section 2.4.2), and the results of the CFU values are shown in table 3.3.6. At 1,440 mins, Ms/pMind CFUs ( $\mathrm{Tc}-$ and $\mathrm{Tc}+$ ) were greater than that of Ms/p2196 AS (Tc- and Tc+). At 2,880 mins, the CFU values of Ms/p2196 AS culture grown in the absence of Tc reached similar CFU values to that of Ms/pMind grown in both absence and presence of Tc. However, the CFUs of Ms/p2196 AS culture grown in the presence of $40 \mathrm{ng} / \mathrm{mL}$ Tc decreased further. The 
Chapter 3: Results

CFU values of both time points (1,440 and 2,880 mins) concur with the growth curve results shown in figure 3.3.5.

(A)

\begin{tabular}{|l|l|l|l|}
\hline $\begin{array}{l}\text { M. smegmatis } \\
\text { strain }\end{array}$ & Tc & $\begin{array}{l}\text { Cell concentration } \\
(\mathbf{C F U} / \mathbf{m L})\end{array}$ & Percentage \\
\hline $\mathrm{Ms} / \mathrm{pMind}$ & - & $1.823 \times 10^{8}( \pm 85.82)$ & $100.0 \%$ \\
\hline $\mathrm{Ms} / \mathrm{pMind}$ & + & $1.176 \times 10^{8}( \pm 8.09)$ & $64.5 \%$ \\
\hline $\mathrm{Ms} / \mathrm{p} 2196 \mathrm{AS}$ & - & $8.33 \times 10^{7}( \pm 8.65)$ & $45.7 \%$ \\
\hline $\mathrm{Ms} / \mathrm{p} 2196 \mathrm{AS}$ & + & $8.05 \times 10^{7}( \pm 1.50)$ & $44.2 \%$ \\
\hline
\end{tabular}

(B)

\begin{tabular}{|l|l|l|l|}
\hline $\begin{array}{l}\text { M. smegmatis } \\
\text { strain }\end{array}$ & Tc & $\begin{array}{l}\text { Cell concentration } \\
(\mathbf{C F U} / \mathbf{m L})\end{array}$ & Percentage \\
\hline $\mathrm{Ms} / \mathrm{pMind}$ & - & $2.48 \times 10^{8}( \pm 25.77)$ & $100.0 \%$ \\
\hline $\mathrm{Ms} / \mathrm{pMind}$ & + & $2.19 \times 10^{8}( \pm 11.00)$ & $88.3 \%$ \\
\hline $\mathrm{Ms} / \mathrm{p} 2196 \mathrm{AS}$ & - & $1.95 \times 10^{8}( \pm 19.73)$ & $78.6 \%$ \\
\hline $\mathrm{Ms} / \mathrm{p} 2196 \mathrm{AS}$ & + & $8.76 \times 10^{7}( \pm 1.45)$ & $35.3 \%$ \\
\hline
\end{tabular}

Table 3.3.6: CFU counts of Ms/pMind and Ms/p2196 AS grown in $\mathrm{N}^{-}$limited culture.

The CFU and percentage of Ms/pMind and Ms/p2196 AS culture grown in the $\mathrm{N}^{-}$limited media. The cultures were collected for CFU at 1,440 mins (A), and then again at 2,880 mins (B). 


\subsection{Proteomics of Ms/pMind and Ms/p2196 AS}

\subsubsection{Whole-cell protein extraction from Ms/pMind and Ms/p2196 AS culture for proteomic analysis}

The protein expression in Ms/p2196 AS culture grown under nitrogen limitation was analysed. Cells of all four cultures were harvested at stationary phase and stored at $-80{ }^{\circ} \mathrm{C}$. Since there were a significant differences (outside of standard errors) observed in Ms/p2196 AS Tc+ culture (in term of the $\mathrm{OD}_{600}$ absorbance readings and CFU values), these and $\mathrm{Ms} / \mathrm{pMind} \mathrm{Tc}+$ (used as control) cell culture were lysed using Triton X-100 and urea (see section 2.5.1). Protein quantitation was performed using the Pierce kit, and samples were run on SDS-PAGE gel to check for the quantity of the protein present in the whole cell lysates (Figure 3.4.1). The Triton-urea treated cell lysates of Ms/pMind and Ms/p2196 AS cultures grown under nitrogen-limited condition were selected for the 2D gel analysis. 


\section{Chapter 3: Results}

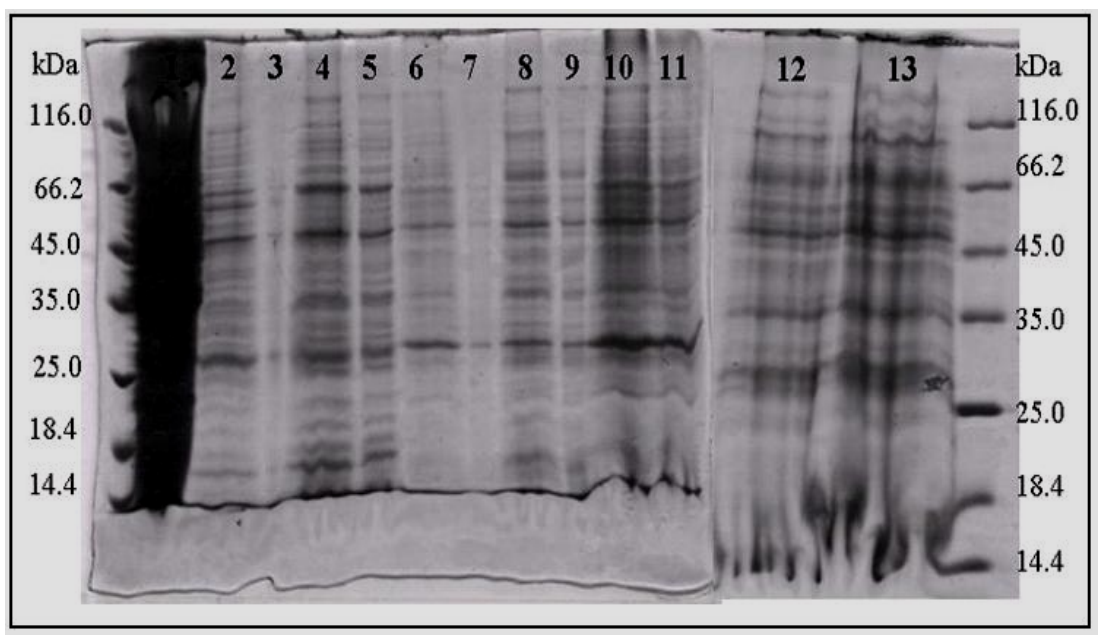

Figure 3.4.1: SDS-PAGE of whole cell lysates of Ms/pMind and Ms/p2196 AS cultures grown in nitrogen limited conditions. Ms/pMind and Ms/p2196 AS cell culture grown in $\mathrm{N}^{-}$limited media, in the presence and absences of Tc were harvested at stationary phase (at time 1,800 mins) and were prepared for cell lysis. Lane 1, control (MSMEG_2196/E.coli). Lanes 2 and 3, Ms/pMind Tc+ Triton X-100 treated (20 $\mu \mathrm{L}$ and $5 \mu \mathrm{L}$, respectively). Lanes 4 and 5, Ms/p2196 AS Tc+ Triton X-100 treated (20 $\mu \mathrm{L}$ and $5 \mu \mathrm{L}$, respectively). Lanes 6 and $7, \mathrm{Ms} / \mathrm{pMind} \mathrm{Tc}+$ Triton-urea treated $(20 \mu \mathrm{L}$ and $5 \mu \mathrm{L}$, respectively). Lanes 8 and 9, Ms/p2196 AS Tc+ Triton-urea treated (20 $\mu \mathrm{L}$ and $5 \mu \mathrm{L}$, respectively). Lanes 10 and 11, Ms/pMind Tc+ concentrated 5-fold ( $20 \mu \mathrm{L}$ and $5 \mu \mathrm{L}$, respectively). Lanes 12 and 13, Ms/p2196 AS Tc+ concentrated 5-fold (20 $\mu \mathrm{L}$ and $5 \mu \mathrm{L}$, respectively). The Triton/urea treated Ms/pMind and Ms/p2196 AS (in lanes 6 and 8) were used for 2-D gel electrophoresis.

\subsubsection{Analysis of differentially expressed proteins in Ms/pMind and Ms/p2196 AS strain grown in nitrogen-limited media}

The prepared protein samples of Ms/pMind (control) and Ms/p2196 AS culture grown in nitrogen limited media to stationary-phase were analysed by 2D gel electrophoresis. Two $p I$ ranges, 4-7 and 6-11, were used and analysed (see section 2.5.2 -2.5.4). The results obtained are shown in figure 3.4.2 and 3.4.3. 


\section{Chapter 3: Results}

The protein samples were analysed by 2D gel electrophoresis twice. 17 protein spots from pI 4-7 2D gels (7 from Ms/pMind and 10 from Ms/p2196 AS), and 31 protein spots from $p I$ 6-11 2D gels (7 from Ms/pMind and 24 from Ms/p2196 AS), were selected. The selection of the spots was determined using ImageMaster 2D Platinum 5.0 software (GE Healthcare/Amersham Biosciences). We were not specifically searching for particular proteins, hence the selection was based on protein spots being absent and/or present, up regulated and/or down regulated in either gel. To quantify the change in protein expression, the selected spot intensities were quantified using ImageJ software. In cases where the spot appeared and/or disappeared on either gel, baseline measurements were still detectable even though spots were not visible by eye. In these cases, the protein expression ratio calculated was high (between 20 and 30). Spots of interest were trypsin digested and the resulting peptides analysed by MALDI-ToF-MS. 


\section{Chapter 3: Results}

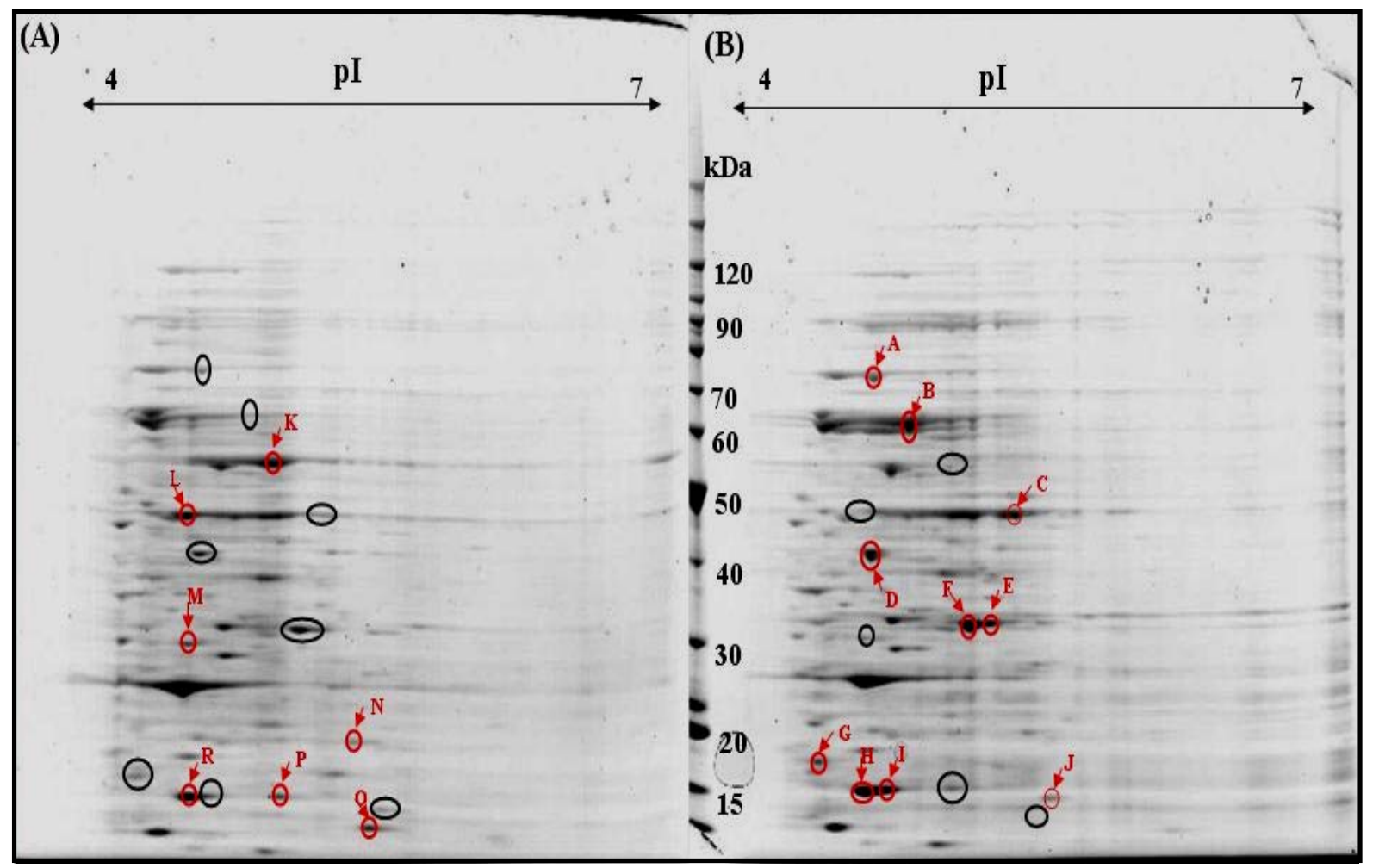

Figure 3.4.2: pH 4-7 2D gel electrophoresis of Ms/pMind (A) and Ms/p2196 AS (B).

Colloidal coomassie-stained 2D gel of total protein isolated from stationary phase of $M$. smegmatis containing Ms/pMind (A) used as control and Ms/p2196 AS (B) grown in nitrogen limited media. The protein spots on the two gels were compared using ImageMaster 2D platinum 5.0 software (GE Healthcare/Amersham Biosciences, New Zealand). The red-circled spots were selected by the software as being expressed in one gel but not the other. The black circles represent the proteins indicated by red circles in the opposite gel. There were some prominent spots that were present in both gels, such as spots A, D, E, F, G, and H; these were detected by ImageMaster software as being "not-identical", however when observing these spots by eye, they seem to be identical spots. 


\section{Chapter 3: Results}

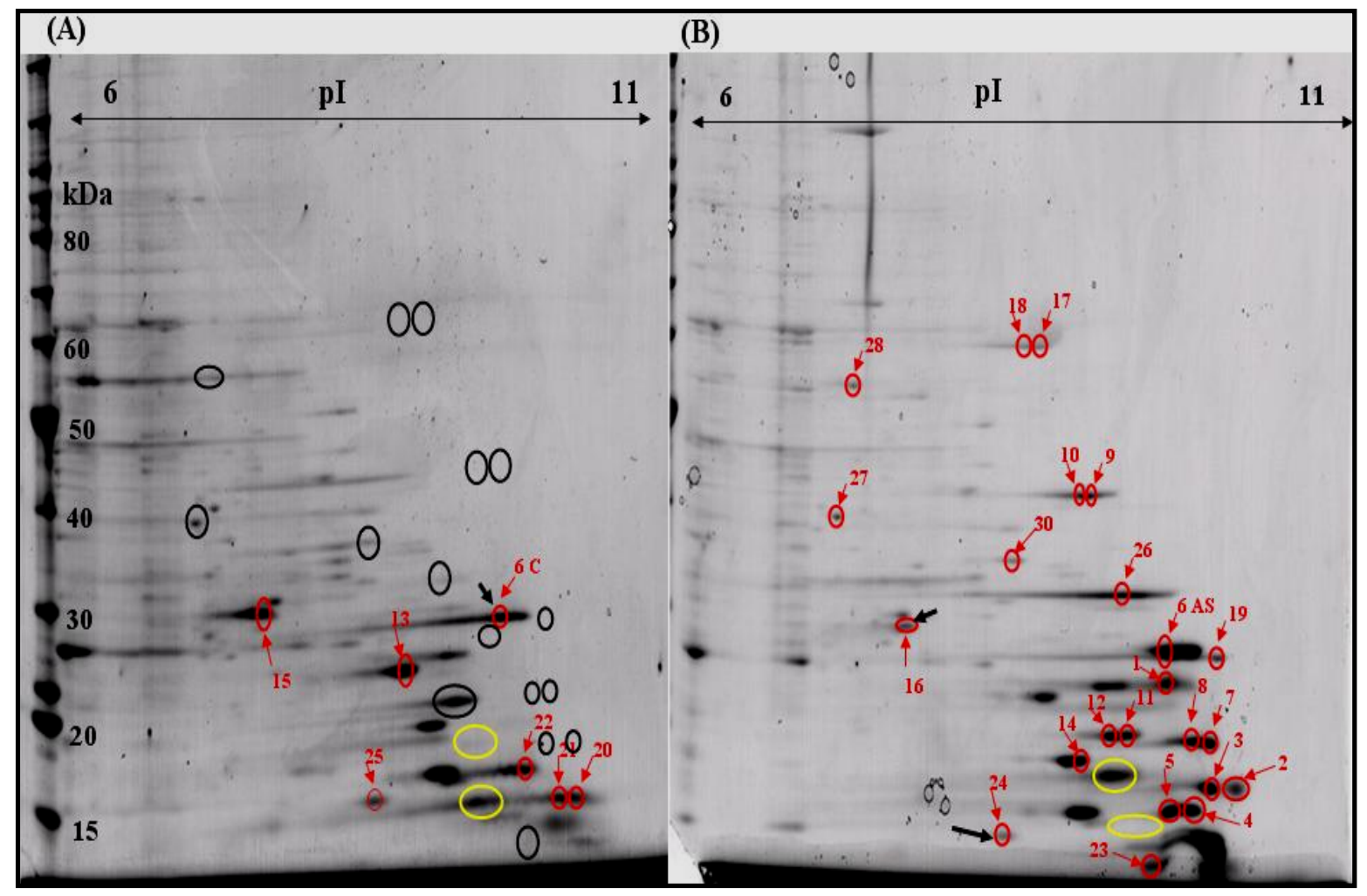

Figure 3.4.3: pH 6-11 2D gel electrophoresis of Ms/pMind (A) and Ms/p2196 AS (B).

Colloidal coomassie-stained 2D gel of total protein isolated from stationary phase of $M$. smegmatis containing Ms/pMind (A) used as control and Ms/p2196 AS (B) grown in nitrogen-limited media. The protein spots on the two gels were compared and selected using ImageMaster 2D platinum 5.0 software (GE Healthcare/Amersham Biosciences, New Zealand). The red-circled spots were identified by the software as being present in one gel but not the other. The black circles represent the red circles in the opposite gel. Black arrows represent spots selected by ImageMaster in both gels as being different however when identified, the proteins were identical. The yellow circles are spots not detected by the ImageMaster software however when comparing the two gels by eye, they are detected in one gel but not the other. These latter spots were not analysed. 


\section{Chapter 3: Results}

\subsubsection{Proteins identified on 2D gel}

For protein identifications of Ms/pMind and Ms/p2196 AS, the resulting mass spectra were analysed using the Mascot search engine database, from Science Matrix website (see section 2.5.5). The identified proteins are listed in table 3.4.1. Protein identification was significant (measure of the statistical significance of a match) if the protein scores were higher than 66 [88]. This is called the "score distribution" which is given by a histogram in the Mascot search result page. If a search is included in the green shaded area then there is greater than a $5 \%$ probability that the identification was random, conversely if search is outside the green shaded area than the matched protein had less than a $5 \%$ probability of being a random event [88]. The molecular weight, $p I$ values, and expectation values were other parameters looked at to check that proteins were not random matches. The expectation value is the "number of time we would expect to obtain an equal or higher score, purely by chance" [88], the lower the expectation value the more significant the result is. Most of the proteins identified in this study had a low expect value, which is encouraging. Furthermore, molecular weight and $p I$ values of each identified protein matches the spot being analyzed on the $2 \mathrm{D}$ gels, which add further confidence to the predicted protein match. Figure 3.4.4 gives an example of a Mascot search results. The database search identified $41 \%(\mathrm{pH} \mathrm{4-7} \mathrm{2D} \mathrm{gels)} \mathrm{and} 46 \%(\mathrm{pH} \mathrm{6-11} 2 \mathrm{D}$ gels) of proteins searched

for belonging to $M$. smegmatis $\mathrm{mc}^{2} 155$ strain. Not all of the proteins selected by MasterImage were identified. This could be due to the M. smegmatis genome not being completely annotated at the time when this study was conducted. 


\section{Probability Based Mowse Score}

Protein score is $-10^{*} \log (\mathrm{P})$, where $\mathrm{P}$ is the probability that the observed match is a random event. Protein scores greater than 66 are significant $(p<0.05)$.

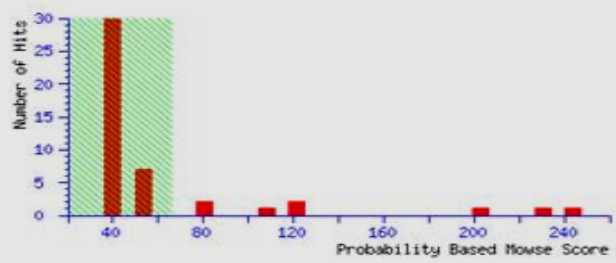

\section{Concise Protein Summary Report}

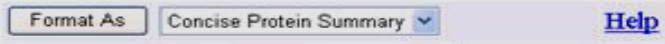

Significance threshold $\mathbf{p}<0.05 \quad$ Max. number of hits 10

Re-Search All Search Unmatched

1. Mixture 1 Total score: 244 Expect: $8.4 e-20$ queries matched: 41 components (only one family member shown for each component) :

qil1184728 3 Mass 53283 score 122 Expect: $1.3 e-07$ queries matched: 23

$30 \mathrm{~S}$ ribosomal protein $\mathrm{S} 1$ [Mycobacterium smegmatis str. MC2 155]

qi|118471714 Mass: 51624 Score: 113 Expect: $1.1 e-06$ Queries matched: 19

\section{\{SAIENCEX Mascot Search Results}

Protein View

Natch to: g11118472813 score: 122 Expect: $1.3 e-07$

$30 S$ ribosomal protein $\$ 1$ [Mycobacterium smegmatis str. MC2 155 ]

wominal mass $\left(\mathrm{K}_{\mathrm{n}}\right)$ : 53283; Calculated of value: 4.77

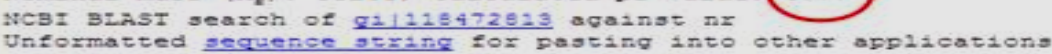

Taxolomy: Mycobsctexlum smeqmatis gex. MC2 155 , sequence from NCBI Enerez:

g11118174100 rrom Mycobacterium smegmatis str. MC2 iss

Fixed modilications: Carbamidomethyl (C)

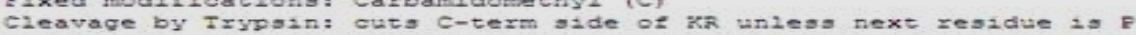

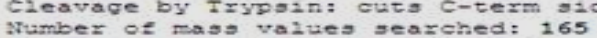

Number of pass ralued mafeped: 23

sequence coverace: 504

Marched peptides shown in Bold Recl

1 MPSPSUISPQ VAVUDIGSAE DELAMIDKTI KYFNDGDIVE GITVRVDRDE

51 VLLDIGYKTE GUIPSRELSI KMDVDPISYY SYGDEVBALV LTIEDKEGRI

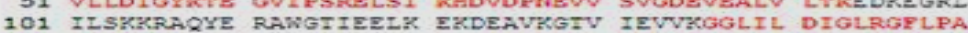

151 SLVEMRRVRD TOPYIGKEIE ARIIELDIOIR INTVYLSRRAW LEOTOSEVRS

201 BFLNQLQRGA IRKGVVSSIV NFGAFUDIGG VDGLVRVSEL SWRHIDHPSE

251 VVQVGDEVIV EVIDVDMDRE RVSISTRATQ EDPWRHEART HATGQTVPGE

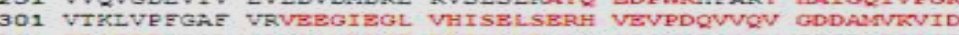

351 IDLERRRISE SIRQAIIEDYT EBFDRSTYGM ADSYDERGNY IFPEGFDPET

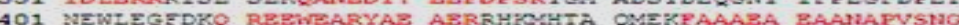

451 SSRSBZSSGG TLASDARLAA LREKLAOIA

Figure 3.4.4: Mascot search result of an identified protein. An example of Mascot search result obtained when identifying a protein. The top section of the result page is the probability based Mowse score. In this page the histogram probability of proteins identified (in the above example these are the red bar graph, outside the green shaded area) are significant. The protein summary report, below the histogram, gives further details about the identified protein such as the mass of the protein (red circle), score number (black circle), and the expect value (red circle). It also gives the name of the species for the identified protein, in this case M. smegmatis strain $\mathrm{mc}^{2} 155$ (red arrow). To obtain more information on the identified protein, clicking on the accession number (red rectangle) will open a new window called "protein view" (bottom). In this window, the pI value of the identified protein can be checked against the protein spot localisation on the $2 \mathrm{D}$ gel, and matched peptides are given in this page. 
Table 3.4.1: Proteins identified from 2D gel of Ms/pMind and Ms/p2196 AS

\begin{tabular}{|c|c|c|c|c|c|c|c|}
\hline Function $^{\mathrm{a}}$ & Identity/homology ${ }^{b}$ & $\begin{array}{l}\text { Spot labels } \\
\text { from Figs } 3.4 .2 \\
\text { and } 3.4 .3^{c}\end{array}$ & $\begin{array}{l}\text { Expression in } \\
\text { Ms/p2196 AS } \\
\text { (fold) }\end{array}$ & pI & $\begin{array}{l}\text { Molecular mass } \\
\text { (Da) }\end{array}$ & E-value ${ }^{e}$ & $\begin{array}{l}\text { Protein } \\
\text { score }\end{array}$ \\
\hline $\begin{array}{l}\text { RNA synthesis } \\
\text { and DNA synthesis }\end{array}$ & $\begin{array}{l}\text { DNA-directed RNA } \\
\text { polymerase } \alpha \text {-subunit. }\end{array}$ & D & $+(1.40)$ & 4.62 & 37954 & $4.4 e-05$ & 97 \\
\hline $\begin{array}{l}\text { Adaptations and } \\
\text { atypical condition }\end{array}$ & $\begin{array}{l}\text { Cold-shock binding } \\
\text { domain protein. }\end{array}$ & 24 & $-(1.50)$ & 6.86 & 16623 & 0.00024 & 90 \\
\hline \multirow[t]{2}{*}{$\begin{array}{l}\text { Repressors/ } \\
\text { activators }\end{array}$} & $\begin{array}{l}\text { Transcriptional regulator } \\
\text { Crp/Fnr family protein. }\end{array}$ & 13 & $-(1.20)$ & 9.57 & 24760 & $1.1 \mathrm{e}-11$ & 163 \\
\hline & $\begin{array}{l}\text { Nitrogen regulatory } \\
\text { protein P-II. }\end{array}$ & $\mathbf{O}$ & - (13.90) & 5.82 & 12108 & 0.36 & 65 \\
\hline \multirow{7}{*}{$\begin{array}{l}\text { Ribosomal protein } \\
\text { synthesis and } \\
\text { modification }\end{array}$} & Ribosomal protein $\mathbf{S 7}$ & 2 & $+(\mathbf{1 3 . 3 0})$ & 10.5 & 17618 & $1.3 e-09$ & 142 \\
\hline & 50S ribosomal protein L6 & 7 & $+(31.40)$ & 9.99 & 19442 & $4.2 \mathrm{e}-07$ & 117 \\
\hline & Ribosomal protein L14 & $20 / 21$ & $-(32.00)$ & 0.06 & 13425 & $1.3 \mathrm{e}-06$ & 112 \\
\hline & Ribosomal protein L10 & 14 & $+(1.15)$ & 9.00 & 18000 & $5.3 e-09$ & 136 \\
\hline & Ribosomal protein L1 & 6 & $+(1.70)$ & 9.60 & 25004 & $1.7 e-10$ & 151 \\
\hline & Ribosomal protein $\mathrm{S} 10$ & 23 & $+(5.80)$ & 9.41 & 11483 & $3.3 \mathrm{e}-05$ & 98 \\
\hline & Ribosomal protein L11 & 5 & $+(52.4)$ & 9.52 & 15050 & 0.00520 & 76 \\
\hline \multirow[t]{2}{*}{$\begin{array}{l}\text { Protein translation } \\
\text { and modification }\end{array}$} & $\begin{array}{l}\text { Translation elongation } \\
\text { factor } T u \text {. }\end{array}$ & $\mathbf{C}$ & $+(4.20)$ & 5.18 & 43709 & 0.00450 & 77 \\
\hline & $\begin{array}{l}\text { Peptidyl-prolyl cis-trans } \\
\text { isomerase } \mathrm{B} \text {. }\end{array}$ & $\mathbf{N}$ & $=$ & 5.42 & 18714 & $4.2 \mathrm{e}-07$ & 117 \\
\hline $\begin{array}{l}\text { Chaperons } \\
\text { heat shock }\end{array}$ & Chaperon protein DnaK & $\mathbf{A}$ & $+(\mathbf{1 . 8 0})$ & 4.71 & 66606 & 2.1e-15 & 200 \\
\hline $\begin{array}{l}\text { Biosynthesis of } \\
\text { Cofactor }\end{array}$ & Geranylgeranyl reductase & 10 & $+(28.00)$ & 9.30 & 43182 & 0.00033 & 88 \\
\hline $\begin{array}{l}\text { Ribosome } \\
\text { modification } \\
\text { and maturation }\end{array}$ & $\begin{array}{l}\text { Dimethyladenosine } \\
\text { transferase }\end{array}$ & 30 & $+(1.40)$ & 7.12 & 32813 & 0.013 & 72 \\
\hline \multirow[t]{2}{*}{$\begin{array}{l}\text { Amino acid } \\
\text { biosynthesis }\end{array}$} & $\begin{array}{l}\text { Glutamine synthetase } \\
\text { type I. }\end{array}$ & $\mathbf{K}$ & - (11.30) & 4.97 & 53729 & $4.2 \mathrm{e}-06$ & 107 \\
\hline & Glutamate 5-kinase. & 27 & $-(11.30)$ & 6.77 & 38583 & 0.0046 & 77 \\
\hline Energy metabolism & $\begin{array}{l}\text { Malate: quinine- } \\
\text { oxidoreductase }\end{array}$ & 28 & $=$ & 7.11 & 54993 & $1.2 \mathrm{e}-06$ & 52 \\
\hline Others & TROVE domain protein & 18 & $+(4.40)$ & 9.29 & 61373 & $1.1 \mathrm{e}-13$ & 183 \\
\hline
\end{tabular}




\section{Chapter 3: Results}

Table 3.4.1: Proteins identified from 2D gel of Ms/pMind and Ms/p2196 AS.

All of the protein spots from the 2D gel (Fig 3.4.2 and 3.4.3) that were identifiable using the Mascot search engine (Science matrix database). An explanatory footnote is given below for the headings presented inside table 3.4.1.

${ }^{\text {a }}$ Functional categories given by the Mascot search database from Science matrix.

${ }^{b}$ Functions as described in "The Institute of Genomic Research (TIGR) database for fully identified gene products or proposed function and/or homology for the others.

${ }^{\mathrm{c}}$ Spot labels: letters according to those in Fig. 3.4.2; numbers according to those in Fig. 3.4.3.

${ }^{\mathrm{d}}$ Level of expression for each spot determined using ImageJ.

“+”, up-regulated proteins; "-“, down-regulated proteins; "=", proteins whom expression level was unchanged (in this case a protein spot having a ratio of 1 or less was labelled as having an equal amount of expression level in either the Ms/pMind or Ms/p2196 AS). The ratio rates are indicated in brackets.

${ }^{\mathrm{e}} \mathrm{E}$-value is the number of times we would expect to obtain an equal or higher score, purely by chance. The lower the E-value is the significant the results are. 


\subsection{Site directed mutagenesis of Mb1389c and MSMEG_2196}

\subsubsection{PCR of Mb1389c and MSMEG_2196 flanking regions}

To delete the Mb1389c (M. bovis) and MSMEG_2196 (M. smegmatis) genes, overlap PCR was used, where the flanking regions of the gene in question are amplified first. Since identical work was carried out on both M. bovis and M. smegmatis, in term of constructing the mutants, both were included in the same sections (see Materials and Methods sections 2.6.1-2.6.10). The flanking regions ( $600 \mathrm{bp})$ were amplified using the primers listed in table 2.6.1, and PCR products were run on $1 \%$ agarose gel (Figure 3.5.1 and 3.5.2) and gel purified.

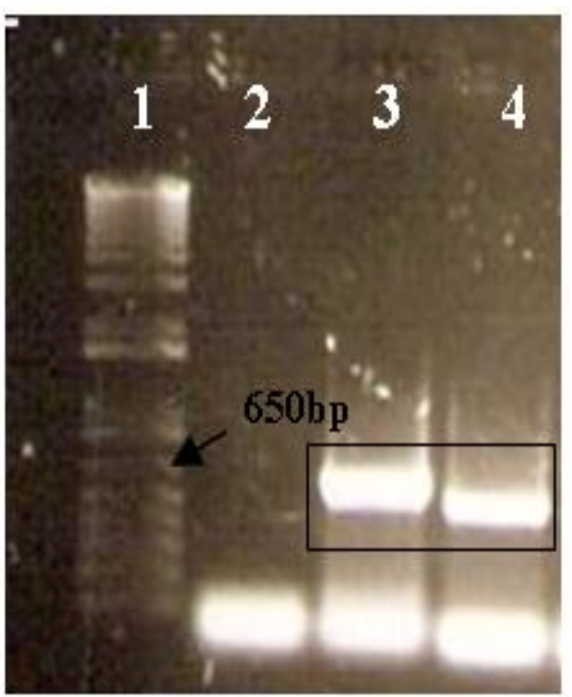

Figure 3.5.1: $1 \%$ agarose gel of Mb1389c flanking region PCR. Mb1389c upstream flanking region was amplified using Mb1389c C1/R4 primers (lane 3, 650 bp), and downstream flanking region was amplified using Mb1389c F2/D2 primers (lane 4, 650 bp). Lane 2 contains negative control; lane 1 contains $1 \mathrm{~Kb}+$ marker (Invitrogen, New Zealand). 


\section{Chapter 3: Results}

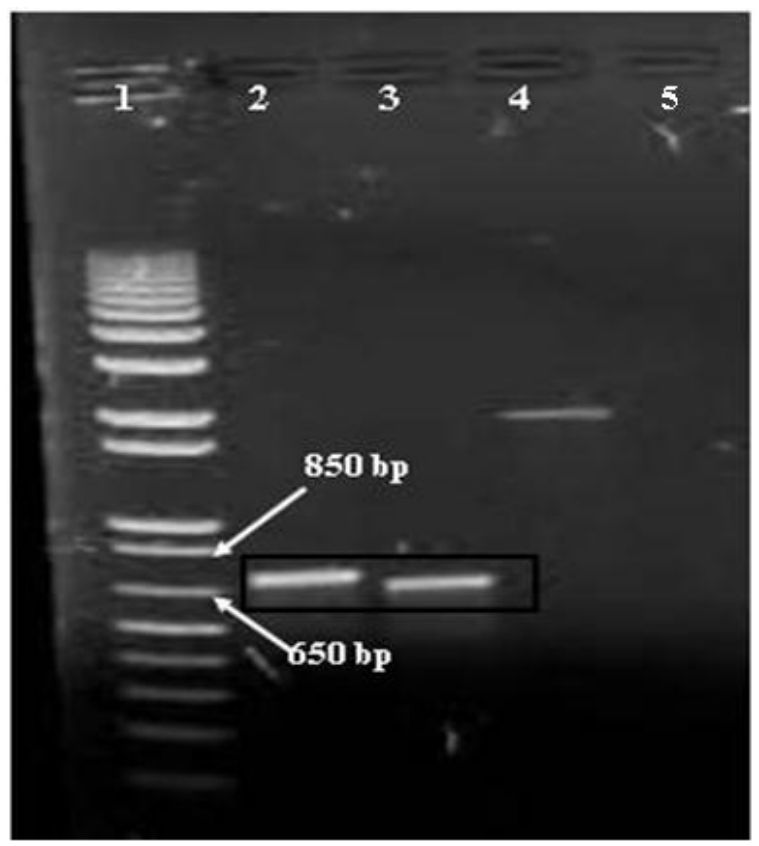

Figure 3.5.2: $1 \%$ agarose gel of MSMEG_2196 flanking region PCR. MSMEG_2196 upstream flanking region was amplified using Ms_2196 C11/R11 primers, lane $2(\sim 650 \mathrm{bp}, 10 \mu \mathrm{L} / 25 \mu \mathrm{L})$, and the downstream flanking region was amplified using Ms_2196 F13/R16 primers, lane $3(\sim 650 \mathrm{bp}, 10 \mu \mathrm{L} / 25 \mu \mathrm{L})$. Lane 4 contains positive control, and lane 5 contains negative control. Lane 1 contains $1 \mathrm{~Kb}+$ marker (Invitrogen, New Zealand).

\subsubsection{Overlap PCR of Mb1389c and MSMEG_2196}

Overlap PCR was used to join the upstream-amplified flanking region of $\mathrm{Mb} 1389 \mathrm{c} \mathrm{C} 1 / \mathrm{F} 4$ to the downstream flanking region of Mb1389c F2/D2 using methods previously described [56]. Initially, this protocol was also applied to join the upstream flanking region of Ms_2196 C11/R11 to the downstream flanking region Ms_2196 F13/R16 with no success hence a second protocol was used [23] (see section 2.6.3). This protocol was successful, and it gave the desired 1,200 bp product. The PCR products were run on $1 \%$ agarose gel and gel purified (Figure 3.5.3 and 3.5.4). 


\section{Chapter 3: Results}

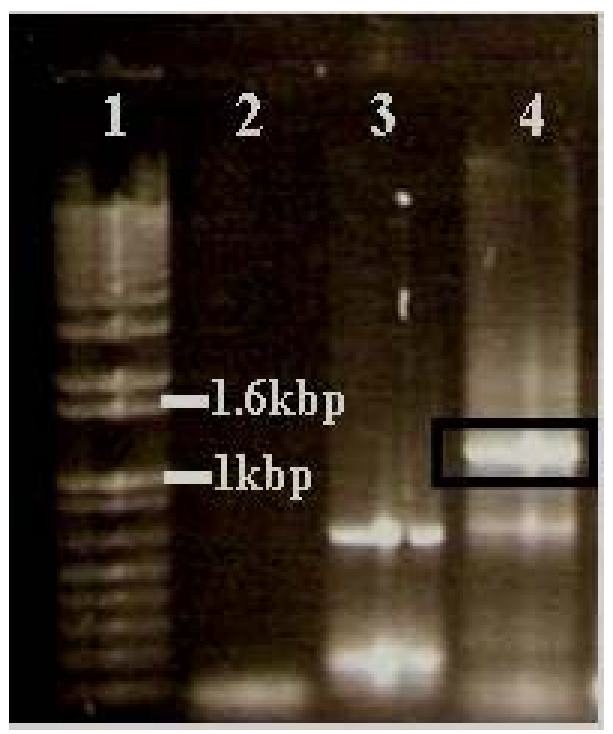

Figure 3.5.3: $1 \%$ agarose gel of Mb1389c overlap PCR. Mb1389c overlap PCR product was amplified using Mb1389c C1/D2 primers, lane 4 ( $1.2 \mathrm{kbp})$. Lane 2 contains negative control; lane 3 contains positive control. Lane 1 contains $1 \mathrm{~Kb}+$ marker (Invitrogen, New Zealand).

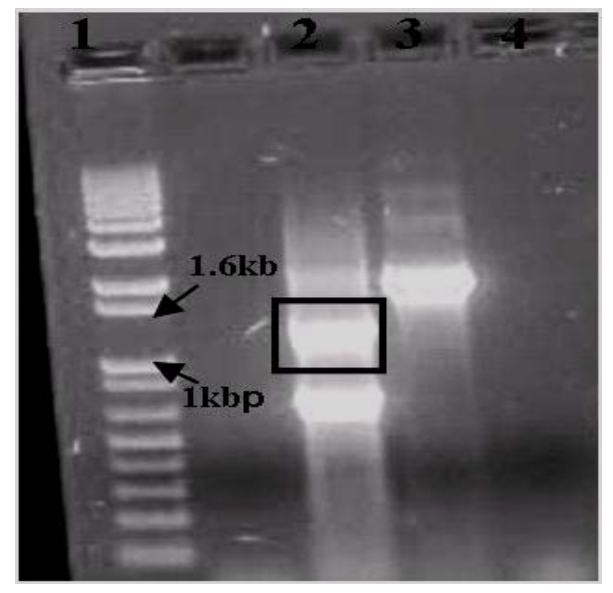

Figure 3.5.4: $1 \%$ agarose gel of MSMEG_2196 overlap PCR. Overlap fragment of MSMEG_2196 was amplified using Ms_2196 F11/R16 primers, lane $2(\sim 1.2 \mathrm{kbp}, 10 \mu \mathrm{L} / 30 \mu \mathrm{L})$. Lane 3 contains positive control, and lane 4 contains negative control. Lane 1 contains $1 \mathrm{~Kb}+$ marker (Invitrogen, New Zealand). 


\subsubsection{Cloning of Mb1389c- and MSMEG_2196-overlap fragment into pGEM-T}

\section{Easy cloning vector}

pGEM-T Easy cloning vector was used, into which the Mb1389c and MSMEG_2196 overlap PCR fragments were ligated. The resulting p1389c-overlap recombinant plasmids were digested with EcoR I to check for the correct Mb1389c insert $(\sim 1.2 \mathrm{kbp})$. The resulting p2196-overlap recombinant plasmids were digested with Pac I enzyme to check for the correct MSMEG_2196 insert ( 1.2kbp). The digested reaction was run on a $1 \%$ agarose gel (Figure 3.5.5 and 3.5.6).

The plasmids that contained Mb1389c and MSMEG_2196 1.2kbp fragment were sequenced by Allan Wilson Centre (New Zealand). The chromatograms of the sequences were corrected and examined for fidelity and absence of mutations (data not shown).

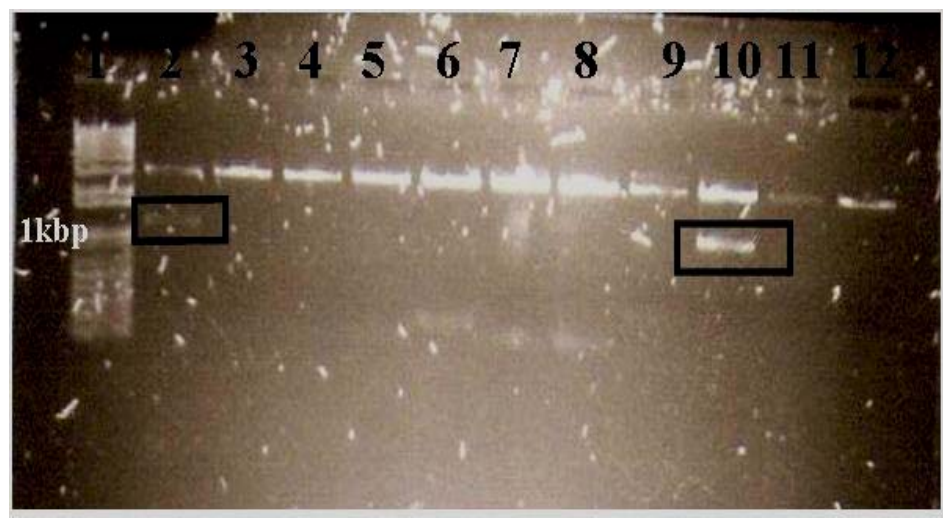

Figure 3.5.5: $1 \%$ agarose gel of p1389c-overlap EcoR I digested fragment. Lanes 2-12 contains p1389coverlap digested with EcoR I enzyme $(15 \mu \mathrm{L} / 25 \mu \mathrm{L})$. Inserts of Mb1389c are present in lanes 2 and 10 . Lane 1 contains $1 \mathrm{~Kb}+$ marker (Invitrogen, New Zealand). 


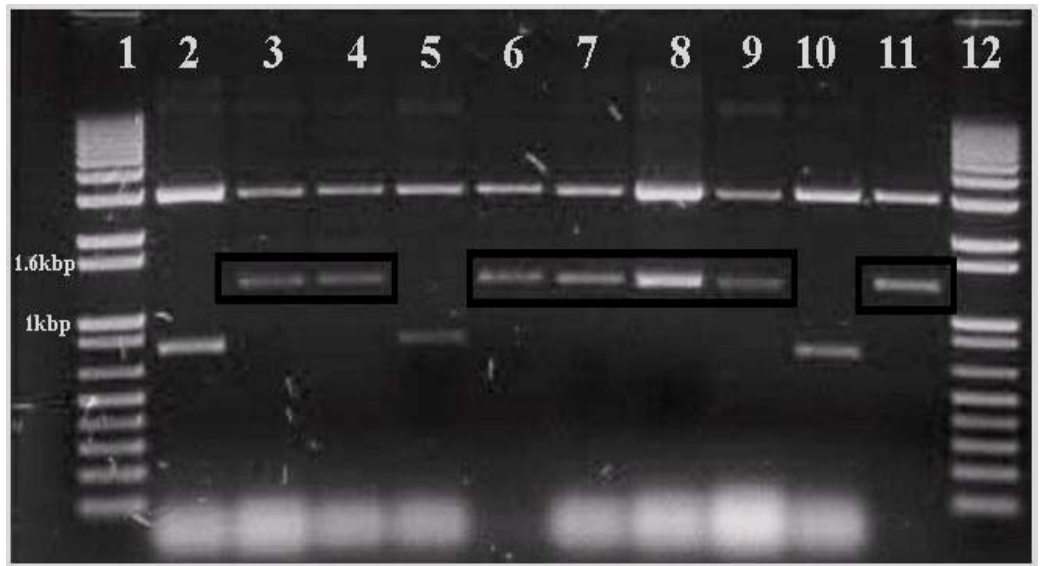

Figure 3.5.6: $1 \%$ agarose gel of p2196-overlap Pac I digested fragment. p2196-overlap plasmids were digested with Pac I enzyme, lane 2-11. MSMEG_2196 inserts present in lanes 3, 4, 6, 7, 8, 9, and 11. $20 \mu \mathrm{L} / 25 \mu \mathrm{L}$ per lane of Pac I digested reaction was run on gel. Lanes 1 and 12 contains $1 \mathrm{~Kb}+$ markers (Invitrogen, New Zealand).

\subsubsection{Digestion of Mb1389c- and MSMEG_2196-overlap fragment with Pac I enzyme for ligation into pUHA778 vector}

To prepare the Mb1389c- and MSMEG_2196-overlap PCR fragments for ligation into the knock-out vector pUHA778, the purified overlap PCR products were first digested with Pac I enzyme. The digested products were run on a $1 \%$ agarose gel, and they were gel purified (Figure 3.5.7, and 3.5.8). 


\section{Chapter 3: Results}

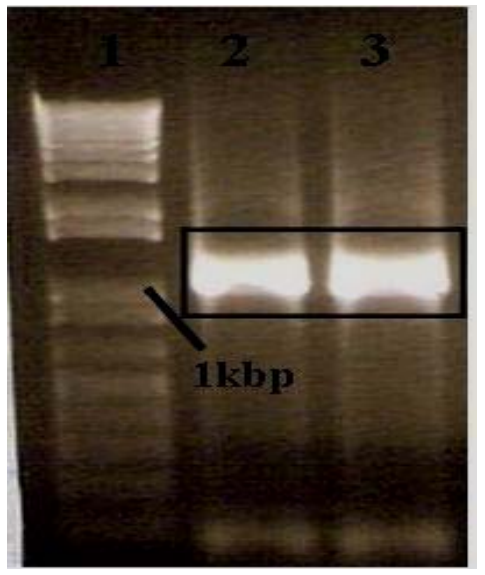

Figure 3.5.7: $1 \%$ agarose gel of Mb1389c-overlap Pac I digested fragment. The expected $\sim 1.2 \mathrm{kbp}$ digested product of Mb1389c-overlap (lanes 2 and 3). These products were gel purified.

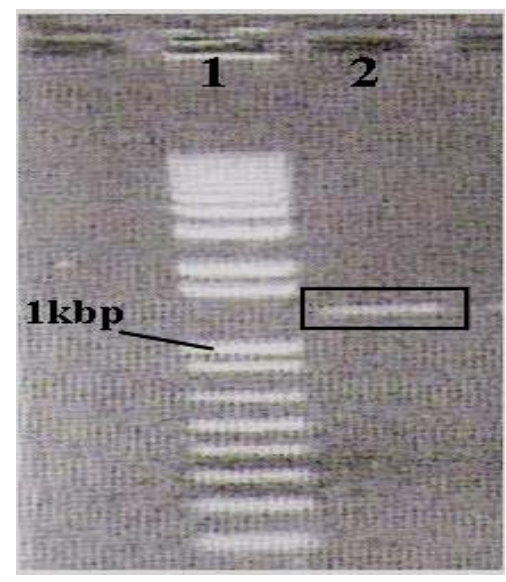

Figure 3.5.8: $1 \%$ agarose gel of MSMEG_2196-overlap Pac I digested fragment. The expected digested product of MSMEG_2196-overlap is $\sim 1.2 \mathrm{kbp}$ in lane 2. Lane 1 contains $1 \mathrm{~Kb}+$ marker (Invitrogen, New Zealand) 


\subsubsection{Ligation of Mb1389c- and MSMEG_2196-pverlap Pac I digested fragments into pUHA778 plasmid}

The ligation of Mb1389c- and MSMEG_2196-overlap fragments into pUHA778 plasmid generated pUHA778-Mb1389c and pUHA778-MSMEG_2196 fragments. These fragments were transformed into E. cloi DH5a cells (Section 2.6.6), and the resulting recombinant plasmids were digested with Pac I enzyme and run on $1 \%$ agarose gel (Figure 3.5.9 and 3.5.10), to check for the presence of insert.

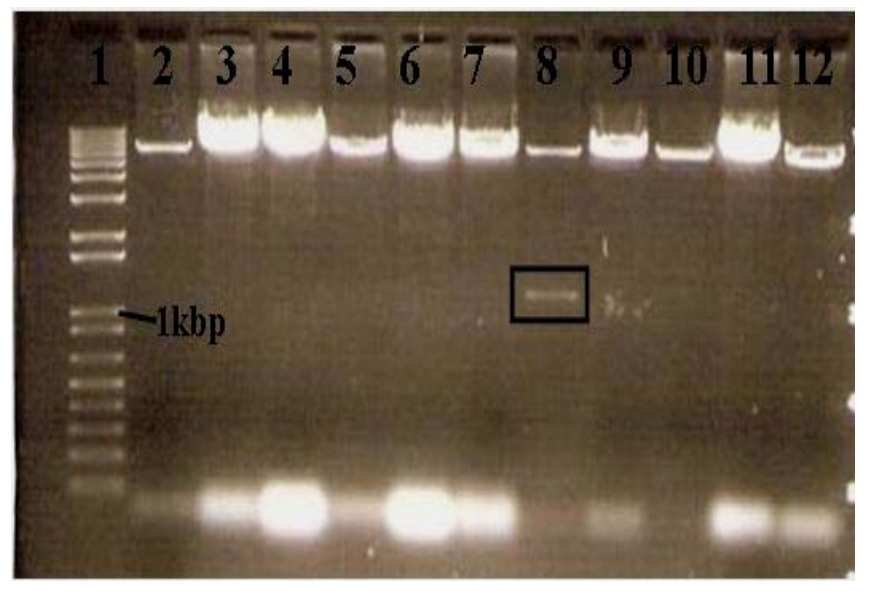

Figure 3.5.9: $1 \%$ agarose gel of Mb1389c-pUHA778 recombinant plasmids Pac I digested. Lane 1 contians $1 \mathrm{~Kb}+$ marker (Invitrogen, Auckland, New Zealand). Lanes 2-12 contains Mb1389c-pUHA778 plasmids digested with Pac I enzyme. Lane 8 contains Mb1389c insert. 


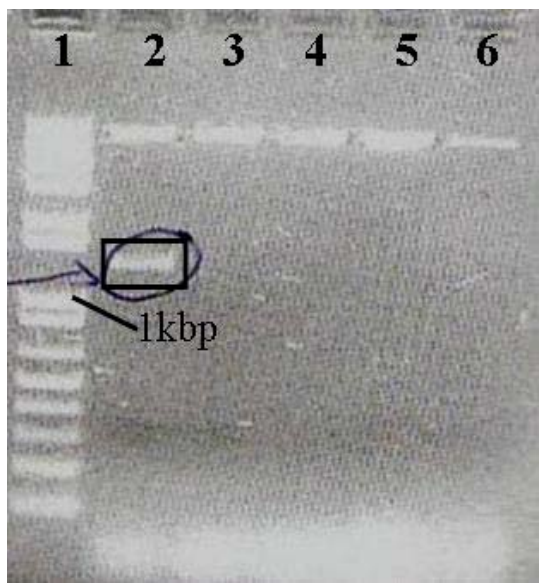

Figure 3.5.10: 1 \% agarose gel of MSMEG_2196-pUHA778 recombinant plasmids Pac I digested. Lanes 2-6 contains MSMEG_2196-pUHA778 plasmids. Lane 2 contains the desired MSMEG_2196 insert.

\subsubsection{Generation of $M$. bovis-1389c mutants}

Generation of M. bovis Mb1389c mutants was carried out at AgResearch Ltd. Wallaceville, New Zealand PC3 laboratory. At AgResearch PC3 laboratories, the Mb1389c-pUHA778 fragment was transformed in M. bovis (Section 2.6.8). Since $M$. bovis is a slow growing bacterium the transformants' incubation period took 3 weeks. The first resulting colonies of $M$. bovis $\Delta 1389$ were heat killed from which gDNA extraction was carried out and run on $1 \%$ agarose gel (Figure 3.5.11). To check for first cross-over and/or second cross-over PCR was carried out using primers shown in table 2.6.4, different combinations of these primers was used. Varying PCR conditions were applied such as gradient annealing temperature PCR, as well as normal PCR (Table 2.3.1); however, unspecific amplifications resulted each time (data not shown). Southern blot was carried out to screen for the first cross-over and/or knockouts of $\Delta 1389$ in $M$. bovis. gDNA of the first heat killed colonies were digested with $\mathrm{Bgl}$ II enzyme and run on $0.8 \%$ agarose gel (Figure 3.5.12) and then run on Southern blot (results not shown). 


\section{Chapter 3: Results}

The fragment size of Bgl II in wild type M. bovis1389c is $13,818 \mathrm{bp}$. Hence in first cross-over we are looking for a shift from the wild type 13,818 bp band to a larger band size that contains wild type fragment and the inserted DNA (this includes the 1,200 bp flanking regions plus 7,506 bp of pUHA778 plasmid) giving a total size of 22,524 bp figure 3.5.13. In the second cross over pUHA778 plasmid should not be present, giving either Mb1389c wild type gene (the reversion of Mb1389c gene to wild type) or the mutated Mb1389c gene, figure 3.5.14.

More heat killed colonies of $M$. bovis $\Delta 1389$ were treated as mentioned earlier for

the Southern blot. At this stage no first cross over and/or second cross over (knockout) of M. bovis $\Delta 1389$ were present in the samples obtained, it is possible that more colonies need to be screened for. Table 3.5.1 summarises the construction of the Mb1389c mutation in M. bovis. 


\section{Chapter 3: Results}

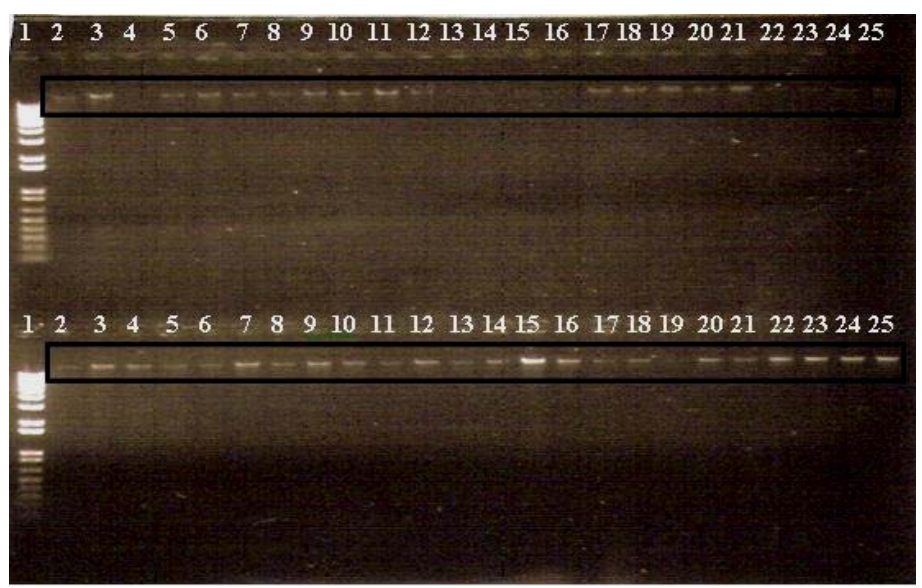

Figure 3.5.11: $1 \%$ agarose gel of gDNA of $M$. bovis $\mathbf{\Delta 1 3 8 9}$ colonies. Lanes 2-25 (top and bottom wells) contain gDNA of heat-killed M. bovis $\Delta 1389$ plasmids obtained from AgResearch. Lane 1 of the top and bottom halves of the gel contains $1 \mathrm{~kb}+$ marker (Invitrogen, New Zealand).

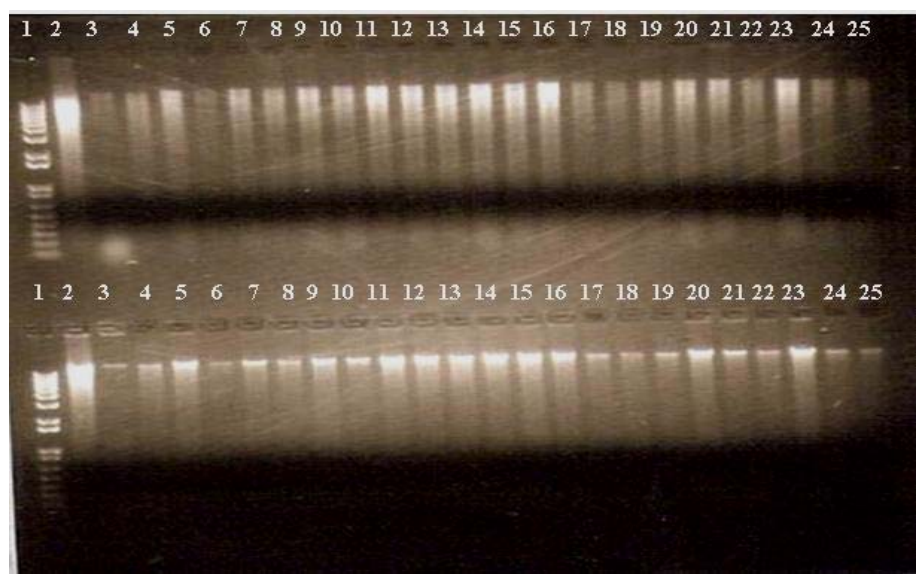

Figure 3.5.12: $0.8 \%$ agarose gel of $M$. bovis $\Delta 1389$ gDNA digested with Bgl II enzyme. Bgl II enzyme was used to digest $M$. bovis $\Delta 1389 \mathrm{gDNA}$ for southern blot analysis. Top wells, lanes 4-25 contain $\mathrm{Bgl}$ II digested samples, bottom wells contain the same samples however are not Bgl II digested. Lane 2 (top and bottom wells) contains wild type M. bovis gDNA, used as positive control. Lane 3 (top and bottom wells) contains wild type $M$. smegmatis gDNA, used as negative control. 


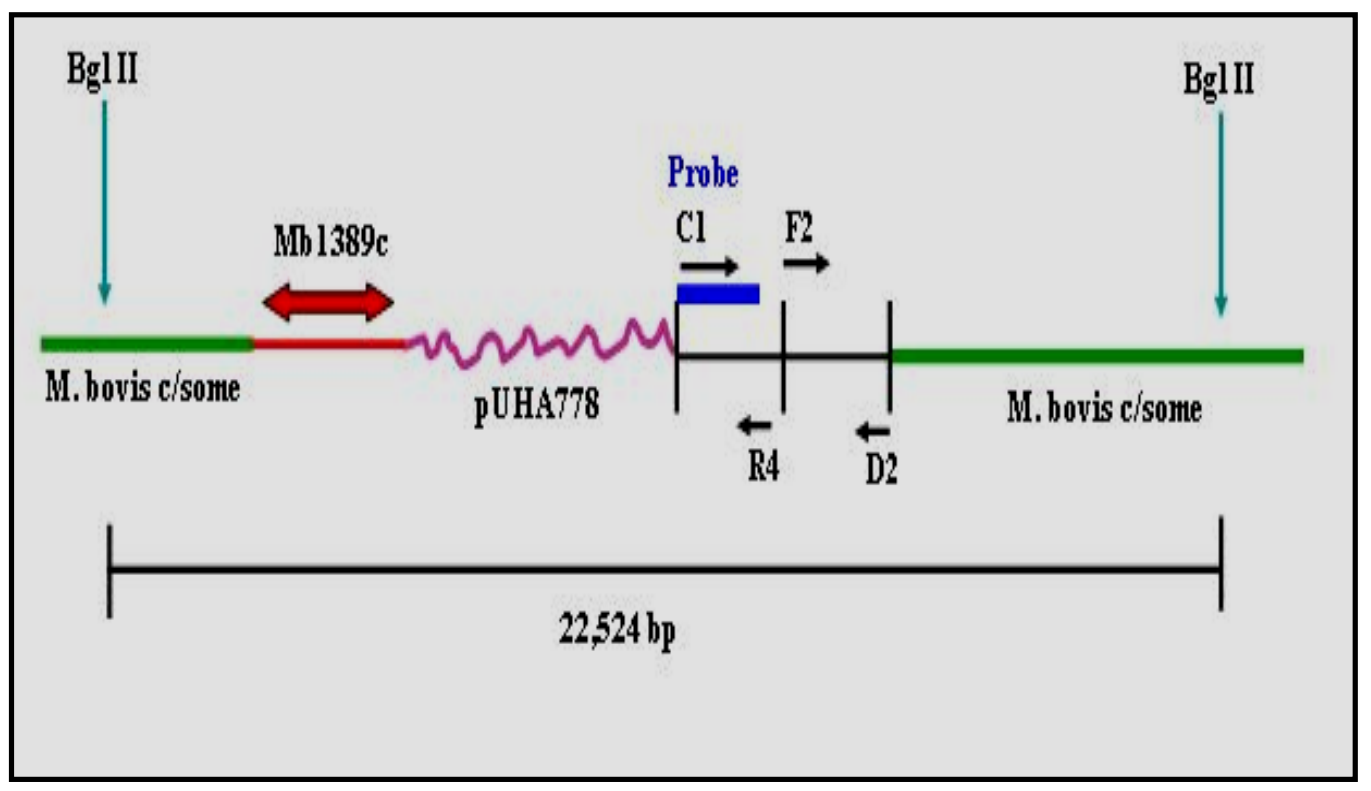

Figure 3.5.13: The expected fragment size of first crossover of Mb1389c-pUHA778 in Southern blot. The expected sizes would be wild type M. bovis 1389c ( 13,818 bp) plus pUHA778 plasmid ( 7,506 bp) and the flanking region fragment $(1,200 \mathrm{bp})$, giving a total fragment size of 22,524 bp. Lines not drawn to scale.
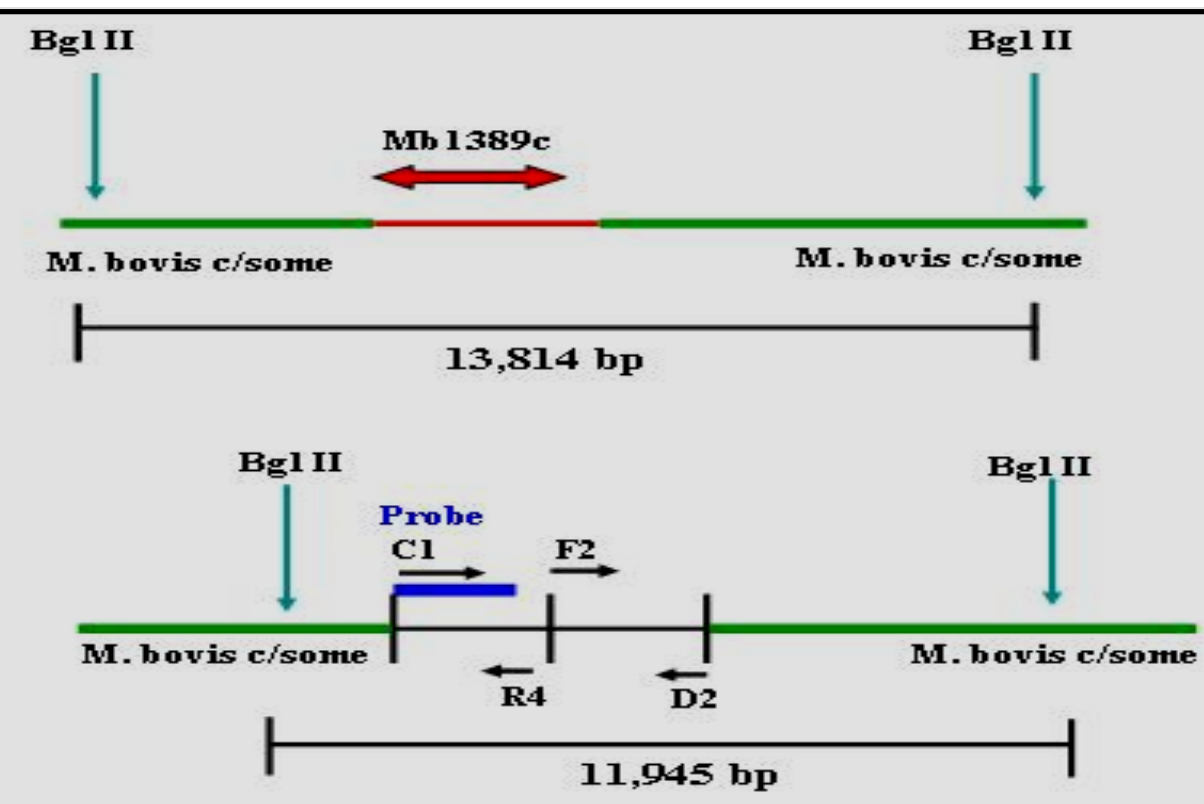

Figure 3.5.14: The expected fragment size of second crossover of Mb1389c-pUHA778 in Southern

blot. The possible fragments will be either the reversion of Mb1389c to wild type which will produce a fragment size of 13,184 bp (A). Alternatively, it will give the size of the mutated gene of Mb1389c, which will be 11,942 bp. Lines not drawn to scale. 


\subsubsection{Generation of $M$. smegmatis-2196 mutants}

The MSMEG_2196-pUHA778 plasmids, with the right insert, were transformed into M. smegmatis and plated out onto $\mathrm{LA} \mathrm{Hyg}_{50}$ agar plates. The transformation was carried out using a Bio-Rad E. coli pulser, where $\mathrm{kV}$ was the only variable, and the electroporator did not contain micro Faraday $(\mathrm{mF})$ and $\mathrm{Ohms}(\Omega)$ variable functions. The resulting colonies were inoculated into $3 \mathrm{~mL}$ of $\mathrm{LB}$ broth containing $\mathrm{Hyg}_{50}$ and $0.1 \%$ Tween 80 , to make stock culture and to extract gDNA.

Initially PCR was carried out to check for the presence of single homologues recombination and/or knockout of MSMEG_2196 in M. smegmatis. Primers used are shown in table 2.6.5, and combination of these primers was employed for PCR. The PCR amplification gave either a product similar to that of MSMEG_2196 wild type and/or unspecific amplifications, even though the positive control gave the correct fragment size (data not shown). Southern blot was applied; gDNA samples of M. smegmatis $\Delta 2196$ were digested with EcoN I enzyme and run on $0.8 \%$ agarose gel (Figure 3.5.15).

EcoN I enzyme cuts twice outside the MSMEG_2196 gene and it does not have digestion sites inside the pUHA778 plasmid. Hence, fragments of the first crossover and/or second cross-over should be readily obtained. EcoN I gives a fragment size of $5,398 \mathrm{bp}$ in the wild type MSMEG_2196; in the first crossover, we are expecting a shift from the wild type to a larger fragment of 14,054 bp (Figure 3.5.16). In the second crossover (gene knock out), the pUHA778 plasmid will be lost giving a fragment of either 5,398 bp (reversion to wild type) or a smaller fragment consisting of 3,303 bp (Figure 3.5.17). The EcoN I digested MSMEG_2196-pUHA778 gDNA were run on Southern blot (data not shown). The knockout fragment of MSMEG_2196 was not present in the Southern blot analysis; neither was in PCR reaction. More colonies need to 


\section{Chapter 3: Results}

be screened on Southern blot. Table 3.5.1 summarises the construction of the MSMEG_2196 mutant in M. smegmatis.

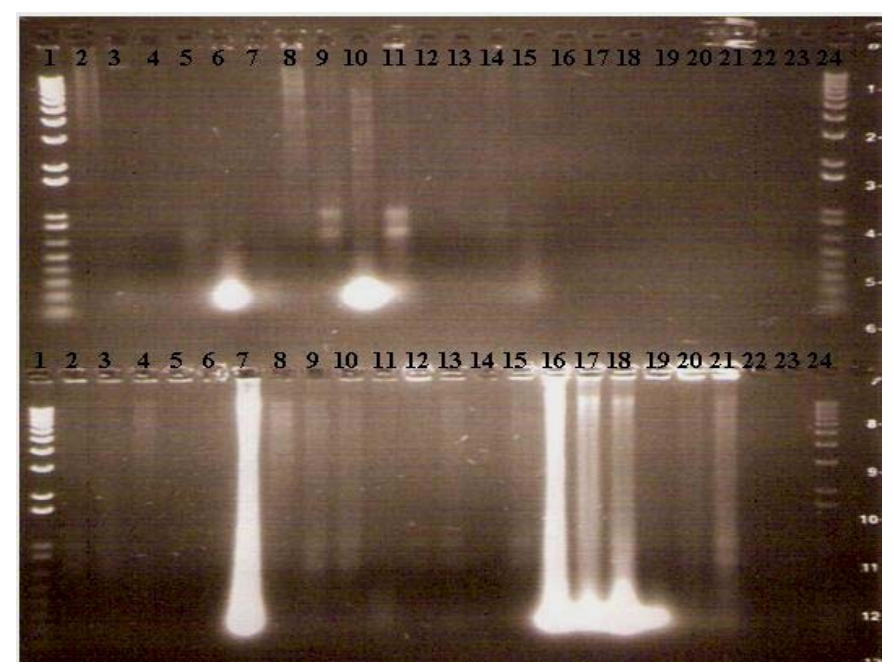

Figure 3.5.15: $0.8 \%$ agarose gel of gDNA of $M$. smegmatis $\Delta 2196$ digested with $E c o N$ I enzyme. The few resulting MSMEG_2196-pUHA778 colonies from the transformation were selected and gDNA was extracted. gDNA was digested by EcoN I. Lanes 5-23 (top wells) and lanes 2-23 (bottom wells) contain MSMEG_2196-pUHA778 EcoN I digests. Lanes 2, 3, and 4 (top wells) contains undigested M. bovis wild type; undigested M. smegmatis wild type, and Econ I digested M. smegmatis wild type gDNA, respectively. Lanes 1 and 24 (top and bottom wells) contains 1kb+ marker (Invitrogen, New Zealand). 


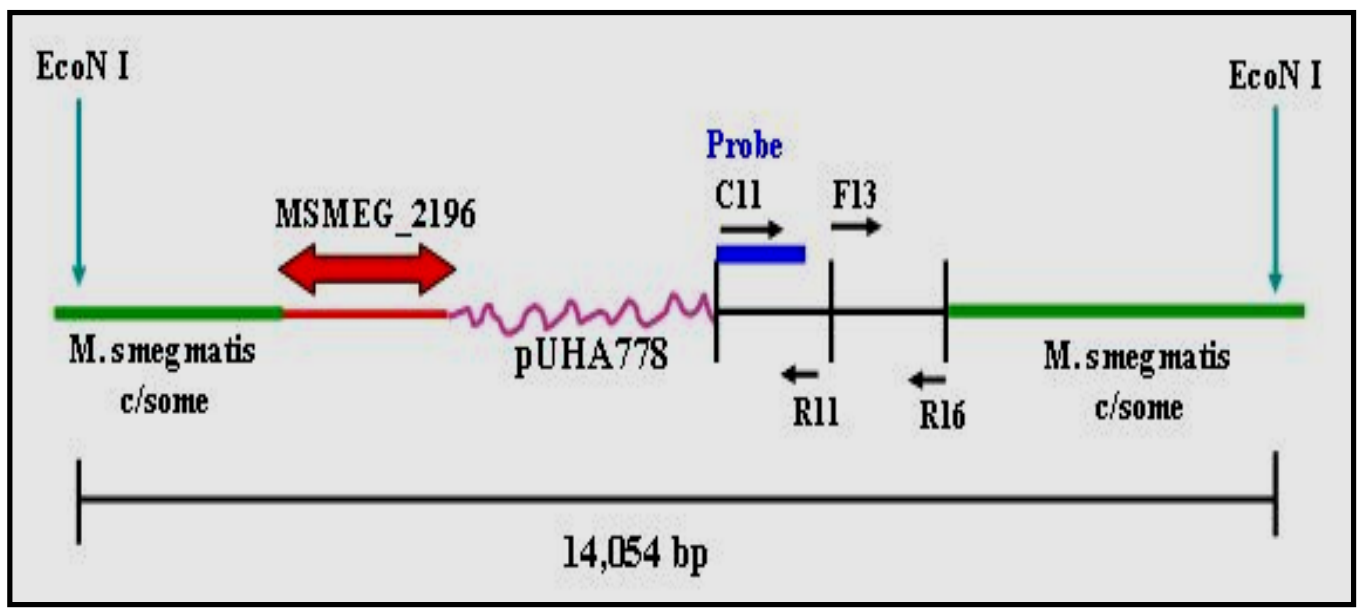

Figure 3.5.16: The expected first cross-over fragment sizes of MSMEG_2196-pUHA778 in Southern

blot. Wild type MSMEG_2196 cut with Econ I enzyme will give a fragment size of 5,398 bp. In the first cross over we expected to see a shift from the wild type to 14,054 bp fragment (this is the total size of wild type MSMEG_2196, the pUHA778 plasmid, MSMEG_2196 overlap fragment [ $1200 \mathrm{bp}$ ], and some of the M. smegmatis chromosome as shown in the above figure. Lines not drawn to scale.

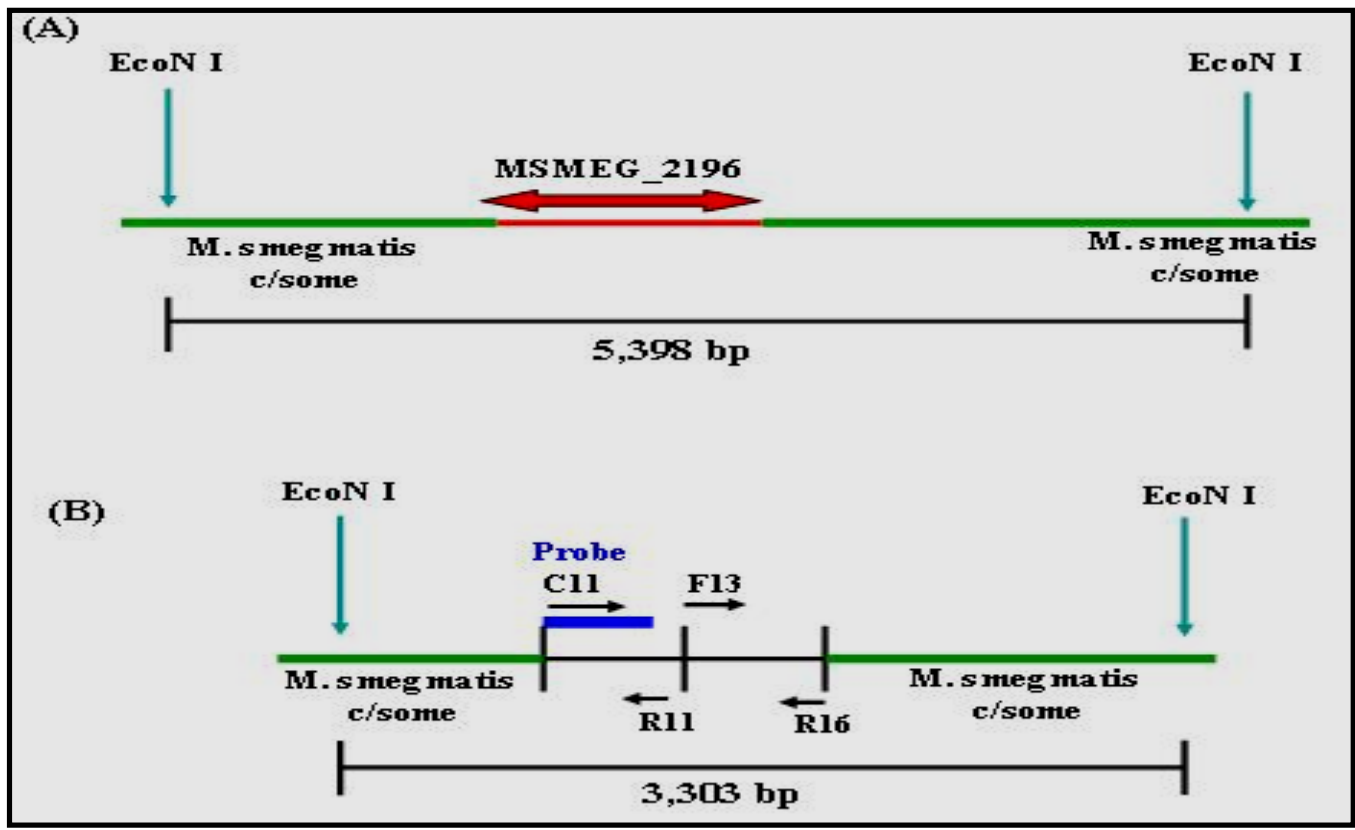

Figure 3.5.17: The expected fragment sizes of second crossover of MSMEG_2196-pUHA778 in

Southern blot analysis. In the second cross-over the pUHA778 plasmid is lost resulting with two

fragments in the Southern blot; either a fragment of 5,398 bp (reversion to wild type) as shown in the above figure (A), or the mutants MSMEG_2196 fragment of 3,303 bp, as shown in the above figure (B). Lines not drawn to scale. 
Chapter 3: Results

\begin{tabular}{|l|l|l|}
\hline Experimental procedures & Mb1389c (M. bovis) & $\begin{array}{l}\text { MSMEG_2196 } \\
\text { (M. smegmatis) }\end{array}$ \\
\hline Upstream flanking region & Completed & Completed \\
\hline Downstream flanking region & Completed & Completed \\
\hline Overlap deletion fragment & Completed & Completed \\
\hline Overlap insertion into pUHA778 & Completed & Completed \\
\hline M. bovis/M. smegmatis mutants & Unfinished & Unfinished \\
\hline
\end{tabular}

Table 3.5.1: Summary of Mb1389c and MSMEG_2196 mutant construction work in $M$. bovis and $M$. smegmatis, respectively. 
Chapter 4: Discussion and future directions

\section{Discussion}

This study looked at the possible role of a cyclic nucleotide, c-di-GMP, in $M$. tuberculosis using $M$. smegmatis as the model strain. The $M$. tuberculosis genome contain 1 gene, annotated as Rv1354c that codes for a GGDEF and EAL domain containing protein. In other bacteria proteins containing GGDEF and EAL domains are known to synthesise and degrade c-di-GMP, respectively $[68,72,75,82,101,122,136$, and 137]. No previous work has been carried out on c-di-GMP in mycobacteria. c-diGMP is ubiquitous in bacteria [25, 26, and 82] (Reviewed in [32], [41], [62] and [109]) and many biosynthesis pathways use c-di-GMP to control responses such as biofilm formation, motility, sessility, and virulence behaviour $[36,37,42,44,49,53,54,55,68$, 74, 76, 77, and 81] (Reviwed in [62]).

This study investigated the growth effect and protein expression that the Rv1354c orthologue (MSMEG_2196) had on M. smegmatis due to the changes made in its expression level under different growth conditions. MSMEG_2196 antisense expressing strain grown in nitrogen-limited condition had a growth defect. The $\mathrm{OD}_{600}$ values at midstationary-phase $(30 \mathrm{hrs})$ of MSMEG_2196 were 0.60 compared to $\mathrm{OD}_{600}$ values of 1.09 for M. smegmatis/pMind (control). Colony forming unit (CFU) measurements further confirmed the growth effect seen in the $\mathrm{OD}_{600}$ of MSMEG_2196 antisense expressing strain. Proteomic analysis of the Ms/p2196 antisense strain demonstrated a difference in protein expression compared to Ms/pMind (control). The construction of Rv1354c mutants in M. bovis (Mb1389c) and M. smegmatis was also initiated. 


\subsection{The effect of modification of MSMEG_2196 expression in $M$. smegmatis}

Antisense and sense fragments of MSMEG_2196 were constructed using the pMind expression vector. The plasmids were transformed into M. smegmatis, this produced $M$. smegmatis strains containing an altered expression of MSMEG_2196: a sense-expressing strain (Ms/p2196 S) and an antisense-expressing strain (Ms/p2196 AS).

To test and observe the effect that expression of MSMEG_2196 sense and antisense had on the growth of M. smegmatis at exponential-phase and stationary-phase, growth curves were conducted using nutrient-rich (LB broth) and nutrient-limited (Hartman deBonts) media. All of the growth assay experiments were performed in triplicate in the presence or absence of tetracycline. Two controls were used; M. smegmatis wild type, and M. smegmatis/pMind, and the two experimental strains examined were Ms/p2196 sense and Ms/p2196 antisense expressing strains.

The growth of all four strains, M. smegmatis WT, Ms/pMind, Ms/p2196 S, and Ms/p2196 AS, in LB broth rich media (in the presence and absence of tetracycline) was not affected. There was no significant difference, outside of standard errors, in specific growth rates and doubling times of the Ms/p2196 S and Ms/p2196 AS strains. There were no significant growth effects (outside of standard errors), observed in the four strains grown either in the carbon, oxygen, or phosphorous nutrient limited HdeB media. A significant growth effect (outside of standard errors) was observed in the Ms/p2196 antisense expressing strain in the nitrogen-limited growth assay. 


\section{Chapter 4: Discussion and future directions}

The Ms/p2196 antisense strain had the most pronounced decrease in $\mathrm{OD}_{600}$ values compared to M. smegmatis WT, Ms/pMind or Ms/p2196 sense. The CFU at 24 and 48 hrs were measured (Table 3.3.6), and the decreased growth of Ms/p2196 antisense strain in the nitrogen-limited media was confirmed by the lower CFU counts. Final $\mathrm{OD}_{600}$ values of all four strains grown in the nitrogen limited condition are displayed in graph 4.1.1. There was a $42 \%$ decrease in the final $\mathrm{OD}_{600}$ readings of Ms/p2196 antisense expressing strain in the presence of tetracycline compared to that of $52 \%$ drop in the Ms/pMind (control). This implies that the down-regulation of MSMEG_2196 in $M$. smegmatis had a significant effect on the cell growth.

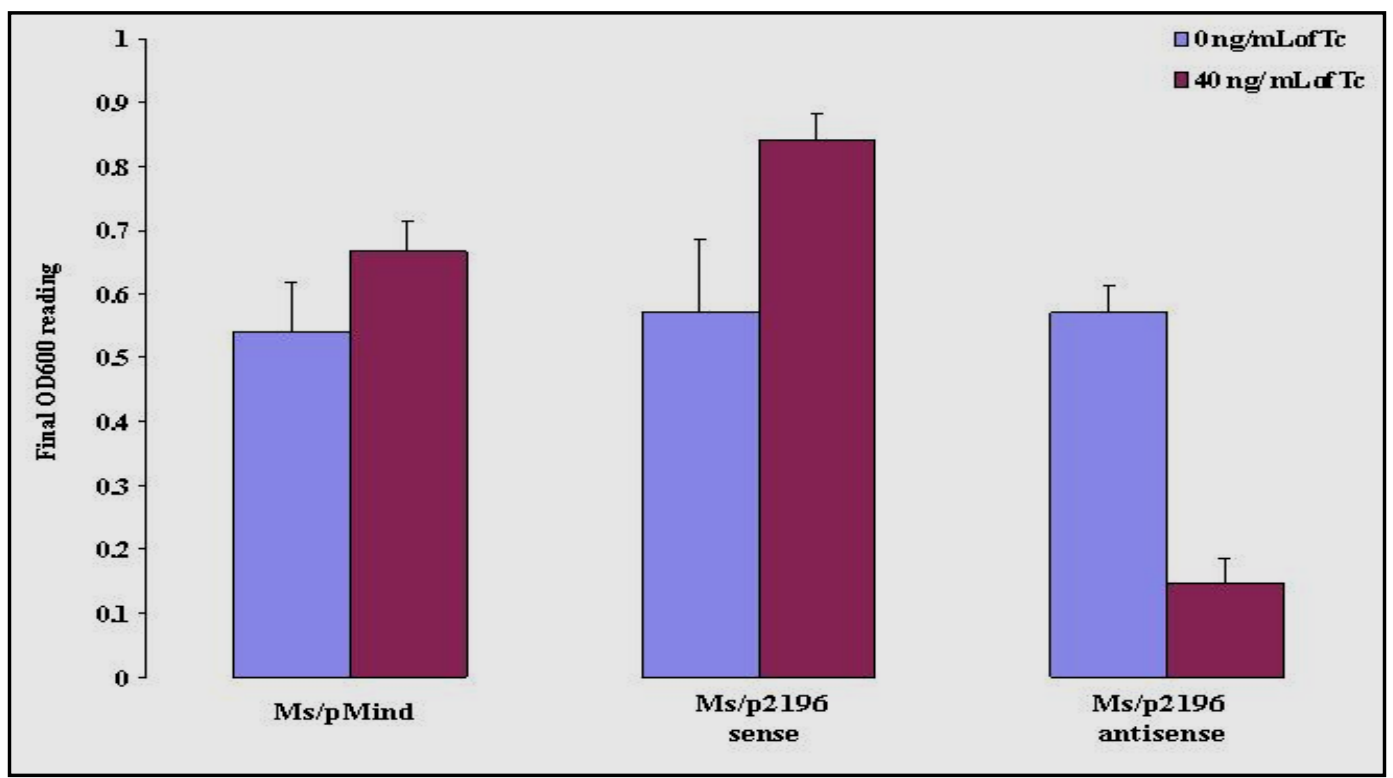

Graph 4.1.1: Final $\mathrm{OD}_{600}$ reading of $\mathbf{N}^{-}$limited growth assay. The final $\mathrm{OD}_{600}$ readings of the three strains: Ms/pMind, Ms/p2196 sense, and Ms/p2196 antisense grown in the presence/absence of Tc. In the presence of Tc Ms/p2196 antisense expressing strain was the most affected compared to the control Ms/pMind, or the Ms/p2196 sense expressing strain. The results represent the mean plus standard error of the final $\mathrm{OD}_{600}$ of the three triplicates for each culture in the presence/absence of Tc. 


\subsection{Proteins identified in Ms/p2196 antisense strain grown in the nitrogen limited-media}

To examine the effect Ms/p2196 antisense expression strain had on protein expression in M. smegmatis, the Ms/p2196 antisense $(\mathrm{Tc}+)$ and Ms/pMind $(\mathrm{Tc}+)$ control cultures were grown in 20 flasks (10 flasks for each strain) containing $50 \mathrm{~mL}$ of HdeB nitrogen-limited media and were harvested at stationary phase (at 1,800 mins). Whole cell protein extraction was carried out on these cultures and were analysed by $2 \mathrm{D}$ gel electrophoresis.

Two proteins that were down-regulated in Ms/p2196 antisense were nitrogen regulatory protein $\mathrm{P}_{\mathrm{II}}$ (spot $\mathrm{O}$ ), down-regulated 13.9 fold, and glutamine synthetase type I (spot K), down-regulated 11.3 fold (Table 4.2.1). Both differentially expressed proteins have possible roles in nitrogen metabolism [63, 85, 98, 99, 104, and 105] (Reviewed in [79]). In M. smegmatis, nitrogen regulatory protein $\mathrm{P}_{\mathrm{II}}$ is annotated as MSMEG_2426 (no gene name has been given in TIGRFAM database) and the M. tuberculosis orthologue is Rv2919 $(g \ln B)$ (Table 4.2.1). M. smegmatis has two genes that are annotated as glutamine synthetase type I; MSMEG_4290 and MSMEG_4294 (both genes are annotated in TIGRFAM databse as $g \ln A$ ), and their orthologues in M. tuberculosis are Rv2220 (glnA1) and Rv2222c ( $g \ln A 2)$, respectively (Table 4.2.1). 
Chapter 4: Discussion and future directions

\begin{tabular}{|l|l|l|l|}
\hline $\begin{array}{l}\text { Proteins identified in } \\
\text { Ms/p2196 AS }\end{array}$ & $\begin{array}{l}\text { Orthologue in } \\
\text { M. tuberculosis }\end{array}$ & $\begin{array}{l}\text { Amino acid } \\
\text { Identities } \\
\text { (\%) }\end{array}$ & E-value \\
\hline $\begin{array}{l}\text { MSMEG_2426 } \\
\left.\text { (Nitrogen regulatory protein } \mathrm{P}_{\text {II }}\right)\end{array}$ & Rv2919 $(g \ln B)$ & 81 & $9.1 \mathrm{e}-44$ \\
\hline $\begin{array}{l}\text { MSMEG_4290 }(g \ln A) \\
\text { MSMEG_4294 }(g \ln A)\end{array}$ & Rv2220 $(g \ln A 1)$ & 83 & $1.6 \mathrm{e}-221$ \\
(Glutamine synthetase typeI) & Rv2222c $(g \ln A 2)$ & 83 & $3.0 \mathrm{e}-205$ \\
\hline
\end{tabular}

Table 4.2.1: The down-regulated nitrogen regulatory proteins identified by $2 \mathrm{D}$ gel analysis of Ms/p2196 antisense expressing strain. This table lists the genes that were down-regulated in the 2D gel analysis of Ms/p2196 antisense grown in nitrogen-limited media. These proteins have a possible role in nitrogen metabolism.

\subsubsection{Nitrogen metabolism in bacteria, and its possible role in MSMEG_2196 in Ms/p2196 antisense strain}

Nitrogen is crucial for amino acid and nucleic acid biosynthesis [4]. Bacteria have evolved a number of ways to acquire nitrogen from sources such as ammonium, which can then be directly incorporated into glutamine and glutamate (Reviewed in [92]). In most bacteria, nitrogen availability is sensed by the glutamine level inside the cell whereby, low intracellular glutamine level signals low nitrogen availability [63]. The synthesis of glutamine is controlled by glutamine synthetase (GS), which is encoded by $g \ln A$ gene $[64,86]$, hence its key function in nitrogen metabolism $[64,86]$. 
The mechanism of nitrogen metabolism has been well documented in E. coli 86, and 105] (Reviewed in [70] and [106]), where post-translational modification is known to be involved in controlling the GS activity during nitrogen assimilation [85, 105]. GS post-translational modification is controlled by proteins such as $\mathrm{P}_{\mathrm{II}}$ (encoded by the gene $g \ln B$ ), and GlnD (an uridylyl transferase/uridylyl removing enzyme that senses the nitrogen state of cell, and changes $P_{\text {II }}$ protein) [63 and 104] (Reviewed in [107]). The GlnB protein is involved in regulating the two-component system proteins $\mathrm{NtrB} / \mathrm{NtrC}$ (involved in nitrogen metabolism) (Reviewed in [92]), as well as controlling the expression of genes involved in nitrogen assimilations such as GS [105] (Reviewed in [92], [93] and [128]).

In the case where intracellular glutamine is reduced (hence low nitrogen), GlnD senses the nitrogen reduction causing uridylylation of the $\mathrm{P}_{\mathrm{II}}$ protein $[60,63,120$, and 126], and the formation of the $\mathrm{P}_{\mathrm{II}}-\mathrm{UMP}$ complex. The $\mathrm{P}_{\mathrm{II}}-\mathrm{UMP}$ complex can not bind to NtrB, which allows NtrB mediated NtrC phosphorylation (NtrC-P), leading to the activation of $g \ln A$ gene translation $[4,61,64$, and 104], and an increase in GS production and glutamine synthesis (Figure 4.2.1). 


\section{Chapter 4: Discussion and future directions}

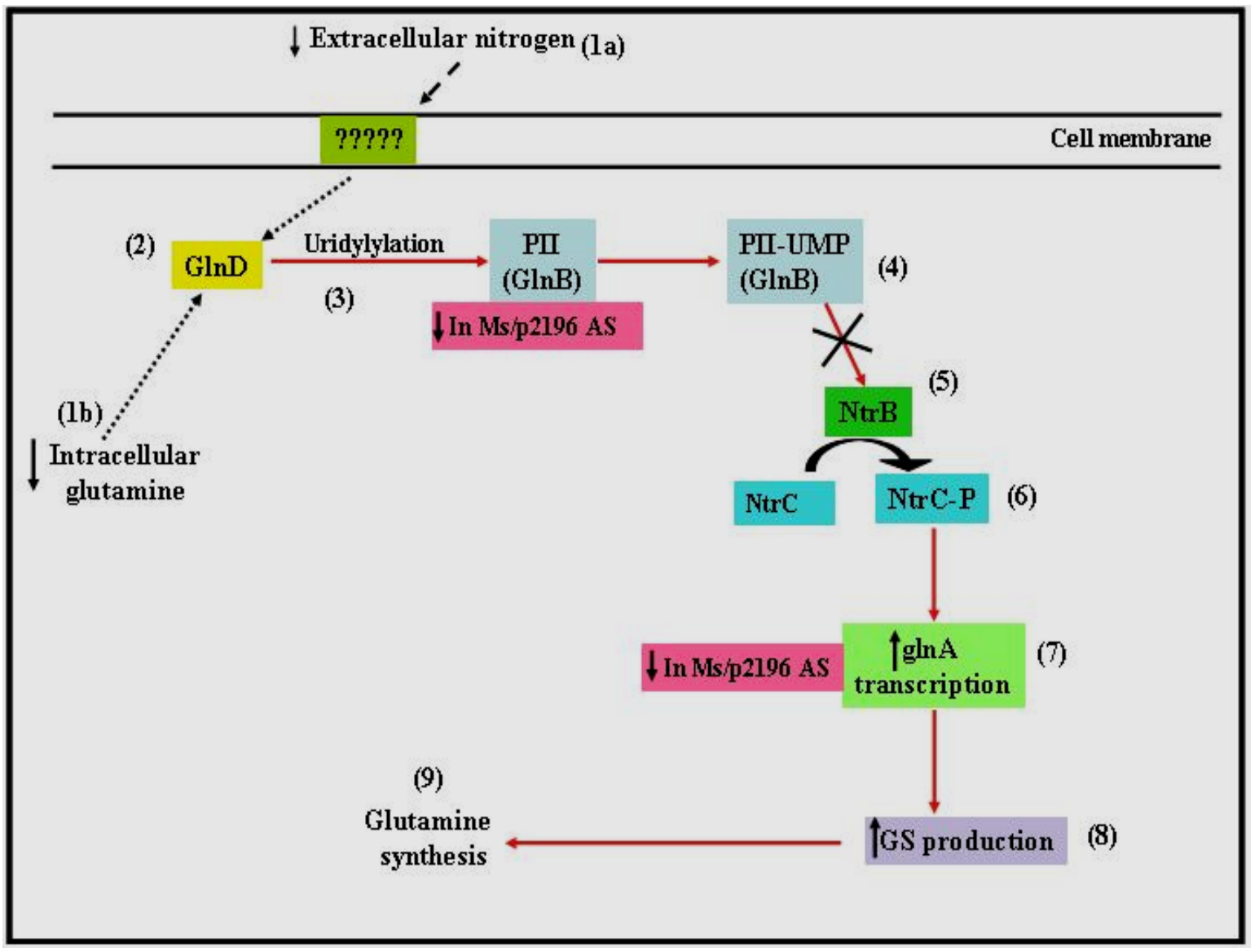

Figure 4.2.1: The mechanism of glutamine production in E. coli under nitrogen starvation. $\mathrm{P}_{\mathrm{II}}$ in $E$. coli is the central regulator of nitrogen metabolism. Signal transduction components and metabolic enzymes also play a major role as shown in this figure. When extracellular nitrogen availability is limited (1a), or the intracellular glutamine concentration decreases (1b), GlnD senses this decrease (2), which then brings about the uridylylation of $\mathrm{P}_{\text {II }}$ protein (3). The uridylylated $\mathrm{P}_{\mathrm{II}}\left(\mathrm{P}_{\mathrm{II}}-\mathrm{UMP}\right)$ (4) can not bind to the two-component system NtrB protein (5), this NtrB protein can then phosphorylate NtrC (6) which activates the transcription of $g \ln A$ gene (7) to increase the production of GS (8), and the synthesis of glutamine (9). The two proteins that were down-regulated in the 2D gel analysis of Ms/p2196 antisense expressing strain are $\mathrm{P}_{\mathrm{II}}$ and $\mathrm{GlnA}$ (pink boxes). 
Chapter 4: Discussion and future directions

It is possible that the down-regulation of MSMEG_2196 in M. smegmatis grown in nitrogen-limited condition could have hindered the production/synthesis of $\mathrm{P}_{\mathrm{II}}$ protein either directly or indirectly, hence its absence in 2D gel analysis of Ms/p2196 antisense culture in this study. This in turn could have interrupted the down-stream signalling pathways involved in glutamine synthetase production and glutamine synthesis (Figure 4.2.2). Although the cells might still be able to sense the depleted nitrogen and glutamine concentration inside and/or outside the cell the cells are not able to synthesise GS. Therefore, the cells will use up all the glutamine and/or ammonia that is available and will become starved for nitrogen. This could explain the Ms/p2196 antisense culture growth behaviour in the presence of tetracycline, where $\mathrm{OD}_{600}$ values never reached an $\mathrm{OD}_{600}$ value similar to that of Ms/pMind control, and after a short while (after $30 \mathrm{hrs}$ ) the $\mathrm{OD}_{600}$ decreased further. However, further experimental procedures, such as RT-PCR or Western blotting needs to be carried out to confirm the reduction of intracellular expression level of MSMEG_2196 in the Ms/p2196 antisense culture. 


\section{Chapter 4: Discussion and future directions}

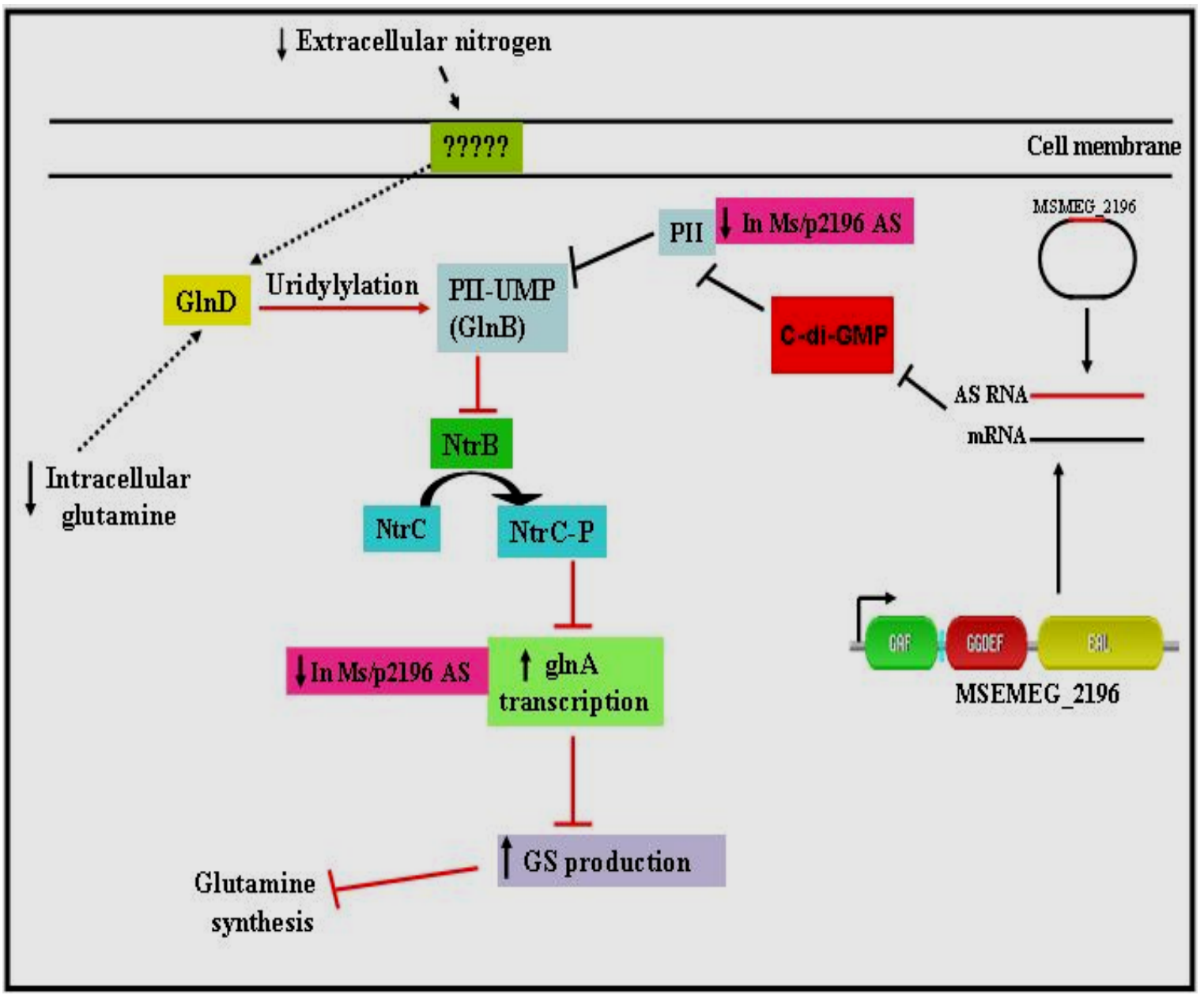

Figure 4.2.2: Proposed c-di-GMP function in M. smegmatis. A hypothetical functional role of c-di-GMP in nitrogen metabolism in M. smegmatis. The decreased-expression of MSMEG_2196 (c-di-GMP), caused by Ms/p2196 antisense expressing strain, could have had an effect on the synthesis of $\mathrm{P}_{\text {II }}$ protein either directly or indirectly. This in turn would have disrupted the down-stream signalling pathway of GS synthesis. The two proteins that were down-regulated in the 2D gel analysis of Ms/p2196 antisense expressing strain were $\mathrm{P}_{\mathrm{II}}$ and GlnA (pink boxes). Dashed lines indicate roles of effectors, solid lines indicate modifications, and blunt lines indicate negative effect. 


\subsubsection{Other down-regulated proteins in Ms/p2196 antisense culture}

The other down-regulated proteins were glutamate 5-kinase, a member of the transcriptional regulator Crp/Fnr family protein, and two 'cold-shock' binding domain proteins. In M. tuberculosis, glutamate 5-kinase (proB), down-regulated 2.8 fold (spot 27, figure 3.4.1), is involved in amino acid biosynthesis [27] similar to glutamine synthetase glnA1 (Rv2220) gene.

Glutamate 5-kinase (G5K) is an enzyme involved in the first step of the proline synthesis pathway [3] (Reviewed in [80]). Proline is synthesised from glutamine, and intracellular proline accumulation exerts a negative feedback effect that causes inhibition of G5K activity [40 and 124] (Reviewed in [80]). In bacteria, proline is used as a source of energy, a source of carbon, and nitrogen, and to protect against osmotic stress $[40,124$, and 127]. The increase in osmolarity, for example during stress, inside bacterial cells causes an increase in proline concentration that has been demonstrated to assist in bacterial growth [10,30,31, and 119]. In the present study, the absence of G5K (not present on the 2D gel of protein identified) in Ms/p2196 antisense expressing strain grown in nitrogen-limited condition could have occurred due to the decreased level of MSMEG_2196 (c-di-GMP) inside the cell, as it was present in the Ms/pMind control atrain.

Members of the transcriptional regulator Crp/Fnr family proteins (a component of cyclic AMP receptor protein [CRP] and fumurate nitrate reductase regulator [FNR] family protein) [1], are involved in mechanisms such as carbon, sulphur, nitrogen metabolism, nitrogen fixation, and expression of virulence genes [46, 73]. In E. coli, CRP proteins are shown to be involved in the expression of many genes and in turn; gene 
expression is controlled by the intracellular concentration of cAMP [43]. The $M$. smegmatis genome contains at least four transcriptional regulatory proteins, MSMEG_0539, MSMEG_6189 (Rv3676 in M. tuberculosis), and MSMEG_2600 (Rv1710 in $M$. tuberculosis). The down-regulation of the transcriptional regulator Crp/Fnr family protein in the Ms/p2196 antisense expressing strain could have, directly or indirectly, been affected by the down-regulations of MSMEG_2196. This is possible because previous studies with $M$. tuberculosis have linked its function with second messenger cAMP [8].

A ‘cold-shock' DNA binding domain protein (CSP), spot 24, was down-regulated 1.5 fold in the Ms/p2196 antisense strain (Figure 3.4.1). In bacteria, this protein is usually expressed when bacterial cells undergo a temperature transition, for example a decrease in temperature from $37{ }^{\circ} \mathrm{C}$ to $15^{\circ} \mathrm{C}$ [102]. This decrease in temperature causes some bacteria, such as E.coli, to undergo a "growth arrest" [67], while other bacteria such as the gram-positive $B$. subtilis, continue to grow in cold temperatures, but at a very slow rate [144]. The expression of cold-shock proteins under decreased temperature helps the cells to cope with the stress by maintaining the cell's transport, replication and energy production, and it helps to maintain proper protein folding, protein biosynthesis and primary metabolism [18, 39, 45, and 67] (reviewed in [33]).

In addition, CSP are involved in other stress conditions. For example, under osmotic and oxidative stress, CSP in E. coli are shown to regulate stress proteins such as OsmY, Dps, ProP and KatG [38]. CSP presence in Ms/pMind (control) grown in the nitrogen limited media under optimum temperature $\left(37^{\circ} \mathrm{C}\right)$ indicates its presence to be of importance for mycobacteria in stress conditions. The down-regulation of a CSP in 
Ms/p2196 antisense expressing strain could have been a direct or indirect result of downregulation of MSMEG_2196 in the cells.

\subsubsection{Up-regulated proteins in Ms/p2196 antisense culture}

The Chaperon protein DnaK, spot A (Table 3.4.1), was up-regulated 1.8 fold. In M. smegmatis it is annotated as MSMEG_0709 and its function is predicted to be a chaperon protein DnaK. MSMEG_0709 orthologue in M. tuberculosis is Rv0350 its function in $M$. tuberculosis is assigned as "DnaK, 70KD heat-shock protein, and chromosome replication" [27]. The M. tuberculosis' genome contains seven genes potentially coding for heat-shock proteins [27, 127]. Like cold shock proteins, the expression of heat-shock proteins (HSP) in bacteria occurs when the cells undergo a change in temperature and/or stress conditions [123] (Reviewed in [83]). The main function of HSPs is to prevent the accumulation and misfolding of proteins, and allow protein refolding and correct protein assembly in both normal and stress conditions [21, 29 and 35] (Reviewed in [52], [59] and 143). It is possible that the up-regulation of chaperon protein DnaK and HSP proteins in the 2D gel analysis of Ms/p2196 antisense expressing strain could have been induced as the cells in the nitrogen-limited condition started to die off, or became stressed.

There were a number of strongly up regulated proteins in Ms/p2196 antisense culture. One of these proteins is geranylgeranyl reductase; spot 10, up regulated 28 fold (Table 3.4.1). The $M$. smegmatis genome contains two geranylgeranyl reductase genes: MSMEG_1028 and MSMEG_2308. In M. smegmatis, the two-geranylgeranyl reductases have a cellular functional role of cofactor biosynthesis; their molecular function is 
Chapter 4: Discussion and future directions

assigned as FAD binding proteins [133]. A study conducted on expression of putative proteins in $M$. tuberculosis under stress condition showed Rv0561c (an orthologue of geranalygeranyl reductase) to be expressed as one of the possible "stress signalling molecule" [34]. It is hypothesised that Rv0561c, along with other stress signalling molecules, can act as a back-up stress signal to sigma factor SigF [34]. M. tuberculosis cells exposed to $\mathrm{NaCl}_{2}$ stress displayed a 12-fold up-regulation of Rv0561c [34]; exposure to methyl viologen (a super-oxide generator) also caused an up-regulation in the expression of Rv0561c [34]. No studies have been conducted with this gene in $M$. smegmatis. The up-regulation of this geranylgeranyl reductase in Ms/p2196 antisense expressing strain could have been caused by the down-regulation of MSMEG_2196, directly or indirectly, or it could have been induced due to the cells becoming stressed in the nitrogen limited condition.

Other strongly up-regulated proteins seen in Ms/p2196 antisense were 50S ribosomal protein L6 (rplF), spot 7, up-regulated 31.4 fold, ribosomal protein L14 (rplN), spot 20/21, up-regulated 32 fold, and ribosomal protein L11 (rplK), spot 5, up-regulated 52.4 fold, (Table 3.4.1). The main functional role of ribosomal proteins is protein synthesis; hence, their expression is crucial to maintain growth of bacterial cells.

The up-regulation of these ribosomal proteins in Ms/p2196 antisense is very interesting as most studies conducted on protein expression of $M$. tuberculosis grown in starvation media, for example oxygen and carbon starvation, have shown that in most cases mycobacterial ribosomal proteins are mainly down-regulated [12, 13, and 58] (Reviewed in [92]). The production of protein in bacterial cells require energy and it is consistent that cells undergoing starvation discontinue protein synthesis [13, 58, and 91]; also, mycobacterial cells in stationary phase do not carry out translation due to the 
possibility of introducing errors in transcription/translation [141] (Reviewed in [140]). Other ribosomal proteins that were slightly up-regulated were ribosomal protein S7, spot 2, up-regulated 13.3 fold, ribosomal protein L10, spot 14, up-regulated 1.15 fold, ribosomal protein L1, spot, 6 up-regulated 1.7 fold, and ribosomal protein S10, spot 23, up-regulated 5.8 fold (Table 3.4.1). It is possible that the up-regulation of all these ribosomal proteins are due to the down-regulation of MSMEG_2196, this could have been affected directly or indirectly in the nitrogen limited condition.

The phenotypic effect in terms of the growth defect observed in the Ms/p2196 antisense strain in nitrogen limited media appears to be specific for nitrogen starvation, as there was no phenotypic growth effect observed in the carbon-, phosphorous-, or oxygenlimited growth media. 2D gel electrophoresis analysis of Ms/p2196 antisense culture grown in nitrogen-limited media has allowed observation of the proteins expressed and/or down-regulated that could be playing role in c-di-GMP related signalling pathways in $M$. smegmatis.

It is clear, from the results presented in this study, that Ms/p2196 antisense had a significant impact on M. smegmatis cell growth compared to Ms/pMind (control), and it is possible that c-di-GMP in mycobacteria could be acting in a similar manner as cAMP having second messenger characteristics that regulate various signalling pathways. 


\subsection{Model of the role of MSMEG_2196 in mycobacterial physiology and virulence behaviour}

The study of Ms/p2196 antisense expressing strain in the nitrogen limited condition has led to a phenotypic effect where cell growth was mostly affected under nitrogen starvation. Further proteomic analysis of Ms/p2196 antisense expressing strain grown in the nitrogen limited condition has shown two down-regulated proteins that are known to be involved in nitrogen synthesis: nitrogen regulatory protein $\mathrm{P}_{\mathrm{II}}(g \ln B)$, and glutamine synthetase type I $(g \ln A)[63,85,98,99,104$, and 105] (Reviewed in [79].

Studies based on nitrogen synthesis in mycobacteria have shown nitrogen regulatory protein $\mathrm{P}_{\mathrm{II}}(\mathrm{G} \ln \mathrm{B})$ to be essential whereby the construction of $g \ln B$ mutant was shown to be lethal [98, 99, and 104]. Study based on the GS gene glnA1 in $M$. tuberculosis is shown to be of importance for virulence [138], whereby glnA1 mutant was shown to be a "glutamine auxotroph" (is not able to synthesise glutamine) and had a decreased ability to divide in infected macrophages and guinea pigs [138]. Although $M$. tuberculosis genome encodes four $g \ln A$ genes $(g \ln A 1-4)$, only $g \ln A 1$ is shown to be involved in the action of glutamine synthetase $[51,138]$. The reduced-expression of nitrogen regulatory protein $\mathrm{P}_{\mathrm{II}}(g \ln B)$ and glutamine synthetase type $\mathrm{I}(g \ln A)$ seen in the 2D gel analysis of Ms/p2196 antisense expressing strain in nitrogen-limited condition could have been caused by the down-regulation of MSMEG_2196, either directly or indirectly. This gives the possibility that MSMEG_2196 could be involved in the signalling pathways of nitrogen metabolism, which in turn is of importance in the virulence behaviour of mycobacteria. 
The other protein seen to be down-regulated in Ms/p2196 antisense expressing strain was the transcriptional regulator $\mathrm{Crp} / \mathrm{Fnr}$ proteins. M. tuberculosis genome contains two transcriptional regulator Crp/Fnr genes: Rv1719 and Rv3676. Studies carried out on Rv3676 mutants in M. tuberculosis have demonstrated that Rv3676 is required for virulence in a mouse models [108] and the growth of these mutants is weakened [108]. Rv3676 mutants are affected in the expression of other genes that could be involved in persistence and reactivation of M. tuberculosis, such as "resuscitation promoting factor proteins" (Rpfs) [90]. Rpfs can activate the growth of the extended stationary phase observed in M. tuberclosis [90]. Little is known about Rv1719.

It is possible that the Ms/p2196 antisense expressing strain could have affected the down-stream signalling pathways involved in multiple mechanisms such as the persistence signalling pathways in mycobacteria, or nitrogen-metabolism and glutamine synthesis which are important in virulence behaviour of mycobacteria, in this case probably under nitrogen starved condition.

\subsection{Future directions}

The starvation growth curves conducted in this study have led to the identification of a phenotypic effect of Ms/p2196 antisense in nitrogen-limited media, and proteomic analysis has given insight into the functional role of the MSMEG_2196 gene in M. smegmatis. Two important experimental approaches need to be conducted before any further analyses on MSMEG_2196 are undertaken, first, to find out if MSMEG_2196 produce c-di-GMP and second, to carry out RT-PCR, or Western blot analysis to test 
Chapter 4: Discussion and future directions

mRNA expression of c-di-GMP in Ms/p2196 antisense. An experimental approach that could be carried out to find if MSMEG_2196 produces cyclic di-GMP is using an expression vector where MSMEG_2196 can be expressed then purified. Nucleotides such as GTP could be added to the purified MSMEG_2196 (cyclic di-GMP is synthesised from 2 GTP molecules). Thin layer chromatography (TLC) could be used to confirm the conversion of MSMEG_2196 into cyclic di-GMP.

If MSMEG_2196 produces cyclic di-GMP, is it affecting the expression of nitrogen regulatory protein $\mathrm{P}_{\mathrm{II}}(\mathrm{G} \ln \mathrm{B})$ and glutamine synthetase type $\mathrm{I}(\mathrm{G} \ln \mathrm{A})$ expression? One experimental approach that could be carried out to answer this question is the construction of two promoters whereby one is inserted in front of the $g \ln B$ gene and the other in front of the $g \ln A$ gene. Cyclic di-GMP could then be added to the Ms/p2196 antisense expressing strain grown in nitrogen-limited condition. 2D gel analysis could then be carried out to see if nitrogen regulatory protein $\mathrm{P}_{\mathrm{II}}$ and glutamine synthetase are expressed and this could be compared to the culture of Ms/p2196 antisense expressing strain grown in nitrogen limited condition in the absence of cyclic di-GMP.

Since, in other bacteria such as $V$. cholerae and Psedumonas, cyclic di-GMP is found to be involved in virulence $[36,49,55$, and 74$]$ another experimental approach that could be carried out is testing the growth of Ms/p2196 antisense expressing strain in varying $\mathrm{pH}$ values and macrophages. Given that c-di-GMP is involved in the biofilm formation and motility of bacteria, Ms/p2196 antisense expressing strain grown in the nitrogen-limited condition could be tested for these functions. The work on constructing Mb1389c (in M. bovis), and MSMEG_2196 (in M. smegmatis) mutants should be resumed. To test the role of cyclic di-GMP in virulence in $M$. bovis, Mb1389c mutants 
Chapter 4: Discussion and future directions

could be tested for pathogenicity of tuberculosis in mice, whereas the cell growth of MSMEG_2196 mutants could be tested in nitrogen-limited conditions.

The Ms/p2196 antisense expressing strain grown in the nitrogen-limited conditions had a significant effect (outside of standard errors) on the growth of $M$. smegmatis.

Proteomic analysis gave a possible role of MSMEG_2196 in M. smegmatis as a cell signalling molecule that could be involved in nitrogen synthesis, of which the latter is shown to be of important in physiology and virulence behaviour of $M$. tuberculosis [57, 115, 122, and 156] (Reviewed in [114]). It is possible that MSMEG_2196 is acting as a second messenger in mycobacteria, such as in the signalling pathways of nitrogen synthesis under nitrogen starvation. This in turn is affecting the down-stream signalling pathways of other proteins such as the transcriptional regulator Crp/Fnr proteins and glutamate 5-kinase. Further analysis of cyclic di-GMP as a second messenger in mycobacteria needs to be carried out, as there is the possibility of it being used as an antiTB drug in tuberculosis. 


\section{Appendices}

\subsection{Plasmids used}

\subsection{1 pUHA778}

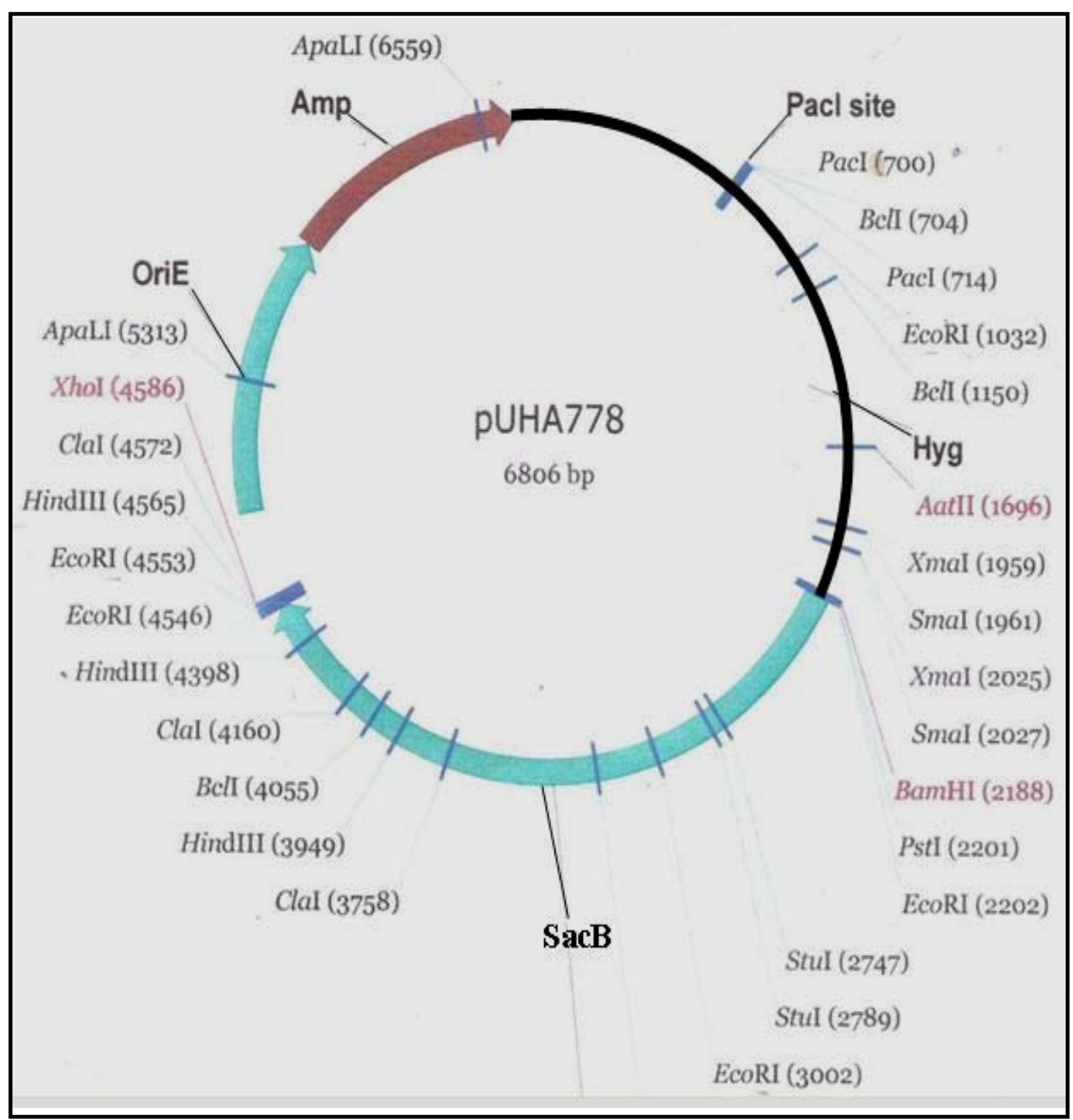




\subsection{2 pMind}

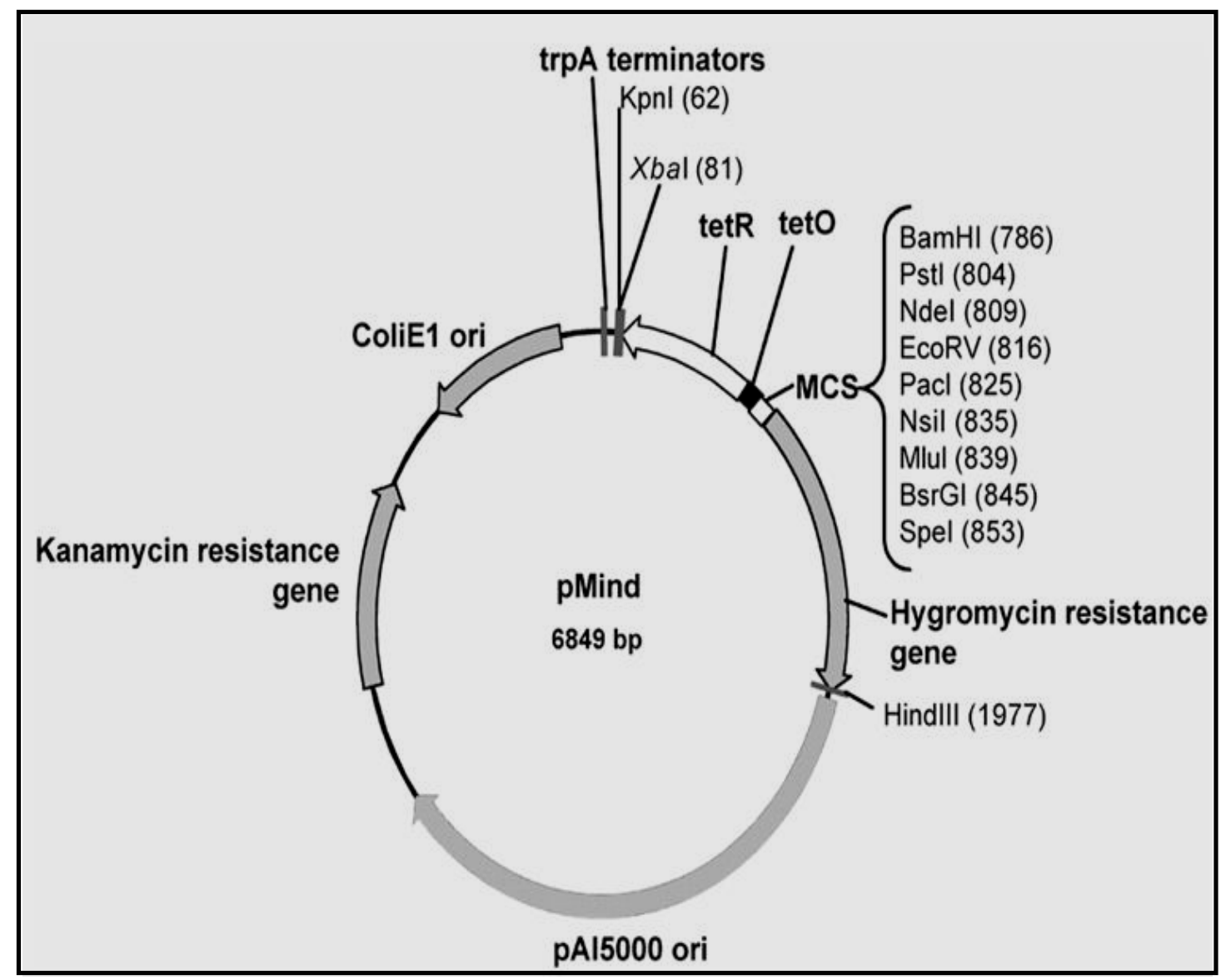




\subsection{Hartman-de Bonts (HdeB) minimal media}

\section{$\underline{100 X \text { metal salts solution }}$}

- $\mathrm{CaCl}_{2} \cdot 2 \mathrm{H}_{2} \mathrm{O} \quad 0.10 \mathrm{~g}$

- $\mathrm{CoCl}_{2} .2 \mathrm{H}_{2} \mathrm{O} \quad 0.04 \mathrm{~g}$

- $\mathrm{CuSO}_{4} .5 \mathrm{H}_{2} \mathrm{O} \quad 0.02 \mathrm{~g}$

- EDTA $1.00 \mathrm{~g}$

- $\mathrm{FeSO}_{4} \cdot 7 \mathrm{H}_{2} \mathrm{O} \quad 0.50 \mathrm{~g}$

- $\mathrm{MgCl}_{2} \cdot 6 \mathrm{H}_{2} \mathrm{O} \quad 10.00 \mathrm{~g}$

- $\mathrm{MnCl}_{2} \cdot 2 \mathrm{H}_{2} \mathrm{O} \quad 0.10 \mathrm{~g}$

- $\mathrm{NaMoO}_{4} \cdot 2 \mathrm{H}_{2} \mathrm{O} \quad 0.02 \mathrm{~g}$

- $\mathrm{ZnSO}_{4} .7 \mathrm{H}_{2} \mathrm{O} \quad 0.20 \mathrm{~g}$

Make up to 1 litre and sterilise by autoclaving

\section{$100 \times$ Nitrogen}

- $\left(\mathrm{NH}_{4}\right)_{2} \mathrm{SO}_{4} \quad 200 \mathrm{~g}$

Make up to 1 litre and sterilise by autoclaving

\section{$100 \times$ Phosphates}

- $\mathrm{K}_{2} \mathrm{HPO}_{4} \quad 155 \mathrm{~g}$

- $\mathrm{NaH}_{2} \mathrm{PO}_{4} \quad 85 \mathrm{~g}$

Make up to 1 litre and sterilise by autoclaving

\section{$\underline{\text { HdeB base }}$}

- $\mathrm{H}_{2} \mathrm{O} \quad 945 \mathrm{ml}$

- $100 \times$ metal salts $\quad 10 \mathrm{ml}$

- $10 \%$ Tween $80 \quad 5 \mathrm{ml}$

Streilise by autoclaving 


\section{Carbon starvation $\left(\mathrm{C}^{-}\right)$broth}

- HdeB base

- 100 x Nitrogen

- $100 \times$ phosphates

- $10 \%$ Glycerol

- Sterile $\mathrm{H}_{2} \mathrm{O}$

\section{Oxygen starvation $\left(\mathrm{O}_{2}^{-}\right)$broth}

- HdeB base

- 100 x Nitrogen

- 100 x Phosphate

- $10 \%$ Glycerol

- Sterile $\mathrm{H}_{2} \mathrm{O}$

\section{$\underline{\text { Nitrogen starvation }\left(\mathrm{N}^{-}\right) \text {broth }}$}

- HdeB base

- 100 x Nitrogen

- $100 \times$ Phosphates

- $10 \%$ Glycerol

- Sterile $\mathrm{H}_{2} \mathrm{O}$

\section{Phosphate starvation ( $\mathrm{P}^{-}$) broth}

- HdeB base

$960 \mathrm{~mL}$

- 100 x Nitrogen

$10 \mathrm{~mL}$

- $100 \times$ Phosphates

- $10 \%$ Glycerol

$1 \mathrm{~mL}$

$20 \mathrm{~mL}$

- $5 \mathrm{M}$ MOPS

- Sterile $\mathrm{H}_{2} \mathrm{O}$
$960 \mathrm{~mL}$

$10 \mathrm{~mL}$

$10 \mathrm{~mL}$

$8 \mathrm{~mL}$

$12 \mathrm{ml}$

$960 \mathrm{~mL}$

$10 \mathrm{~mL}$

$10 \mathrm{~mL}$

$20 \mathrm{~mL}$

$0 \mathrm{~mL}$

$960 \mathrm{~mL}$

$0.1 \mathrm{~mL}$ $10 \mathrm{~mL}$ $20 \mathrm{~mL}$

$9.9 \mathrm{~mL}$
$0 \mathrm{~mL}$ 


\subsection{Preparation and electroporation of Mb1389c-pUHA778 into $M$. bovis WAg203 strain at AgResearch}

\subsubsection{Preparation of Mb1389c-pUHA778 deletion fragment for electroporation}

At AgResearch M. bovis WAg203 strain was used in which the Mb1389cpUHA778 fragment was transformed in. $0.3 \mathrm{~mL}$ of $M$. bovis Wag203 were subcultured into $1 \mathrm{X} 100 \mathrm{~mL}$ of 7H9 Middlebrook broth containing albumin dextrose catalase (ADC) enrichment in a roller bottle and incubated at $37{ }^{\circ} \mathrm{C}$ for up to 1 week. After which cell wall inhibitors (5 mL $30 \%$ glycine, $1 \mathrm{~mL}$ M-fluro-DL-phenylanine [MFP], and $1 \mathrm{~mL} \mathrm{D}$ norleucine [DNL]) were added to the cell culture, and incubate at $37^{\circ} \mathrm{C}$ for 2 days.

Before transformation, the DNA was denatured (to increase transformation efficiency), for gene knock-outs, using alkaline denaturation protocol, briefly; $1-2 \mu \mathrm{g}$ of Mb1389-pUHA778 deletion fragment DNA was diluted to $30 \mu \mathrm{L}$ with sterile distilled water, and $3 \mu \mathrm{L}$ of $2 \mathrm{M} \mathrm{NaOH}$ was added and incubated at room temperature for 5 mins. To this, $5 \mu \mathrm{L}$ of $3 \mathrm{M} \mathrm{NaOAc}$, at $\mathrm{pH} 5$, and $120 \mu \mathrm{L}$ of cold $100 \%$ ethanol was added, mixed and incubated at room temperature for $5 \mathrm{mins}$ then centrifuged at 1,4000 rpm for another 5 mins. The supernatants were then removed and $120 \mu \mathrm{L}$ of $70 \%$ cold ethanol added, and centrifuged once again at 1,4000 rpm for 5 mins. Finally, the supernatants were removed and DNA dried, this DNA is ready for use in electro-transformation, otherwise it could be stored at $4{ }^{\circ} \mathrm{C}$ overnight.

\subsubsection{Electroporation of Mb1389c-pUHA778 into M. bovis WAg203}

The $M$. bovis WAg203 culture in 5.3.1 were transferred into 2x $50 \mathrm{~mL}$ Falcon tubes, $1 \mathrm{~mL}$ of this culture was removed to measure $\mathrm{OD}_{600}\left(\mathrm{OD}_{600}\right.$ of 1.3). The Falcon tubes were centrifuged at $3500 \mathrm{~g}$ at room temperature for 10 mins. The supernatants were discarded, and each pair of pellets were resuspended in a small volume (few $\mathrm{mL}$ ) of room temperature heated $10 \%$ glycerol, and combined in a total volume of $45 \mathrm{ml} \mathrm{10 \%}$

glycerol. A second spin was carried out at 3,500 g for another $10 \mathrm{mins}$, the pellets were resuspended into $45 \mathrm{~mL}$ of $10 \%$ glycerol as previously and a final spin was performed 
after which the pellets were resuspended in $600 \mu \mathrm{L}$ of $10 \%$ glycerol (200 $\mu \mathrm{L} /$ electroporation). $200 \mu \mathrm{L}$ of the resuspended $M$. bovis WAg203 were mixed (by pipetting up and down) with precipitated, dried Mb1389c-pUHA778 DNA. The electroporation cuvettes were pre-warmed in $37{ }^{\circ} \mathrm{C}$ water bath prior to use. Electroporation was carried out at $2.5 \mathrm{kV}, 25 \mathrm{uF}, 1000 \Omega$ using Bio-Rad Gene Pulser electroporator. For control, blank M. bovis WAg203 was used in a similar manner. After electroporation, the content of each cuvette was transferred to a fresh eppendorf tube and incubated at $37{ }^{\circ} \mathrm{C}$ over night to recover. Next day, the electroporated cells were resuspended by pipeting up and down, and plated out onto 7H11 Middlebrook (with ADC enrichment) $\mathrm{Hyg}_{50}$ plates. The plates were incubated at $37{ }^{\circ} \mathrm{C}$ for about 3 weeks, after which colonies were picked and inoculated into $3 \mathrm{~mL}$ of $7 \mathrm{H} 9$ Middlebrook $\mathrm{Hyg}_{50}$ broth (containing ADC enrichment). The cell cultures were heat killed at AgResearch before entering Victoria University PC2 laboratories.

\subsection{Southern blot}

1. Run a thick gel, $180-200 \mathrm{~mL}$

- $0.8 \%-1.0 \%$ agarose gel

- Run gel at a very slow rate, for example $30 \mathrm{~V}$ for $6 \mathrm{hrs}$, or $60 \mathrm{~V}$ for $3 \mathrm{hrs}$.

2. Cut 1 piece of Hybond membrane and 3 pieces of Whatman $3 \mathrm{~mm}$ filter paper to exact size of gel.

3. Prepare a plastic container as reservoir, a glass plate to sit gel on, 3 pieces of Whatman $3 \mathrm{~mm}$ filter paper as bridge, 2 plastic containers to wash gel, and the following solutions:

A. Depurination wash

- $11 \mathrm{~mL}$ of conc. $\mathrm{HCl}$

- $\quad$ Up to $500 \mathrm{~mL}$ with $\mathrm{dH} 2 \mathrm{O}$ 
- Or $500 \mathrm{~mL}$ of $0.25 \mathrm{M} \mathrm{HCl}$ acid

B. Denaturation wash

- $43.83 \mathrm{~g}$ of $\mathrm{NaCl}$

- $10.0 \mathrm{~g}$ of $\mathrm{NaOH}$ pellets

- $\quad$ Up to $500 \mathrm{~mL}$ with distilled water (make up fresh each time)

C. Neutralization wash

- $87.66 \mathrm{~g}$ of $\mathrm{NaCl}$

- $60.56 \mathrm{~g}$ of Tris

- $4 \mathrm{~mL}$ of $0.5 \mathrm{M}$ EDTA $\mathrm{pH} 8.0$

- Dissolve in $800 \mathrm{~mL} \mathrm{dH2O}$, adjust $\mathrm{pH}$ to 7.2 with conc. $\mathrm{HCl}$ up to $1 \mathrm{~L}$ with $\mathrm{dH} 2 \mathrm{O}$

D. $6 \mathrm{X} \mathrm{SSC}$

- 1 L of 6X SSC from 20X SS

\section{Method}

\section{Pre-treatment of gel}

- Put gel in first container for washing gel. Add $500 \mathrm{~mL}$ of depurination wash, rock for 15 mins at low speed

- Transfer gel into second plastic container, rinse gel with distilled water. Wash first plastic container with distilled water, return gel to first container, and add $500 \mathrm{~mL}$ denaturation wash. Rock for $45 \mathrm{~min}$ at low speed.

- Remove gel from denaturation wash into a rinsed second container, and rinse gel with $\mathrm{dH} 2 \mathrm{O}$. Wash first plastic container with $\mathrm{dH} 2 \mathrm{O}$.

- Transfer gel back to first container and add $500 \mathrm{~mL}$ neutralization wash. Rock for $15 \mathrm{~min}$ at low speed. Remove gel and repeat neutralization wash with fresh solution for $15 \mathrm{~min}$. 
- Pre-wet the filter paper and nylon membrane in 6X SSC solution, and pour the rest of the solution into the reservoir

- Place glass plate on the reservoir container and set up re-wetted bridge. Make sure ends of the bridge are in contact wit the SSC solution in the reservoir.

- Carefully slide the gel onto the filter paper bridge, with bottom of gel facing upwards.

- With a $10 \mathrm{~mL}$ clean disposable pipette, gently roll along surface of gel to remove any air bubbles between gel and the filter paper.

- Using forceps lay pre-wetted nylon membrane on top the gel. Roll gently with pipette to remove air bubbles between membrane and gel. Cut one corner of membrane and gel and note orientation.

- Lay pre-wetted $3 \mathrm{~mm}$ filter paper (one by one) on the membrane with forceps, and remove bubbles between layers before putting next layer on.

- Cover the setup with glad wrap to prevent evaporation of buffer. Cut and remove glad wrap covering gel area.

- Place 1 pack of paper towels on top of filter paper, and put weight $(0.5-1 \mathrm{~kg})$ on paper towels. Leave overnight for around $18 \mathrm{hrs}$.

- Next morning, dissemble the set up, place the nylon membrane into a folder made from blotting or filter paper. Wrap folder with aluminium foil and bake in $80^{\circ} \mathrm{C}$ oven for 2 hrs. Alternatively, wrap the membrane in glad wrap and expose it to UV for 1 - 2 mins on each side. 


\section{Hybridization}

- Prepare $25 \mathrm{~mL}$ of hybridization solution (this volume is for small membrane):

\begin{tabular}{|l|l|}
\hline Ingredient & Volume \\
\hline 20X SSC & $7.5 \mathrm{~mL}$ \\
\hline $50 X$ denhardt's solution & $2.5 \mathrm{~mL}$ \\
\hline $10 \%$ SDS & $1.25 \mathrm{~mL}$ \\
\hline Salmon sperm DNA $(9.1 \mathrm{mg} / \mathrm{mL})$ & $0.27 \mathrm{~mL}$ \\
\hline SDW & $13.48 \mathrm{~mL}$ \\
\hline Total volume & $\mathbf{2 5 . 0 0} \mathbf{~ m L}$ \\
\hline
\end{tabular}

- Prehybridize membrane in hybridization solution in Hybaid oven set at $60{ }^{\circ} \mathrm{C}$ for 2 hrs.

- While membrane is prehybridizing, prepare probe.

\section{Probe preparation}

- Dilute template DNA (Mb1389 F4R4 and/or MbF2R4-pGEM-T easy) for probe making to $5 \mathrm{ng} / \mu \mathrm{L}$ (I added $10 \mu \mathrm{L}$ of my template), and prepare a mixture of $\mathrm{dATP} / \mathrm{dTTP} / \mathrm{dGTP}$ at $5 \mathrm{mM}$ each.

- $\quad$ Mix $10 \mu \mathrm{L}$ of DNA template with $75 \mathrm{ng}$ of random primers (about $3 \mu \mathrm{L}$ ).

- Boil for 5 mins and put in ice immediately for 5 mins.

- Add $5 \mu \mathrm{L}$ of 10X NEBuffer 2, $2 \mu \mathrm{L}$ of $5 \mathrm{mM} \mathrm{dATP/dTTP/dGTP} \mathrm{mixture,} 2 \mu \mathrm{L}$ of 5 $\mathrm{U} / \mu \mathrm{L}$ Klenow exo- enzymes, and $23 \mu \mathrm{L}$ of SDW. In the hot room (radioactive room), add $4 \mu \mathrm{L}$ of ${ }^{32} \mathrm{P}-\mathrm{dCTP}$ to the mixture (the total volume of this mixture is $50 \mu \mathrm{L}$ ).

- Incubate the mixture for $1 \mathrm{hr}$ at $37^{\circ} \mathrm{C}$ 
- Boil probe $\left(95-100{ }^{\circ} \mathrm{C}\right)$ for 5 mins, ice immediately for 5 mins, and add to prehyb. Take care not to add probe directly onto membrane.

- Hybridize membranes in Hybaid oven at $60^{\circ} \mathrm{C}$ overnight.

- Next morning, rinse membrane in $2 \mathrm{X} \mathrm{SSC} / 0.5 \% \mathrm{SDS}$ at room temperature for 5 mins, followed by $2 \mathrm{X} \mathrm{SSC} / 0.1 \% \mathrm{SDS}$ for $15 \mathrm{mins}$ at room temperature. Replace the solution wit fresh $0.1 \mathrm{XSC} / 0.5 \% \mathrm{SDS}$, and incubate for $30 \mathrm{mins}$ at $65^{\circ} \mathrm{C}$. After the incubation, rinse membrane briefly with $0.1 \mathrm{X}$ SSC.

- Dry membrane briefly (not completely dry) with blotting paper. Wrap membrane with glad wrap.

- Place membranes into cassette and put in a piece of film in the darkroom.

\subsection{Gene alignments of Rv1354c against Mb1389c and MSMEG_2196}

\subsubsection{Rv1354c sequence alignment against Mb1389c}

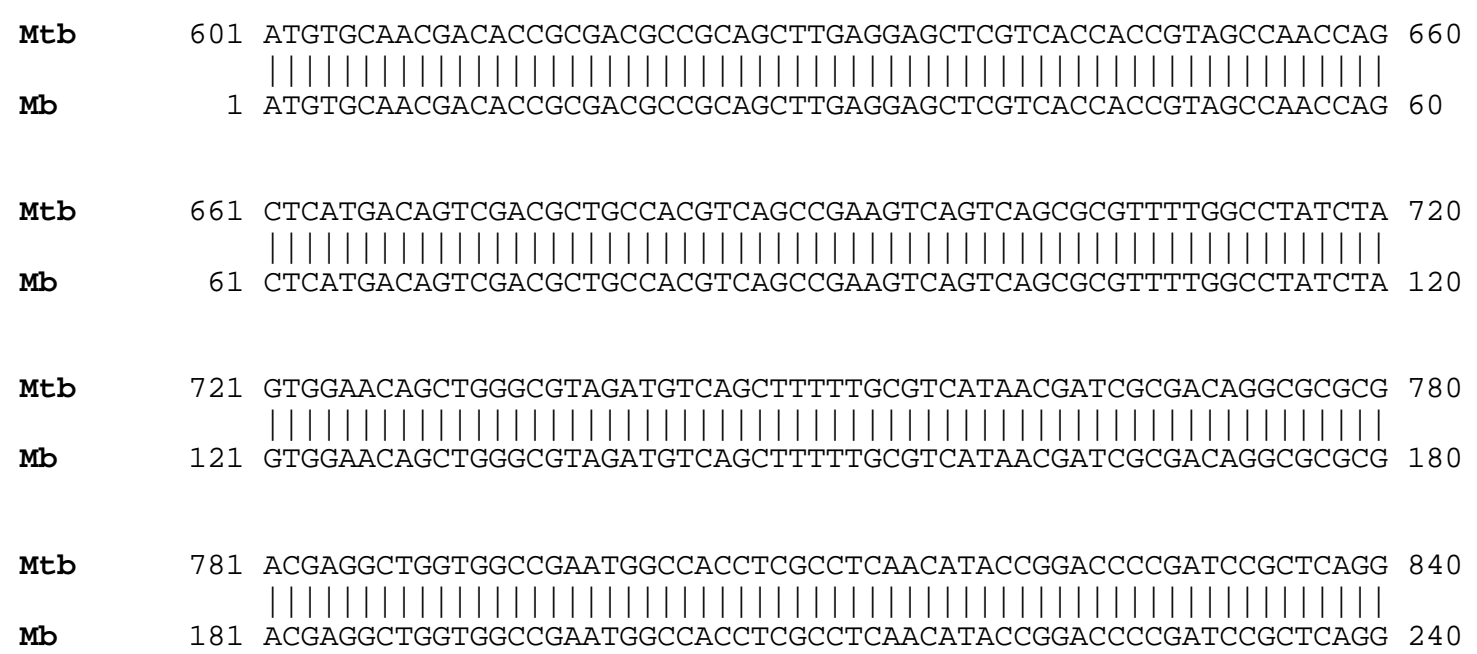




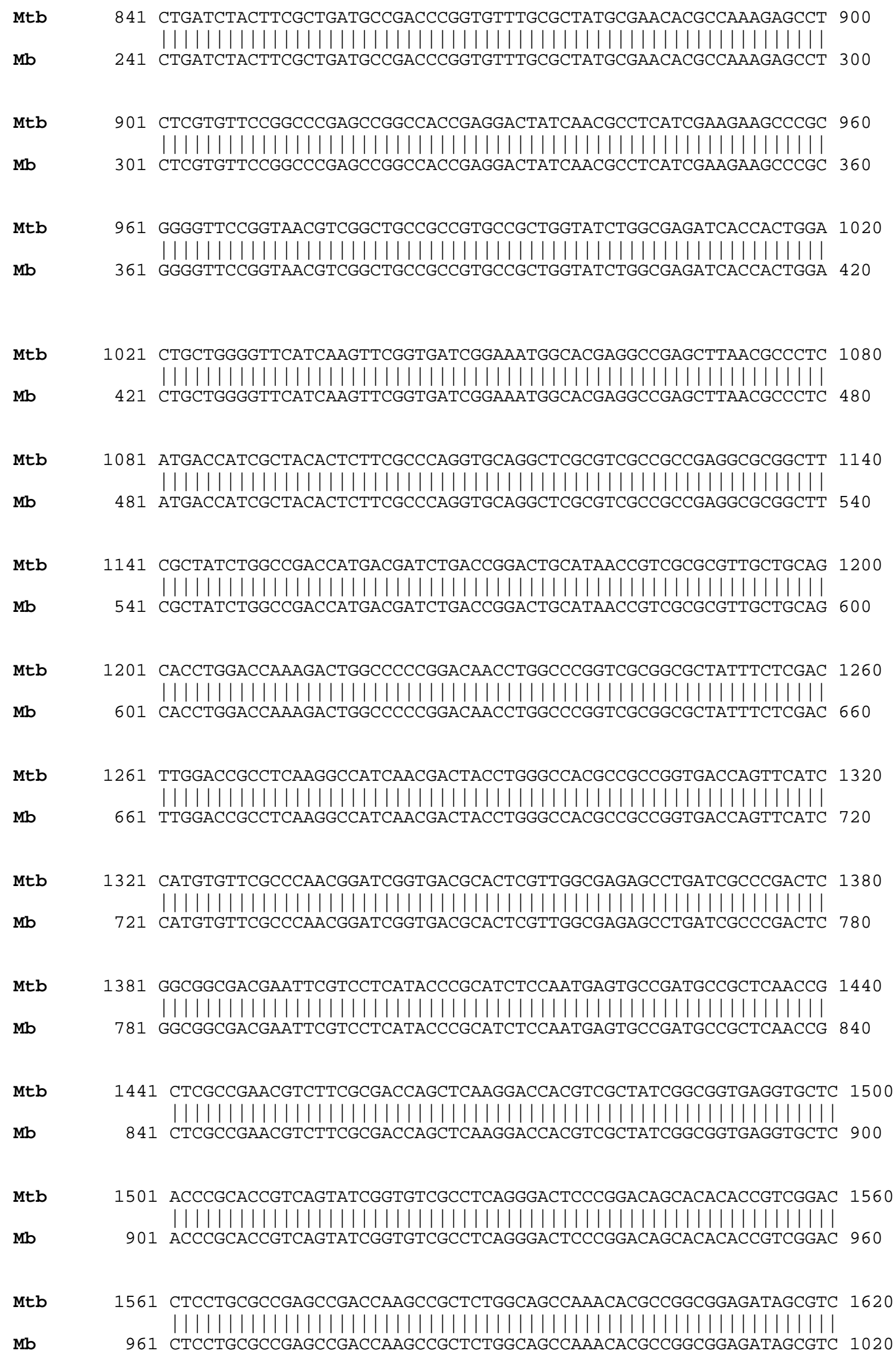




\begin{tabular}{|c|c|c|c|}
\hline Mtb & $\begin{array}{l}1621 \\
1021\end{array}$ & $\begin{array}{l}\text { GCGATTTTCACCGCGGACATGTCGGTCAGCGGCGAACTGCGCAACGATATTGAACTACAC } \\
|||||||||||||||||||||||||||||||||||||||||||||||||||||||||||||||||||||||||||||||||| l \mid \\
\text { GCGATTTTCACCGCGGACATGTCGGTCAGCGGCGACTGCGCAACGATATTGAACTACAC }\end{array}$ & $\begin{array}{l}1680 \\
1080\end{array}$ \\
\hline Mb & 1681 & $\begin{array}{l}\text { CTTCGACGTGGTATCGAATCCGACGCCCTTCGCCTGGTCTACCTACCCGAGGTCGACCTA } \\
|||||||||||||||||||||||||||||||||||||||||||||||||||||||||||||||||||||||||||||||||||||||| l \mid \\
\text { CTTCGACGTGGTATCGAATCCGACGCCCTTCGCCTGGTCTACCTACCCGAGGTCGACCTA }\end{array}$ & $\begin{array}{l}1740 \\
1140\end{array}$ \\
\hline Mb & $\begin{array}{l}1741 \\
1141\end{array}$ & $\begin{array}{l}\text { CGGACCGGCGACATTGTCGGGACCGAGGCATTGGTCCGGTGGCAGCACCCCACCCGTGGG } \\
|||||||||||||||||||||||||||||||||||||||||||||||||||||||||||||||||| \\
\text { CGGACCGGCGACATTGTCGGGACCGAGGCATTGGTCCGGTGGCAGCACCCCACCCGTGGG }\end{array}$ & $\begin{array}{l}1800 \\
1200\end{array}$ \\
\hline Mtb & $\begin{array}{l}1801 \\
1201\end{array}$ & 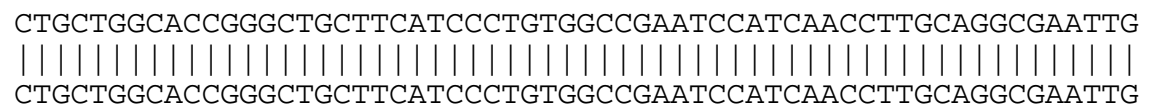 & $\begin{array}{l}1860 \\
1260\end{array}$ \\
\hline Mtb & $\begin{array}{l}1861 \\
1261\end{array}$ & $\begin{array}{l}\text { GATAGATGGGTGCTGCGGAGGGCCTGCAATGAATTCTCCGAGTGGCAGTCAGCCGGTTTG } \\
\text { || || |||||||||||||||||||||||||||||||||||||||||||||||||||||||||||||||||||||||||||||||||| } \\
\text { GATAGATGGGTGCTGCGGAGGGCTGCAATGAATTCTCCGAGTGGCAGTCAGCCGGTTT }\end{array}$ & $\begin{array}{l}1920 \\
1320\end{array}$ \\
\hline Mtb & $\begin{array}{l}1921 \\
1321\end{array}$ & $\begin{array}{l}\text { GGCCACGACGCGCTGCTGCGTATCAACGTCTCAGCTGGACAGCTGGTGACGGGCGGGTTT } \\
|||||||||||||||||||||||||||||||||||||||||||||||||||||||||||||||||| \\
\text { GGCCACGACGCGCTGCTGCGTATCAACGTCTCAGCTGGACAGCTGGTGACGGGCGGGTT }\end{array}$ & $\begin{array}{l}1980 \\
1380\end{array}$ \\
\hline Mtb & $\begin{array}{l}1981 \\
1381\end{array}$ & $\begin{array}{l}\text { GTTGACTTCGTCGCAGACACGATCGGCCAGCACGGTCTGGACGCCTCGTCCGTGTGTTTG } \\
\text { || || |||||||||||||||||||||||||||||||||||||||||||||||||||||||||||||||||||||||||| } \\
\text { GTTGACTTCGTCGCAGACACGATCGGCCAGCACGGTCTGGACGCCTCGTCCGTGTGTTG }\end{array}$ & $\begin{array}{l}2040 \\
1440\end{array}$ \\
\hline Mtb & $\begin{array}{l}2041 \\
1441\end{array}$ & $\begin{array}{l}\text { GAAATCACCGAAAACGTTGTGGTGCAAGACCTACATACCGCCAGAGCCACCCTGGCTCGA } \\
|||||||||||||||||||||||||||||||||||||||||||||||||||||||||||||||||||| \\
\text { GAAATCACCGAAAACGTTGTGGTGCAAGACCTACATACCGCCAGAGCCACCCTGGCTCGA }\end{array}$ & $\begin{array}{l}2100 \\
1500\end{array}$ \\
\hline Mtb & $\begin{array}{l}2101 \\
1501\end{array}$ & $\begin{array}{l}\text { CTCAAAGAAGTCGGCGTTCACATCGCTATCGACGATTTCGGCACCGGCTATAGCGCCATA } \\
|||||||||||||||||||||||||||||||||||||||||||||||||||||||||||||||| \mid \\
\text { CTCAAAGAAGTCGGCGTTCACATCGCTATCGACGATTTCGGCACCGGCTATAGCGCCATA }\end{array}$ & 2160 \\
\hline Mb & 2161 & $\begin{array}{l}\text { TCACTGTTGCAGACGCTACCGATCGACACGCTCAAGATCGACAAAACATTCGTGCGGCAA } \\
|||||||||||||||||||||||||||||||||||||||||||||||||||||||||||||||||||||||||||||||||||||| l|l| \\
\text { TCACTGTTGCAGACGCTACCGATCGACACGCTCAAGATCGACAAAACATTCGTGCGGCAA }\end{array}$ & $\begin{array}{l}2220 \\
1620\end{array}$ \\
\hline Mtb & $\begin{array}{r}222 \\
1621\end{array}$ & $\begin{array}{l}\text { CTCGGAACCAACACTAGCGATCTGGTCATTGTGCGCGGCATCATGACACTCGCCGAAGGC } \\
|||||||||||||||||||||||||||||||||||||||||||||||||||||||||||||||||| \\
\text { CTCGGAACCAACACTAGCGATCTGGTCATTGTGCGCGGCATCATGACACTCGCCGAAGGC }\end{array}$ & $\begin{array}{l}2280 \\
1680\end{array}$ \\
\hline Mtb & $\begin{array}{l}2281 \\
1681\end{array}$ & $\begin{array}{l}\text { TTCCAACTCGATGTAGTAGCCGAAGGCGTCGAGACCGAGGCTGCCGCCAGAATTCTATTG } \\
\text { || || || || ||||||||||||||||||||||||||||||||||||||||||||||||||||||||||||||||||||||| } \\
\text { TTCCAACTCGATGTAGTAGCCGAAGGCGTCGAGACCGAGGCTGCCGCCAGAATTCTATTG }\end{array}$ & $\begin{array}{l}2340 \\
1740\end{array}$ \\
\hline Mtb & 2341 & 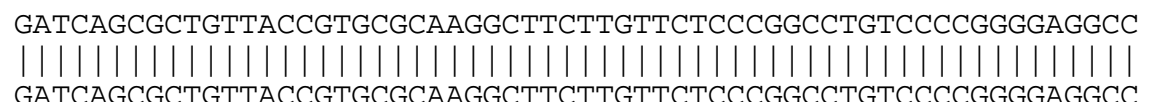 & $\begin{array}{l}2400 \\
1800\end{array}$ \\
\hline
\end{tabular}




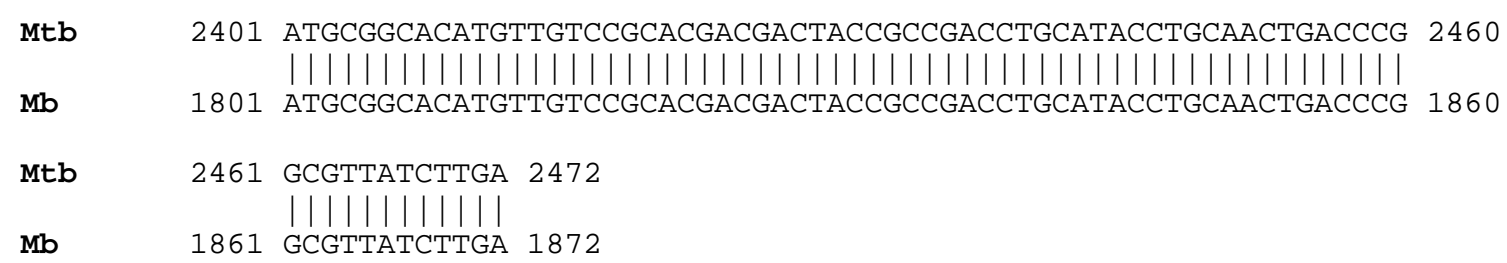

\subsubsection{Rv1354c sequence alignment against MSMEG_2196}

Mtb 622 CCGC-AGCTTG-AGGAGCTCGTCACCACCGTAGCCAACCAGCTCATGACAGTCGACGCTG 679

||| ||| || | | ||| ||||| |||| |||| ||||||||| ||||||| || |

5 CCGAGAGCCTGGACGTGCTGGTCACGTCCGTCGCCACTCAGCTCATGGCAGTCGAGGCGG 64

Mtb 680 CCACGTCAGCCGAagtCAGTCAGCGCGTTTTGGCCTATCTAGTGGAACAGCTGGGCGTAG 739

M.smeg 65 CCACCTCGgTGTCGgTCAGCCAGCAGGTGCTCGCCGAACTGGTGTCGTTCTTCGATGTCG 124

Mtb 740 ATgTCAgCTTTTTgCgtCATAACGATCGCGACAGgCGCGCGACGAGgCTgGTgGCCGAAT 799 |||| ||||| |||| || ||||| | | | | ||| |||||||| || ||||| |

M.smeg 125 ATGTGAGCTTCCTGCGCCACAACGACCACCGCGCCCACGCCACGAGGCTCGTCGCCGAGT 184

Mtb

800 GGCCACCTCGCCTCAACATAC - CGGACCCCGATCCGCTCAGGC - TGATCTACTTCGCTGA 857 |||| || || | | || | | | |||| ||| || | | | || ||||||| ||

M.smeg 185 GGCCGCCGCGTCCGGTCG-ACGCCGCCACCGACCCGATC-GCCGTCGTCCACTTCGCCGA 242

Mtb

858 TgCCGACCCGgTGTTTGCGCTATGCGAACACGCCAAAGAGCCTCTCG-TGTTCCGGCCCG 916

M. smeg

||||||||||||||| | |||||| ||| || || | || ||||||||||||

243 TGCCGACCCGGTGTTCGCCATGGCCGAACACCTCAAGGAACC-CGCGGTGTTCCGGCCCG 301

Mtb 917 AGCCGGCCACCGAGGACTATCAACGCCTCATCGAAGAAGCCCGCGGGGTTCCGGTAACGT 976

M.smeg 302 AACCGCTCTACGACGACTATCAGCGCACCATCGAGGCAGGCCGGCACATCCCCGCCACGT 361

Mtb 977 CGGCTGCCGC-CGTGCCGCTGGT-ATCTGGCGAGATCACCACTGGACTGCTGGGGTTCAT 1034

|| || || ||| |||||| | | | ||||| |||| || || ||||||| ||| |

M.smeg 362 CGA-TGGCGTGCGTCCCGCTGCTCAGC-GGCGACGTCACGACCGGTGTGCTGGGCTTCGT 419

Mtb 1035 CAAGTTCGgTGATCGgAAATGGCACGAGgCCGAGCTTAACGCCCTCATGACCATCGCTAC 1094

M.smeg 420 CAAGTTCGGCGACCGCGAGTGGCTGCCCGCAGAACTCAACGCGCTCAAGGCGATCGCGTC 479

Mtb 1095 ACTCTTCGCCCAGGTGCAGGCTCGCGTCGCCGCCGAGgCGCGGCTTCGCTATCTGGCCGA 1154

M.smeg 480 GTTGTTCGCGCAGGTGCAGGCCCGGATCGAGGCCGAGGAACGCCTGCGTTACCTGGCCGA 539

Mtb 1155 CCATGACGATCTGACCGGACTGCATAACCGTCGCGCGTTGCTGCAGCACCTGGACCAAAG 1214

||| || | |||||||| ||| | ||||||||||| || || ||||||||| ||

M.smeg 540 CCACGATCACCTGACCGGCCTGTACAACCGTCGCGCCCTGATGGCGCACCTGGAAGCGAG 599 


\begin{abstract}
Mtb 1215 ACTgGCCCCCGgACAACCTGgCCCGgTCGCGgCGCTATTTCTCGACTTGGACCGCCTCAA 1274
||| || || || ||||||| || || |||||||||| | | || |||||| |||||||| || ||

M. smeg $60 \odot$ ACTCGCTCCGGGACAACCCGGTCCGGTCGCGGTGATGTTCTTCGACCTGGACCGGCTGAA 659

Mtb 1275 GGCCATCAACGACTACCTGGGCCACGCCGCCGGTGACCAGTTCATCCATGTGTTCGCCCA 1334

M.smeg 660 GGCGATCAACGACTACCTGGGTCACACCGCGGGTGACGCGTTCATCAGCATCCTCGCGCA 719

Mtb 1335 ACGGATCGgTGACGCACT--CGTTGGCGAGAGCCTGATCGCCCGACTCGgCGgCGACGAA 1392

|| | | | ||| | || | || ||||| || || ||||||||||||||

M.smeg 720 GCG-A-CTGCAACGCGGTGACGACGCTCCGAAGCTGATAGCGCGCCTCGGCGGCGACGAG 777

Mtb 1393 TTCGTCCTCATACCCGCATCTCCAATGAGTGCCG-ATGCCGCTCAAC-CGCTCGCCGAAC 1450

M.smeg 778 TTCGTCGTCGTCCCCGACGATCCCATGTC-GCTGGATGAGGC-CACCGCGCTGGCCTACC 835

Mtb 1451 GTCTTCGCGACCA-GCTCAAGGACCACGTCGCTATCGGCGGTGAGGTGCTCACCCGCACC 1509

M.smeg 836 GGCTGCA-GTCGGTGCTGCGCGAACGCGTCACCGTCGACGGTGAGATGCTCACTCGAACC 894

Mtb 1510 GTCAGTATCGgTGTCGCCTCAGGGACTCCCGGACAGCACACACCGTCGGACCTCCTGCGC 1569

M.smeg 895 GTGAGCATCGGTATCGCACAAGGCATTCCGGGTAAGGATTCAACGTCCGACGTGCTCAAC 954
\end{abstract}

Mtb 1570 CGAGCCGACCAAGCCGCTCTgGCAGCCAAACACGCCGgCGGAGATAGCGTCGCGATTTTC 1629

M.smeg 955 CGGGCCGACCACGCGGTATTGACGGCCAAGGGTTCCGGCGGCAACCGCGTCGCGGTCTTC 1014

Mtb 1630 ACCGCGgACATGTCGgTCAGCGg-CGAACTGCGCAACGATATTGAACTACACCTTC-GA- 1686

M.smeg 1015 TCCGACGCGATGGCCATG-GAGATCGACTTCCGCAACGACATCGAACTGCACCTGCAGAG 1073

Mtb 1687 CGTGgTATCGAATCCGACGCCCTTCGCCTGGTCTACCTACCCGAGgTCGACCTACGgACC 1746

M.smeg 1074 CGTG--ATCGAAGGCGGCGCACTGGTGCTGCACTACCTGCCGGAAATCGACATGCGCACC 1131

Mtb 1747 GgCGACATTGTCGgGACCGAGGCATTGGTCCGGTGGCAGCACCCCACCCGTGGGCTGCTG 1806

M.smeg 1132 GGTGAGGTGCTGGCGGCAGAAGCGCTGGTCCGCTGGGAGCATCCCACCCGCGGCCTGCTG 1191

Mtb 1807 GCACCGGGCTGCTTCATCCCTGTGGCCGAATCCATCAACCTTGCAGGCGAATTGGATAGA 1866 | || | || ||||| |||||||||| |||||||| |||| ||| ||| |

M.smeg 1192 TCGCCCGACTCGTTCATCGGCGTGGCCGAATCGATCAACCTCGCAGGGGAACTGGGCCGG 1251

Mtb 1867 TGgGTGCTGCGgAGgGCCTGCAATGAATTCTCCGAGTGGCAGTCAGCCGGTTTGGGCCAC 1926 ||||||||||| | ||| ||| || |||||| ||||| | | ||| |||||| |

M.smeg 1252 TGGGTGCTGCGCACGGCATGCGCCGAGTTCTCCCGGTGGCGGGCCAACGGCGTGGGCCGC 1311

Mtb 1927 GACG-CGCTGCTGCGTATCAACGTCTCAGCTGGACAGCTGGTGACGGgCGgGTTTGTTGA 1985

M.smeg 1312 AACATCG-TCTTGCGGATCAACGTCTCACCGGTCCAGCTCGTGACCGACGGATTCGTCGA 1370

Mtb 1986 CTTCGTCGCAGACACGATCGgCCAGCACGGTCTGGACGC-CTCGTCCGTGTGTTTGGAAA $2 \odot 44$

M.smeg 1371 GTCGGTGGCGGGCATCATGAAAGAGTTCGGTCTGC-CGCGCGGGTCGGTGTGTCTGGAGA 1429 


\footnotetext{
Mtb 2045 TCACCGAAACGTTGTGgTGCAAGACCTACATACCGCCAGAGCCACCCTGGCTCGACT-C 2103

M.smeg 1430 TCACCGAAAGCGTTGTGGTGCAGGACATCGAGACCACCCGCACCACGCTGACGGGTCTGC 1489

Mtb 2104 AAAGAGTCGgCGTTCACATCGCTATCGACGATTTCGGCACCGGCTATAGCGCCATATCA 2163 | | | ||||||| || | || ||||||||||||||||||| || ||| | ||

M.smeg 1490 ACA-ACGTCGGCGTCCAGGTGGCCATCGACGATTTCGGCACCGGTTACAGCGTGTTGTCG 1548

Mtb 2164 CTgTTGCAGACGCTACCGATCGACACGCTCAAGATCGACAAAACATTCGTGCGGC-AACT 2222

M.smeg 1549 CTGCTGAAATCACTGCCGGTGGACACGCTGAAGATCGACCGCAGCTTCGTG-GCCGAACT $16 \odot 7$

Mtb 2223 CGgAACCAACACTAGCGATCTGGTCATTGTGCGCGGCATCATGACACTCGCCGAAGGCTT 2282

M.smeg 1608 GGGCTCCAACCCGGGCGACCTTCCGATCGTGCGGGCCGTGATCGCGCTGGCGGGGGCCTT 1667

Mtb 2283 cCAACTCGATGTAGTAGCCGAAGgCGTCGAGACCGAG-GCTGCCGCCAGAATTCTATTGG 2341

M.smeg 1668 CGGACTACAACTGGTGGCCGAGGGCGTGGAGACCGAGCGC-GCCGCGCTCACCCTGCTGC 1726

Mtb 2342 ATCAGCGCTGTTACCGTGCGCAAGGCTTCTTGTTCTCCCGGCCTGTCCCCGGGGAG-GCC 2400

M.smeg 1727 GCCACGGGTGTTACCGCGCGCAGGGGTTCCTGCTGTCCAAACCCATCCT-GGGCAGTGAG 1785

Mtb 2401 ATGCGg-CACATGTTGTCCGCACGACGACTACCGCCGACCTGCATACCTGCAACT 2454

M.smeg 1786 ATGCAGACCCTTCTTG-CCAAAGGCCGTGTGCCGGTGCACTTCTCAGCTGCGCCT 1839
} 


\subsection{Mb1389c and MSMEG_2196 gene and protein sequences}

\subsubsection{Mb1389c gene sequence}

ATGTGCAACGACACCGCGACGCCGCAGCTTGAGGAGCTCGTCACCACCGTAGCCAACCAG CTCATGACAGTCGACGCTGCCACGTCAGCCGAAGTCAGTCAGCGCGTTTTGGCCTATCTA GTGGAACAGCTGGGCGTAGATGTCAGCTTTTTGCGTCATAACGATCGCGACAGGCGCGCG ACGAGGCTGGTGGCCGAATGGCCACCTCGCCTCAACATACCGGACCCCGATCCGCTCAGG CTGATCTACTTCGCTGATGCCGACCCGGTGTTTGCGCTATGCGAACACGCCAAAGAGCCT CTCGTGTTCCGGCCCGAGCCGGCCACCGAGGACTATCAACGCCTCATCGAAGAAGCCCGC GGGGTTCCGGTAACGTCGGCTGCCGCCGTGCCGCTGGTATCTGGCGAGATCACCACTGGA CTGCTGGGGTTCATCAAGTTCGGTGATCGGAAATGGCACGAGGCCGAGCTTAACGCCCTC ATGACCATCGCTACACTCTTCGCCCAGGTGCAGGCTCGCGTCGCCGCCGAGGCGCGGCTT CGCTATCTGGCCGACCATGACGATCTGACCGGACTGCATAACCGTCGCGCGTTGCTGCAG CACCTGGACCAAAGACTGGCCCCCGGACAACCTGGCCCGGTCGCGGCGCTATTTCTCGAC TTGGACCGCCTCAAGGCCATCAACGACTACCTGGGCCACGCCGCCGGTGACCAGTTCATC CATGTGTTCGCCCAACGGATCGGTGACGCACTCGTTGGCGAGAGCCTGATCGCCCGACTC GGCGGCGACGAATTCGTCCTCATACCCGCATCTCCAATGAGTGCCGATGCCGCTCAACCG CTCGCCGAACGTCTTCGCGACCAGCTCAAGGACCACGTCGCTATCGGCGGTGAGGTGCTC ACCCGCACCGTCAGTATCGGTGTCGCCTCAGGGACTCCCGGACAGCACACACCGTCGGAC CTCCTGCGCCGAGCCGACCAAGCCGCTCTGGCAGCCAAACACGCCGGCGGAGATAGCGTC GCGATTTTCACCGCGGACATGTCGGTCAGCGGCGAACTGCGCAACGATATTGAACTACAC CTTCGACGTGGTATCGAATCCGACGCCCTTCGCCTGGTCTACCTACCCGAGGTCGACCTA CGGACCGGCGACATTGTCGGGACCGAGGCATTGGTCCGGTGGCAGCACCCCACCCGTGGG CTGCTGGCACCGGGCTGCTTCATCCCTGTGGCCGAATCCATCAACCTTGCAGGCGAATTG GATAGATGGGTGCTGCGGAGGGCCTGCAATGAATTCTCCGAGTGGCAGTCAGCCGGTTTG GGCCACGACGCGCTGCTGCGTATCAACGTCTCAGCTGGACAGCTGGTGACGGGCGGGTTT GTTGACTTCGTCGCAGACACGATCGGCCAGCACGGTCTGGACGCCTCGTCCGTGTGTTTG GAAATCACCGAAAACGTTGTGGTGCAAGACCTACATACCGCCAGAGCCACCCTGGCTCGA CTCAAAGAAGTCGGCGTTCACATCGCTATCGACGATTTCGGCACCGGCTATAGCGCCATA TCACTGTTGCAGACGCTACCGATCGACACGCTCAAGATCGACAAAACATTCGTGCGGCAA CTCGGAACCAACACTAGCGATCTGGTCATTGTGCGCGGCATCATGACACTCGCCGAAGGC TTCCAACTCGATGTAGTAGCCGAAGGCGTCGAGACCGAGGCTGCCGCCAGAATTCTATTG GATCAGCGCTGTTACCGTGCGCAAGGCTTCTTGTTCTCCCGGCCTGTCCCCGGGGAGGCC ATGCGGCACATGTTGTCCGCACGACGACTACCGCCGACCTGCATACCTGCAACTGACCCG GCGTTATCTTG .

\subsubsection{Mb1389c protein sequence}

MCNDTATPQLEELVTTVANQLMTVDAATSAEVSQRVLAYLVEQLGVDVSFLRHNDRDRRA TRLVAEWPPRLNIPDPDPLRLIYFADADPVFALCEHAKEPLVFRPEPATEDYQRLIEEAR GVPVTSAAAVPLVSGEITTGLLGFIKFGDRKWHEAELNALMTIATLFAQVQARVAAEARL RYLADHDDLTGLHNRRALLQHLDQRLAPGQPGPVAALFLDLDRLKAINDYLGHAAGDQFI HVFAQRIGDALVGESLIARLGGDEFVLIPASPMSADAAQPLAERLRDQLKDHVAIGGEVL TRTVSIGVASGTPGQHTPSDLLRRADQAALAAKHAGGDSVAIFTADMSVSGELRNDIELH LRRGIESDALRLVYLPEVDLRTGDIVGTEALVRWQHPTRGLLAPGCFIPVAESINLAGEL DRWVLRRACNEFSEWQSAGLGHDALLRINVSAGQLVTGGFVDFVADTIGQHGLDASSVCL EITENVVVQDLHTARATLARLKEVGVHIAIDDFGTGYSAISLLQTLPIDTLKIDKTFVRQ LGTNTSDLVIVRGIMTLAEGFQLDVVAEGVETEAAARILLDQRCYRAQGFLFSRPVPGEA MRHMLSARRLPPTCIPATDPALS. 


\subsubsection{MSMEG_2196 gene sequence}

GTGTCCGAGAGCCTGGACGTGCTGGTCACGTCCGTCGCCACTCAGCTCATGGCAGTCGAG GCGGCCACCTCGGTGTCGGTCAGCCAGCAGGTGCTCGCCGAACTGGTGTCGTTCTTCGAT GTCGATGTGAGCTTCCTGCGCCACAACGACCACCGCGCCCACGCCACGAGGCTCGTCGCC GAGTGGCCGCCGCGTCCGGTCGACGCCGCCACCGACCCGATCGCCGTCGTCCACTTCGCC GATGCCGACCCGGTGTTCGCCATGGCCGAACACCTCAAGGAACCCGCGGTGTTCCGGCCC GAACCGCTCTACGACGACTATCAGCGCACCATCGAGGCAGGCCGGCACATCCCCGCCACG TCGATGGCGTGCGTCCCGCTGCTCAGCGGCGACGTCACGACCGGTGTGCTGGGCTTCGTC AAGTTCGGCGACCGCGAGTGGCTGCCCGCAGAACTCAACGCGCTCAAGGCGATCGCGTCG TTGTTCGCGCAGGTGCAGGCCCGGATCGAGGCCGAGGAACGCCTGCGTTACCTGGCCGAC CACGATCACCTGACCGGCCTGTACAACCGTCGCGCCCTGATGGCGCACCTGGAAGCGAGA CTCGCTCCGGGACAACCCGGTCCGGTCGCGGTGATGTTCTTCGACCTGGACCGGCTGAAG GCGATCAACGACTACCTGGGTCACACCGCGGGTGACGCGTTCATCAGCATCCTCGCGCAG CGACTGCAACGCGGTGACGACGCTCCGAAGCTGATAGCGCGCCTCGGCGGCGACGAGTTC GTCGTCGTCCCCGACGATCCCATGTCGCTGGATGAGGCCACCGCGCTGGCCTACCGGCTG CAGTCGGTGCTGCGCGAACGCGTCACCGTCGACGGTGAGATGCTCACTCGAACCGTGAGC ATCGGTATCGCACAAGGCATTCCGGGTAAGGATTCAACGTCCGACGTGCTCAACCGGGCC GACCACGCGGTATTGACGGCCAAGGGTTCCGGCGGCAACCGCGTCGCGGTCTTCTCCGAC GCGATGGCCATGGAGATCGACTTCCGCAACGACATCGAACTGCACCTGCAGAGCGTGATC GAAGGCGGCGCACTGGTGCTGCACTACCTGCCGGAAATCGACATGCGCACCGGTGAGGTG CTGGCGGCAGAAGCGCTGGTCCGCTGGGAGCATCCCACCCGCGGCCTGCTGTCGCCCGAC TCGTTCATCGGCGTGGCCGAATCGATCAACCTCGCAGGGGAACTGGGCCGGTGGGTGCTG CGCACGGCATGCGCCGAGTTCTCCCGGTGGCGGGCCAACGGCGTGGGCCGCAACATCGTC TTGCGGATCAACGTCTCACCGGTCCAGCTCGTGACCGACGGATTCGTCGAGTCGGTGGCG GGCATCATGAAAGAGTTCGGTCTGCCGCGCGGGTCGGTGTGTCTGGAGATCACCGAAAGC GTTGTGGTGCAGGACATCGAGACCACCCGCACCACGCTGACGGGTCTGCACAACGTCGGC GTCCAGGTGGCCATCGACGATTTCGGCACCGGTTACAGCGTGTTGTCGCTGCTGAAATCA CTGCCGGTGGACACGCTGAAGATCGACCGCAGCTTCGTGGCCGAACTGGGCTCCAACCCG GGCGACCTTCCGATCGTGCGGGCCGTGATCGCGCTGGCGGGGGCCTTCGGACTACAACTG GTGGCCGAGGGCGTGGAGACCGAGCGCGCCGCGCTCACCCTGCTGCGCCACGGGTGTTAC CGCGCGCAGGGGTTCCTGCTGTCCAAACCCATCCTGGGCAGTGAGATGCAGACCCTTCTT GCCAAAGGCCGTGTGCCGGTGCACTTCTCAGCTGCGCCTCGCATGTGA .

\subsubsection{MSMEG_2196 protein sequence}

MSESLDVLVTSVATQLMAVEAATSVSVSQQVLAELVSFFDVDVSFLRHNDHRAHATRLVA EWPPRPVDAATDPIAVVHFADADPVFAMAEHLKEPAVFRPEPLYDDYQRTIEAGRHIPAT SMACVPLLSGDVTTGVLGFVKFGDREWLPAELNALKAIASLFAQVQARIEAEERLRYLAD HDHLTGLYNRRALMAHLEARLAPGQPGPVAVMFFDLDRLKAINDYLGHTAGDAFISILAQ RLQRGDDAPKLIARLGGDEFVVVPDDPMSLDEATALAYRLQSVLRERVTVDGEMLTRTVS IGIAQGIPGKDSTSDVLNRADHAVLTAKGSGGNRVAVFSDAMAMEIDFRNDIELHLQSVI EGGALVLHYLPEIDMRTGEVLAAEALVRWEHPTRGLLSPDSFIGVAESINLAGELGRWVL RTACAEFSRWRANGVGRNIVLRINVSPVQLVTDGFVESVAGIMKEFGLPRGSVCLEITES VVVQDIETTRTTLTGLHNVGVQVAIDDFGTGYSVLSLLKSLPVDTLKIDRSFVAELGSNP GDLPIVRAVIALAGAFGLQLVAEGVETERAALTLLRHGCYRAQGFLLSKPILGSEMQTLL AKGRVPVHFSAAPRM . 


\section{References}

1. Akhter, Y.; Tundup, S.; Hasnain, S. E. 2007. Novel biochemical properties of a CRP/FNR family transcription factor for Mycobacterium tuberculosis. International Journal of Medical Microbiology. 297:451-457.

2. Aldrich, P.; Paul, R.; Goymer, P.; Rainey, P.; Jenal, U. 2003. Role of GGDEF regulator PleD in polar development of Calcubacter crescentus. Molecular Microbiology. 47:1695-708.

3. Aral, B; Kamoun, P. 1997. The proline biosynthesis in living organisms. Amino Acids. 13:189-217.

4. Arcondeguy, T.; Jack, R.; Merrick, M. 2001. $\underline{\underline{I I I}}_{\text {signal transduction proteins, }}$ pivotal players in microbial nitrogen control. Microbiology Molecular Biology. 65:80-105.

5. Arico, B.; Miller, J. F.; Roy, C.; Stibitz, S.; Monack, D. et al. 1989. $\underline{\text { Sequences }}$ required for expression of Bordetella pertussis virulence factors share homology with prokaryotic signal transduction proteins. Proceedings of the National Academy of Sciences USA. 86:6671-75.

6. Arvind, L.; Ponting, C. P. 1997. The GAF domain: an evolutionary link between diverse phototransduction protiens. Trends Biochem. Sci. 22: 458-459.

7. Avinash, R.; Visweswariah, S. S. 2006. New messages from old messengers: cAMP and mycobacteria. TRENDS in Microbiology. 14(12):544-550.

8. Bai, G.; McCues, L. A.; McDonough, K. A. 2005. Charaterization of Mycobacterium tuberculosis Rv3676 (CRP ${ }_{\mathrm{Mt}}$ ), a cyclic AMP receptor proteinlike DNA binding protein. Journal of Bacteriology. 187(22):7795-7804. 
9. Baker, D. A.; and Kelly, M. 2004. Structure, function and evolution of microbial adenylyl and guanylyl cyclases. Molecular Microbiology. 52:12291242.

10. Bayer, A. S.; Coulter, S. N.; Stover, C. K.; Schwan, W. R. 1999. Impact of the high-affinity proline permease gene (putP) on the virulence of Staphylococcus aureus in experimental endocariditis. Infect. Immun. 67:740-744.

11. Benziman, M.; Haigler, C. H.; Brown, R. M.; White, A. R.; Cooper, K. M. 1980. Cellulose biogenesis: polymerization and crystallization are coupled processes in Acetobacter xylinum. Proc. Natl. Sci. USA. 77:6678-6682.

12. Beste, D. J. V.; Laing, E.; Bonde, B.; Avignone-Rosa, C.; Bushell, M. E.; McFadden, J. J. 2007. Transcriptomic analysis identifies growth rate modulation as a component of the adaptation of mycobacteria to survival inside the macrophages. Journal of Bacteriology. 189(11):3969-3976.

13. Betts, J. c.; Lukey, P.T.; Robb, L. C.; McAdam, R. A.; Duncan, K. 2002. Evaluation of a nutrient starvation model of Mycobacterium tuberculosis persistence by gene and protein expression profiling. Molecular Microbiology. 43(3):717-731.

14. Blokpoel, C. J.; Murphy, H. N.; O’Toole, R.; Wiles, S.; Runn, S. C.; Stewart, G. R.; Young, D. R.; Robertson, B. D. 2005. Tetracycline-inducible gene regulation in mycobacteria. Nucleic Acid Research. 33(2) e22.

15. Bobrov, A. G.; Kirillina, O.; Perry, R. D. 2005. The phosphodiesterase activity of the HmsP EAL domain is required for negative regulation of biofilm formation in Yersinia pestis. FEMS Microbiology Letters. 247:123-30. 
16. Boles, B. R.; McCarter, L. L. 2002. Vibrio parahaemolyticus scrABC, a novel operon affecting swarming and capsular polysaccharide regulation. Journal of Bacteriology. 185:5946-54.

17. Bomchil, N.; Watnick, P.; Kolter, R. 2003. Identification and characterization of a Vibrio cholerae gene, $m b a A$, involved in maintenance of biofilm architecture. Journal of Bacteriology. 185:1384-90.

18. Broeze, R. J.; Solomon, C. J.; Pope, D. H. 1978. Effects of low temperature on $\underline{\text { in vivo and in vitro protein synthesis in Escherichia coli and Pseudomonas }}$ fluorescens. J. Bacteriol. 134:861-874.

19. Chang, A. L.; Tuckerman, J. R.; Gonzalez, G.; Mayer, R.; Weinhouse, H. et al. 2001. Phosphodiesterase A1, a regulator of cellulose synthesis in Acetobacter Xylinum, is a heme-based sensor. Biochemistry. 40:3420-26.

20. Charbonneau, H.; Prusti, R. K.; LeTrong, H.; Sonnenburg, W. K. et al. 1990. Identification of a noncatalytic c-GMP binding domain conserved in both the cGMP-stimulated and photoreceptor cyclic nucleotide phosphodiesterases. Proceeding of National Academic Sciences. USA 87, 288-292.

21. Checa, S. K.; Viale, A. M. 1997. The 70-KDa heat-shock protein/DnaK chaperon system is required for the productive folding of ribulose-biphosphate carboxylase subunits in Escheichia coli. Eur. J. Biochem. 248:848-855.

22. Chen, J. M.; German, G. J.; Alexander, D. C.; Ren, H.; Tan, T.; Liu, J. 2006. $\underline{\text { Roles of Lsr2 in colony morphology and biofilm formation of Mycobacterium }}$ smegmatis. J. Bacteriol. 188:633-641. 
23. Choi, K.; Schweizer, H. P. 2005. An improved method for rapid generation of unmarked Pseudomonas aeruginosa deletion mutants. BMC Microbiology. 5:30.

24. Choy, W. K.; Zhou, L.; Syn, C. K.; Zhang, L. H.; Swarup, S. 2004. MorA defines a new class of regulators affecting flagellar development and biofilm formation in diverse pseudomonas species. Journal of Bacteriology. 186:722128

25. Christen, M.; Christen, B.; Folcher, M.; Schauerte, A.; Jenal, U. 2005. Identification and characterization of a cyclic di-GMP-specific phosphodiesterase and its allosteric control by GTP. Journal of Biological Chemistry. 280:30829-30837.

26. Christen, B.; Christen, M.; Paul, R.; Schmid, F.; Folcher, M.; et al. 2006. Allosteric control of cyclic di-GMP signalling. Journal of Biology Chemistry. 281(42):32015-32024.

27. Cole, S. T.; Brosch, R.; Parkhill, J.; Garnier, T.; Churcher, C.; Harris, D.; Gordon, C. V; Eiglmeier, K.; Gas, S.; et al. 1998. Deciphering the biology of Mycobacterium tuberculosis from the complete genome sequence. Nature. 393:537-554.

28. Confer, D. L.; Eaton, J. W. 1982. Phagocyte impotence caused by an invasive bacterial adenylate cyclase. Science. 217:948-950.

29. Craig, E. A. 1985. The heat shock response. CRC Crit. Rev. Biochem. 18:239-280.

30. Csonka, L. N. 1981. Proline over-production results in enhanced osmotolerance in Salmonella typhimurium. Molecular gene Genetics. 182:8286. 
31. Culham, D. E.; Dalgado, C.; Gyles, C. L.; Mamelka, D.; MacLellan, S.; Wood, J. M. 1998. Osmoregulatory transporter ProP influences colonization of the urinary tract by Escherichia coli. Microbiology. 144:91-102.

32. D'Arggenio, D. A. and Miller, S. I. 2004. Cyclic di-GMP as a bacterial second messenger. Microbiology. 150:2497-2502.

33. De Mendoza, D.; Cronan Jr, J. E. 1983. Thermal regulation of membrane lipid fluidity in bacteria. Trends Biochem. Sci. 8:49-52.

34. Dhandayuthapani, S. 2007. Stress response of genes encoding putative stress signaling molecules of Mycobacterium tuberculosis. Frontiers in Bioscience. 1(12):4676-81.

35. Diamant, S.; Goloubinoff, P. 1998. Temperature-controlled activity of DnaKDnaJ-GrpE chaperons: a protein-folding arrest and recovery during and after heat shock depends on the substrate protein and the GrpE concentration. Biocheistry. 37:9688-9694.

36. Drenkard, E.; Ausubel, F. M. 2002. Pseudomonas biofilm formation and antibiotic resistance are linked to phenotypic variation. Nature. 416:740-43.

37. Enos-Berlage, J. L.; Guvenner, Z. T.; Keenan, C. E.; McCarter, L. L. 2005. Genetic determinants of biofilm development of opaque and translucent Vibrio Parahaemolyticus. Molecular Microbiology. 55:1160-82.

38. Feng, Y.; Huang, H.; Liao, J.; Cohen, S. N. 2001. Escherichia coli poly(A)binding proteins that interact with components of degradosomes or impede RNA decay mediated by polynucleotide phosphorylates and RNase. E. J. Biol. Chem. 276:31651-31656.

39. Friedman, H.; Lu, P.; Rich, A. 1971. Temperature control of initiation of protein synthesis in Escherichia coli. J. Mol. Biol. 61:105-121. 
40. Fujita, T.; maggio, A.; Garcia-Rios, M.; Stauffacher, C; Bressan, R. A.; Csonka, L. N. 2003. Identification of regions of the tomato $\gamma$-glutamyl kinase that are involved in allosteric regulation by proline. Journal of Biol. Chem. 278:1420314210.

41. Galperin, M. Y.; Nikolskaya, A. N.; and Koonin, E. V. 2001. Novel domains of the prokaryotic two-component signal transduction system. FEMS Microbiology Letters 203:11-21.

42. Garcia, B.; Latasa, C.; Sikabi, C.; Garcia-del Portillo,F.; Gamazo, C.; Lasa, I. 2004. Role of the GGDEF protein family in salmonella biosynthesis and biofilm formation. Molecular Microbioloy. 54:264-77.

43. Gosset, G.; Zhang, Z. G.; Nayyar, S. N.; Cuevas, W. A.; Saier, M. H. 2004. Transcriptome analysis of Crp-dependent catabolite control of gene expression in Escherichia coli. Journal of Bacteriology. 186:3516-3524.

44. Goymer, P.; Khan, S.; Malone, J.; Gehrig, S.; Spiers, A.; Rainey, P. 2006. Adaptive divergence in experimental populations of Pseudomona fluorescens. II. The role of the GGDEF regulator WspR in evolution and development of the wrinkly spreader phenotype. Genetics. 173:515-26.

45. Graumann, P.; Wendrich, T. M.; Webber, M. H.; Schroder, K.; Marahiel, M. A. 1997. A family of cold shock proteins in Bacillus subtillis is essential for cellular growth and for efficient protein synthesis at optimal and low temperatures. Mol. Microbiol. 25:741-756.

46. Green, J.; Scott, C.; Guest, J. 2001. Functional versatility in the CRP-FNR superfamily of transcription factors: FNR and FLP. Adv. Microb. Physiol. 44:1-34. 
47. Gupta, S.; Chatterji, D. 2005. Stress responses in mycobacteria. IUBMB Life 57:149-150.

48. Hall-Stoodley, L.; Lappin-Scott, H. 1998. Biofilm formation by the rapidly growing mycobacterial species Mycobacterium fortuitum. FEMS Microbiol. Lett. 168:77-84.

49. Hall-Stoodley, L.; Costerton, J. W.; Stoodley, P. 2004. Bacterial biofilms: from the natural environment to infectious diseases. Nature Reviews Microbiology. 6:546-51.

50. Hall-Stoodley, L.; Brun, O. S.; Ploshyan, G.; Barker, L. P. 2006. Mycobacterium marinum biofilm formation reveals cording morphology. FEMS Microbiol. Lett. 257:43-49.

51. Harth, G.; Maslesa-Galic, S.; Tullius, M. V.; Horwitz, M. A. 2005. All four Mycobacterium tuberculosis glnA genes encode glutamine synthetase activities but only GlnA1 is abundantly expressed and essential for bacterial homeostasis. Molecular Microbiology. 58(4):1157-1172.

52. Hartl, F. U. 1996. Molecular chaperones in cellular protein folding. Nature. 381:571-579.

53. Hecht, G. B.; Newton, A. 1995 Identification of a Novel Response Regulator required for the Swarmer-to-Stalked-Cell transition in caulobacter. Journal of Bacteriology. 177: 6223-6229. 
54. Hickman, J. W.; Tifera, DF.; Harwood, CS. 2005. A chemosensory system that regulates biofilm formation through modulation of cyclic diguanylate levels. Proc. Natl. Acad. Sci. USA. 102:14422-27.

55. Hisert, K. B.; MacCoss, M.; Shiloh, M. U.; Darwin, K. H.; Singh, S.; Jones, R. A.; Ehrt, S.; Zhang, Z.; Gaffney, B. L.; Gandorta, S.; Holden, D. W.; Murray, D.; Nathan, C. 2005. A glutamate-alanine-leucine (EAL) domain protein of $\underline{\text { salmonella controls bacterial survival in mice, antioxidant defence and killing of }}$ macrophages: role of cyclic diGMP. Molecular Microbiology. 56:1234-1245.

56. Ho, S. N.; Hunt, H. D.; Horton, R. M.; Pullen, J. K.; Pease, L. R. 1989. Sitedirected mutagenesis by overlap extension using the polymerase chain reaction. Gene. 77:51-59.

57. Hoffman, L. R.; D’Argenio, D. A.; MacCoss, M. J.; Jones, R. A.; Miller, S. I. 2005. Aminoglycoside antibiotics induce bacterial biofilm formation. Nature. 436:1171-75.

58. Hu, Y. M.; Butcher, P. D.; Sole, K.; Mitchison, D. A.; Coates, A. R. 1998. Protein synthesis is shutdown in dormant Mycobacterium tuberculosis and is reversed by oxygen or heat-shock. FEMS Microbiol. Lett. 158:139-145.

59. Hubbard, T. J; Sander, C. 1991. The role of heat-shock and chaperone proteins in protein folding: possible molecular mechanisms. Protein Eng. 4:711-717.

60. Jaggi, R.; Van Heeswijk, W. C.; Westerhoff, H. V.; Ollis, D. L.; Vasudevan, S. G. 1997. The two opposing activities of adenlyltransferase reside in distinct homologous domains with intramolecular signal transduction. EMBO J. 16:5562-5571. 
61. Javelle, A.; Merrick, M. 2005. Complex formation between AmtB and GlnK: an ancestral role in prokaryotic nitrogen control. Biochem Soc Trans. 33:170172.

62. Jenal, U. 2004. Cyclic di-guanosine-monophosphate comes of age: a novel secondary messenger involved in modulating cell surface structures in bacteria. Current Opinion in Microbiology 7: 185-191.

63. Jiang, P.; Peliska, J. A.; Ninfa, A. J. 1998. Enzymological characterization of the signal-transduction uridylyltransferase/uridylyl-removing enzyme (EC 2.7.7.59) of Escherichia coli and its interaction with the $\mathrm{P}_{\underline{I I}}$ protein. Biochemistry. 37:12782-12794.

64. Jiang, P.; Ninfa, A. J. 1999. Regulation of autophosphorylation of Escherichia coli nitrogen regulator II by the $\mathrm{P}_{\text {II }}$ signal transduction protein. Journal of Bacteriology. 181:1906-1911.

65. Joerger, R. D.; Jacobson, M. R.; Bishop, P. E. 1989. Two nifA genes required for expression of alternative nitrogenises by Azotobacter vinelandii. Journal of Bactriology. 171(6):3258-67.

66. Johnson, M. R.; Montero, C. I.; Conners, S. B.; Shockley, K. R.; Bridger, S. L.; Kelly, R. M. 2005. Population density-dependent regulation of exopolysaccharide formation in the hyperthermophilic bacterium Thermotoga maritima. Molecular Microbiology. 55:664-74.

67. Jones, P. G., VanBogelen, R. A. \& Neidhardt, F. C. 1987. Induction of proteins in response to low temperature in Escherichia coli. J. Bacteriol. 169:2092-2095.

68. Kader, A.; Simm, R.; Gerstel, U.; Morr, M.; Romling, U. 2006. Hierarchical involvement of various GGDEF domain proteins in rdar morphotype 
development of Salmonella enterica serovar Typhimurium. Molecular Microbiology. 60:602-16.

69. Kancher, T.; Schultz, A.; Schultz, J. E. 2002. A GAF-domain-regulated adenylyl cyclase from anabena is a self-activating cAMP switch. The EMBO Journal. 15;21(14):3672-80.

70. Karatan, E.; Duncan, T. R.; Watnick, P. I. 2005. NspS, a predicted polyamine sensor, mediates activation of Vibrio cholerae biofilm formation by norspermidine. Journal of Bacteriology. 187:7434-43.

71. Kaur, H.; Khuller, G. 1995. Role of cyclic adenosine monophosphate in phospholipid synthesis in Mycobacterium smegmatis ATCC 607. Lipids. 30: 345-349.

72. Kirillian, O.; Fetherston, JD; Bobrov, AV; Abney, J.; Perry, RD. 2004. HmsP, a putative phosphodiesterase, and HmsT, a putative diguanylate cyclise, control Hms-dependent biofilm formation in Yersinia pestis. Molecular Microbiology. 54:75-88.

73. Korner, H.; Sofia, H.J.; Zumft, W.G. 2003. Phylogeny of the bacterial superfamily of CRP-FNR transcription regulators: exploiting the metabolic spectrum by controlling alternative gene programs. FEMS Microbiol. Rev. 27:559-592.

74. Kovacikova, G.; Lin, W.; Skorupski, K. 2005. Dural regulation of genes involved in acetoin biosynthesis and motility/biofilm formation by the virulence activator AphA and the acetate-responsive LysR-type regulator AlsR in Vibrio cholerae. Mold. Microbiol. 57:420-433. 
75. Kulasekara, H.; ventre, I.; Kulasekara, B. R.; Lazunski, A.; Filloux, A.; Lory, S. 2005. A novel two-component system controls the expression of Pseudomonas aeruginosa fimbrial cup genes. Molecular Microbiology. 55:368-80.

76. Kulesekara, H.; Lee, V.; Brencic, A.; Liberati, N.; Urbach, J.; et al. 2006. Analysis of Pseudomonas aeruginosa diguanylate cyclise and phosphodiesterases reveals a role for bis-(3'-5')-cyclic-GMP in virulence. Proceeding of the National Academy of Sciences. USA. 103:2839-44.

77. Kutchma, S. L.; Connolly, J. P.; O’Toole, G. A. 2005. A three-component regulatory system regulates biofilm maturation and type III secretion in Pseudomonas aeruginosa. Journal of Bacteriology. 187:1441-54.

78. Lee, C. H. 1977. Identification of adenosine 3', 5'-monophosphate in Mycobacterium smegmatis. Journal of Bacteriology. 132:1031-1033

79. Leigh, J. A.; Dodsworth, J. A. 2007. Nitrogen regulation in bacteria and archae. Annu. Rev. Microbiol. 61:349-77.

80. Leisinger, T. 1996. Biosynthesis of proline in: Escherichia coli and salmonella. Cellular and Molecular Biology. (Neidhardt, F. C., Ed.). 434-441. ASM press, Washington, DC.

81. Levi, A.; Jenal, U. 2006. Holdfast formation in motile swarmer cells optimizes surface attachment during Caulobacter crescentus development. Journal of Bacteriology. 188:5315-18.

82. Lim, B.; Beyhan, S.; Meir, J.; Yildiz, F. H. 2006. Cyclic di-GMP signal transduction systems in Vibrio cholerae: modulation of rugosity and biofilm formation. Molecular Microbiology. 60:331-48. 
83. Lindquist, S.; Craig, E. A. 1988. The heat-shock proteins. Annu. Rev. Genet. 22:631-677.

84. Lowrie, D. B.; Aber, V. R.; Jackett, P. S. 1979. Phagosome-lysosome fusion and cyclic adenosine 3':5'-monophosphate in macrphages infected with Mycobacterium microti, Mycobacterium bovis BCG or Mycobacterium leparemurium. Journal of Genetic Microbiology. 110:431-441.

85. Mahswaran, M.; Forchhammer, K. 2003. Carbon-source-dependent nitrogen regulation in E. coli is mediated through glutamine-dependent GlnB signalling. Microbiology. 149:2163-2172.

86. Mao, X.; Huo, Y.; Buck, M.; Kolb, A.; Wang, Y. 2007. Interplay between

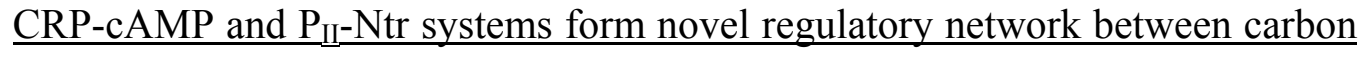
assimilation in Escherichia coli. Nucleic Acids Research. 35:1432-1440

87. Merkel, T. J.; Barros, C.; and Stibitz, S. 1998. Characterization of the bvgR locus of Bordetella pertusis. Journal of Bacteriology. 180:1682-1690.

88. Matrix Science, 2007. http://www.matrixscience.com/help/results_help.html .

89. Montgomery, B. L.; Lagarias, J. C. 2002. Phytochrome ancestry: sensors of bilins and light. Trends in Plant Science. 7(8):357-66.

90. Mukamolova, G.V.; Turapov, O. A.; Young, D. I.; Kaprelyants, A. S.; Kell, D. B.; Young, M. 2002a. A family of autocrine growth factors in Mycobacterium tuberculosis. Mol Microbiol 46: 623-635.

91. Murphy, D. J.; Brown, J. R. 2007. Identification of gene targets against dormant phase Mycobacterium tuberculosis infection. BMC Infectious Disease. 7(84):1-16. 


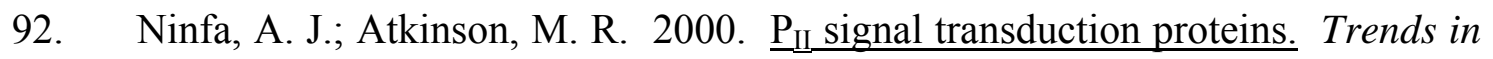
Microbiology. 8(4):172-9.

93. Ninfa, A. J.; Jiang, P. 2005. $\underline{\text { III }}_{\text {signal transduction proteins: sensors of alpha- }}$ ketoglutarate that regulate nitrogen metabolism. Current Opinion in Microbiology. 8:168-173.

94. Nyka, W. 1974. Studies on the effect of starvation on mycobacteria. Infect. Immun. 9: $843-850$.

95. Ojha, A. K., Mukherjee, T. K., and Chatterji, D. 2000. High intracellular level of guanosine tetraphosphate in Mycobacterium smegmatis changes the morphology of the bacterium. Infect. Immun. 68:4084 - 4091.

96. Padh, H.; Venkitasubramanian, T. A. 1976. Adenosine 3',5' monophosphate in Mycobacterium pheli and Mycobacterium tuberculosis H37Ra. Microbios 16:183-189.

97. Padh, H.; Venkitasubramanian, T. A. 1977. Adenosine 3',5' monophosphate in mycobacteria. Life Science. 20:1273-1280.

98. Parish, T.; Gordhan, B. G.; McAdam, R. A.; Duncan, K.; Mizrahi, V.; Stoker, N. G. 1999. Production of mutants in amino acid biosynthesis genes of Mycobacterium tuberculosis by homologous recombination. Microbiology. 145:3497-3503.

99. Parish, T.; Stoker, N. G. 2000. $g \ln E$ is an essential gene in Mycobacterium tuberculosis. Journal of Bacteriology. 182(20):5715-5720

100. Parsek, M. R.; Singh, P. K. 2003. Bacterial biofilms: an emerging link to disease pathogenesis. Annual Reviews Microbiology. 57:677-701. 
101. Paul, R.; Weiser, S.; Amiot, NC.; Chan, C.; Schirmer, T.; et al. 2004. Cell cycle-dependent dynamic localization of a bacterial response regulator with a novel di-guanylate cyclase output domain. Genes Development. 18:715-27.

102. Phadtare, S. 2004. Recent development in bacterial cold-shock response. Curr. Issues Mol. Biol. 6:125-136.

103. Rajagopal, S.; Key, J. M.; Purcell, E. B.; Boerema, D. J.; Moffat, K. 2004. Purification and initial characterization of a putative blue ligh-regulated phophodiesterase from Echerichia coli. Photochemistry and Photobiology. 80:542-47.

104. Read, R.; Pashley, C. A.; Smith, D.; Parish, T. 2007. The role of GlnD in ammonia assimilation in Mycobacterium tuberculosis. Tuberculosis (Edinburgh, Scotland). 87(4):384-390.

105. Reitzer, L. J.; Magasanik, B. 1986. Transcription of $g \ln A$ in E. coli is stimulated by activator bound to sites far from the promoter. Cell. 45:785-792.

106. Reitzer, L. 2003. Nitrogen assimilation and global regulation in Escherichia coli. Annu. Rev. Microbiology. 57:155-76.

107. Rhee, S.; Chock, P. B.; Stadtman, E. R. 1989. Regulation of Escherichia coli glutamine synthetase. Adv. Enzymol. Relat. Areas Mol. 62:37-92.

108. Rickman, L.; Scott, C.; Hunt, D. M., Hutchinson, T.; Whalan, R.; Hinds, J.; Colston, M. J.; Green, J.; Buxton, R. S. 2005. A member of the cAMP receptor protein family of transcription regulators in Mycobacterium tuberculosis is required for virulence in mice and controls transcription of the $r p f A$ gene coding for a resuscitation promoting factor. Molecular Microbiology. 56(5):12741286 
109. Römling, U.; Gomelsky, M.; and Galperin, M. Y. 2005. C-di-GMP: the dawning of a novel bacterial signalling system. Molecular Microbiology. 57(3): 926-639.

110. Römling, U.; and Amikam, D. 2006. Cyclic di-GMP as a second messenger. Curr. Opin. Microbiology. 9:218-228.

111. Ross, W. H.; Aloni, Y.; Michaeli, D.; Weinberger-Ohana, P.; Mayer, R.; Braun, S.; de Wroom, E.; van der Marel, G. A.; van Boom, J. H. et al., 1987. $\underline{\text { Regulation of cellulose synthesis in Acetobacter xylinum by cyclic diguanylic }}$ acid. Nature. 325:279-281.

112. Ryan, R. P.; Fouhy, Y.; Lucey, J. F.; Dow, M. 2006. Cyclci di-GMP signaling in bacteria: recent advances and new puzzles. Journal of Bacteriology. 188:8327-8334.

113. Ryan, R. P.; Fouhy, Y.; Lucy, J. F.; Crossman, L. C.; Spiro, S.; He, Y. -W.; Shang, L. -H.; Heeb, S.; Williams, P.; Dow, J. M. 2006. Cell-cell signalling in Xanthomonas campestris involves an HD-GYP domain protein that functions in cyclic di-GMP turnover. Proceeding of the National Academy of Sciences USA. 103:6712-6717.

114. Ryjenkov, D. A.; Tarunita, M.; Moskvino, O. V.; Gomelsky, M. 2005. Cyclic diguanylate is a ubiquitous signalling molecule in bacteria: insight into biochemistry of the GGDEF protein domain. Journal of Bacteriology. 187:1792-1798.

115. Sambrook, J.; Russell, D. W.; Irwin, N.; Janseen, K. A. 2001. Molecular Cloning- A Laboratory Manual. III Edition. Cold Spring Harbor Press, Cold Spring Harbor, New York.

116. Sardiwal, S.; Kendall, S, L.; Movahedzadeh, F.; Rison, S. C.; Stroker, N. G.; Djordjevic, S. 2005. A GAF domain in the hypoxia/No-inducible 
Mycobacterium tuberculosis DosS protein binds haem. Journal of Molecular Biology. 353:9292-936.

117. Sassetti, C. M.; Rubin, E. J. 2003. Genetic requirements for mycobacterial survival during infection. Proc. Natl. Acad. Sci. USA. 100(22):12989-12994.

118. Schmidt, A. J.; Ryjenkov, D. A.; Gomelsky, M. 2005. Ubiquitous protein domain EAL encodes cyclic diguanylate-specific phosphoduesterase: enzymatically active and inactive EAL domains. Journal of Bactiriology. 187:4774-4781.

119. Schwan, W. R.; Coulter, S. N.; Ng, E. Y. W.; Langhorne, M. H.; Ritchie, H. D.; Brody, L. L.; Westbrock, S.; Bayer, A. S.; Folger, K. R.; Stover, C. K. 1998. Identification and characterization of the PutP proline permease that contributes to in vivo survival of Staphylococus aureus in animal models. Infect. Immun. 66:567-572.

120. Shapiro, B. M.; Stadtman, E. R. 1968. 5'-Adenylyl-O-tyrosine: the novel phosphodiester residue of adenylylated glutamine synthetase from Escherichia coli. J. Biol. Chem. 243:3769-3771.

121. Sharrock, R. A.; Quail, P. H. 1989. Novel phytochrome sequences in Arabidopsis thalianai: structure, evolution, and differential expression of a plant regulatory photoreceptor family. Genes and Development. 3(11):1745-57.

122. Simm, R.; Morr, M.; Kadar, A.; Nimtz, M.; Romling, U. 2004. GGDEF and EAL domains inversely regulate cyclic di-GMP levels and transition from sessility to motility. Moleclar Microbiology. 53:1123-34.

123. Singh, V. K.; Utaida, S.; Jackson, L. S.; Jayaswal, R. K.; Wlkinson, B. J.; Chamberlain, N. R. 2007. Role of dnaK locus in tolerance of multiple stresses in Staphylococcus aureus. Microbiology. 153:3162-3173. 
124. Smith, L. T. 1985. Characterization of a $\gamma$-glutamyl kinase from Escherichia coli that confers proline overproduction and osmotic tolerance. Journal of Bacteriology. 164:1088-1093.

125. Smeulders, M. J.; Keer, J.; Speight, R. A.; Williams, H. D. 1999. Adoptation of Mycobacterium smegmatis to stationary phase. J. Bacteriol. 181:270-283.

126. Stadtman, E. R. 1990. Discovery of glutamine synthestase cascade. Methods Enzymol. 182:793-809.

127. Stewart, G. R.; Snewin, V. A.; Walzl, G.; Hussell, T.; Tormay, P.; O’Gaora, P.; Goyal, M.; Betts, J.; Brown, I. N.; Young, D. B. 2001. Overexpression of heatshock prtoeins reduces survival of Mycobacterium tuberculosis in the chronic phase of infection. Nature Med. 7(6):732-737.

128. Stock, A. M.; Robinson, V. L.; Goudreau, P. N. 2000. Two-component signal transduction. Annual review of Biochemsitry. 69:183-215.

129. Strizhov, N.; Abraham, E.; Okresz, L.; Blickling, S.; Zilberstein, A.; Schell, J.; Koncz, C.; Szabados, L. 1997. Differential expression of two P5CS gnes controlling proline accumulation during salt-stress requires $\mathrm{ABA}$ and is regulated by ABA1, ABI1 and AXR2 in arabidopsis. Plant J. 12:557-569.

130. Tal, R.; Wong, H. C.; Calhoon, R.; Amikam, D.; Weinhouse, H.; Feal, A. L.; Cohen, A.; Sapir, S.; Ohana, P.; Benziman, M. 1998. Three cdg operons control cellular turnover of cyclic di-GMP in Acetobacte rxylinum: genetic organization and occurrence of conserved domains in isoenzymes. J. Bacteriol. 180:4416-4425.

131. Tamayo, R.; Tischler, A. D.; Camilli, A. 2005. The EAL domain protein VieA is a cyclic diguanylate phosphodiesterase. Journal of Biological Chemistry. 280:33324-33330. 
132. Tamayo, R.; Pratt, J. T.; Camilli, A. 2007. Role of cyclic diguanylate in the regulation of bacterial pathogenesis. Annual Review of Microbiology. 61:131148.

133. The institute for genomic Research. http://www.tigr.org

134. Timm, J., Post, F. A., Bekker, L. G., Walther, G. B., et al. 2003. Differential expression of iron-, carbon-, and oxygen-responsive mycobacterial genes in the lungs of chronically infected mice and tuberculosis patients. Proc. Natl. Acad. Sci. USA 100: 14321 - 14326.

135. Tischler, A. D.; Lee, S. H.; Camilli, A. 2002. The Vibrio cholerae vieSAB locus encodes a pathway contributing to cholera toxin production. Journal of Bacteriology. 184:4104-13.

136. Tischler, A. D.; Camilli, A. 2004. Cyclic diguanylate (c-di-GMP) regulates Vibrio Cholerae biofilm formation. Molecular Microbiology. 53:8573-5882.

137. Tischler, A. D.; Camilli, A. 2005. Cyclic diguanylate regulate Vibrio Cholerae virulence gene expression. Infection Immunology. 73:5873-5882.

138. Tullius, M. V.; Harth, G.; Horwitz, M. A. 2003. Glutamine synthetase GlnA1 is essential for growth of Mycobacterium tuberculosis in human THP-1 Macrophages and guinea pigs. Infection and Immunity. 71(7):3927-3936.

139. Urao, T.; Yamaguchi-Shinozaki, K.; Shinozaki, K. 2001. Plant histidine kinases: an emerging picture of two-component signal transduction in hormone and environmental response. Science's Signal Transduction Knowledge Environment. (109):RE18.

140. Voskuil, M. I. 2004. Mycobacterium tuberculosis gene expression during environmental conditions associated with latency. Tuberculosis. 84:138-143. 
141. Voskuil, M. I.; Visconti, K. C.; Schoolnik, G. K. 2004. Mycobacterium tuberculosis gene expression during adaptation to stationary phase and lowoxygen dormancy. Tuberculosis. 84:218-227.

142. Wade, M.; Zhang, Y. 2004. Mechanisms of drug resistance in Mycobacterium tuberculosis. Front. Biosci. 9:975-94.

143. Walter, S.; Buchner, J. 2002. Molecular chaperones - cellular machines for protein folding. Angew. Chem. Int. Ed. Engl. 41:1098-1113.

144. Weber, M. H. W.; Marahiel, M. A. 2002. Cold shock response in Bacillus subtilis. Phil. Trans. R. Soc. Lond. B. 357:895-907.

145. Wilde, A.; Fiedler, B.; Borner, T. 2002. The cynobacterial phytochrome Cph2 inhibits phototaxis towards blue ligh. Molecular Microbiology. 44:981-88.

146. Wirth, J. J.; Kierszenbaum, F. 1982. Inhibitory action of elevated levels of adenosine- 3':5'-cyclic monophosphate on phagocytosis: effects on macrophages-Trypanosoma cruzi interaction. Journal of Immunology. 129:2759-2762.

147. World Health Organisation (WHO) Tuberculosis; 2008 Tuberculosis report. http://www.who.int/tb/publications/global_report/2008/en/index.html. http://www.who.int/tb/en/.

148. Zhang, Y.; Jacobs, W. R.; Vilcheze, C. 2004. Mchanisms of drug resistance in Mycobacterium tuberculosis. In pathogenesis of Tuberculosis. Washington, DC: ASM Press. $2^{\text {nd }}$ edition. 